\title{
HAMILTONIAN STABILITY OF THE GAUSS IMAGES OF HOMOGENEOUS ISOPARAMETRIC HYPERSURFACES II
}

\author{
Hui MA AND Yoshihiro OHNITA
}

(Received November 1, 2013, revised March 6, 2014)

\begin{abstract}
In this paper we determine the Hamiltonian stability of Gauss images, i.e., the images of the Gauss maps, of homogeneous isoparametric hypersurfaces of exceptional type with $g=6$ or 4 distinct principal curvatures in spheres. Combining it with our previous results in [12] and Part I [14], we determine the Hamiltonian stability for the Gauss images of all homogeneous isoparametric hypersurfaces. In addition, we discuss the exceptional Riemannian symmetric space $\left(E_{6}, U(1) \cdot \operatorname{Spin}(10)\right)$ and the corresponding Gauss image, which have their own interest from the viewpoint of symmetric space theory.
\end{abstract}

1. Introduction. This paper is a continuation of Part I ([14]). The image of the Gauss map, i.e., the so-called Gauss image, of any compact oriented isoparametric hypersurface in the standard unit sphere $S^{n+1}(1)$ is a compact minimal Lagrangian submanifold embedded in the complex hyperquadric $Q_{n}(\mathbf{C})$. This provides a nice class of compact Lagrangian submanifolds embedded in complex hyperquadrics. For example, the Gauss image of a compact oriented isoparametric hypersurface with $g$ distinct constant principal curvatures in $S^{n+1}(1)$ is orientable if and only if $2 n / g$ is even ([19]). Moreover, it is a compact monotone and cyclic embedded Lagrangian submanifold with minimal Maslov number $2 n / g=m_{1}+m_{2}$ ([14]). Here $m_{i}$ is the multiplicity of the $i$-th distinct principal curvature which satisfies $m_{i}=m_{i+2}$ for $i(\bmod g)$. Thus inspired by Y. G. Oh's work ([18]), it is an interesting problem to investigate the Hamiltonian stability of those compact minimal Lagrangian embedded submanifolds in $Q_{n}(\mathbf{C})$ obtained as the Gauss images of isoparametric hypersurfaces in $S^{n+1}(1)$.

It is well-known that all homogeneous isoparametric hypersurfaces in the standard unit sphere are obtained as principal orbits of the isotropy representation of rank 2 Riemannian symmetric pair $(U, K)$ ([9] , [22]). We have determined the Hamiltonian stability of Gauss images of homogeneous isoparametric hypersurfaces of classical type with $g=1,2,3$ ([12]) and $g=4$ distinct principal curvatures ([14]). In this paper we study the remaining cases for Gauss images of homogeneous isoparametric hypersurfaces of exceptional type

2010 Mathematics Subject Classification. Primary 53C42; Secondary 53C40, 53D12.

Key words and phrases. Lagrangian submanifold, minimal submanifold, Hamiltonian stability, Gauss map, isoparametric hypersurface.

The first named author is partially supported by NSFC grant No. 10971111, No. 11271213 and a scholarship from the China Scholarship Council. The second named author is partially supported by JSPS Grant-in-Aid for Scientific Research (A) No. 17204006, (S) No. 23224002, (C) No. 24540090, and the Priority Research of Osaka City University "Mathematics of knots and wide-angle evolutions to scientific objects". 
with $g=6$ or 4 distinct principal curvatures. Our method is based on harmonic analysis over compact homogeneous spaces and fibrations on homogeneous isoparametric hypersurfaces. In particular, we prove the following theorem in the case when $(U, K)$ is an exceptional symmetric space of rank 2, which was announced and proved for classical symmetric spaces $(U, K)$ of rank 2 in Part I ([14]).

THEOREM 1.1. Suppose that $(U, K)$ is not of type EIII, that is, $(U, K) \neq\left(E_{6}, U(1)\right.$. $\operatorname{Spin}(10))$. Then the Gauss image $L=\mathcal{G}(N)$ is not Hamiltonian stable if and only if $m_{2}-$ $m_{1} \geq 3$. Moreover if $(U, K)$ is of type EIII, then $\left(m_{1}, m_{2}\right)=(6,9)$ but $L=\mathcal{G}(N)$ is strictly Hamiltonian stable.

General discussion on our method was described in [14]. Sections 1, 2 and 3 of [14] are especially prerequisite for what follows. We continue to use the notation and the general introduction of Part I. This paper is organized as follows: For the convenience of readers, we review the most necessary background and formulations briefly in Section 2. In Sections 3 and 4, we determine the strict Hamiltonian stability of the Gauss images of compact homogeneous isoparametric hypersurfaces with $g=6$. In Section 5, we discuss the exceptional Riemannian symmetric space $\left(E_{6}, U(1) \cdot \operatorname{Spin}(10)\right)$ and determine the strict Hamiltonian stability of the corresponding Gauss image, which may have their own interest from the viewpoint of symmetric space theory.

Acknowledgments. The main results were already announced in [13]. This work was done during the first named author stayed at Osaka City University Advanced Mathematical Institute (OCAMI) in 2008-2009 and the second named author visited to Tsinghua University in Beijing. The authors are grateful to both institutes for their generous support and hospitality. They also would like to thank Professors Quo-Shin Chi, Josef Dorfmeister, Reiko Miyaoka and Zizhou Tang for their continuous interest and helpful conversation on this work.

2. Preliminaries. Let $N^{n}$ be a compact oriented homogeneous isoparametric hypersurface immersed in the standard unit sphere $S^{n+1}(1) \subset \mathbf{R}^{n+1}$ with $g$ distinct principal curvatures, where $g$ must be 1, 2, 3, 4 or 6 ([17]). Denote by $\mathbf{x}$ its position vector of a point of $N$ and $\mathbf{n}$ the unit normal vector field of $N$ in $S^{n+1}(1)$. Then the image $\mathcal{G}\left(N^{n}\right)$ of the Gauss map defined by

$$
\mathcal{G}: N^{n} \ni p \longmapsto \mathbf{x}(p) \wedge \mathbf{n}(p) \cong[\mathbf{x}(p)+\sqrt{-1} \mathbf{n}(p)] \in \widetilde{G r} r_{2}\left(\mathbf{R}^{n+2}\right) \cong Q_{n}(\mathbf{C})
$$

is a compact minimal Lagrangian submanifold embedded in the complex hyperquadric $Q_{n}(\mathbf{C})$ with the Deck transformation group $\mathbf{Z}_{g}$. Let $g_{Q_{n}(\mathbf{C})}^{\text {std }}$ be the standard Kähler metric of $Q_{n}(\mathbf{C})$ induced from the standard inner product of $\mathbf{R}^{n+2}$. Note that the Einstein constant of $g_{Q_{n}}^{\text {std }}$ (C) is equal to $n$. The Gauss image $\mathcal{G}\left(N^{n}\right) \subset Q_{n}(\mathbf{C})$ is Hamiltonian stable if and only if the first (positive) eigenvalue of $\mathcal{G}\left(N^{n}\right)$ is $n$ (cf. [2]). By Hsiang-Lawson ([9]) and Takagi-Takahashi ([22]), any homogeneous isoparametric hypersurface in a sphere can be obtained as a principal orbit of the isotropy representation of a compact Riemannian symmetric pair $(U, K)$ of rank 2 . Let $\mathfrak{u}=\mathfrak{k}+\mathfrak{p}$ be the canonical decomposition of $\mathfrak{u}$ as a symmetric Lie algebra of a symmetric pair $(U, K)$ of rank 2 and $\mathfrak{a}$ be a maximal abelian subspace of $\mathfrak{p}$. Define an $\operatorname{Ad} U$-invariant 
inner product $\langle,\rangle_{\mathfrak{u}}$ of $\mathfrak{u}$ from the Killing-Cartan form of $\mathfrak{u}$. Then the vector space $\mathfrak{p}$ equipped with the inner product $\langle,\rangle_{\mathfrak{u}}$ can be identified with the Euclidean space $\mathbf{R}^{n+2}$ and $S^{n+1}(1)$ denotes the $(n+1)$-dimensional unit standard sphere in $\mathfrak{p}$. The linear isotropy action $\operatorname{Ad}_{\mathfrak{p}}$ of $K$ on $\mathfrak{p}$ and thus on $S^{n+1}(1)$ induces the group action of $K$ on $\widetilde{G r}_{2}(\mathfrak{p}) \cong Q_{n}(\mathbf{C})$. For each regular element $H$ of $\mathfrak{a} \cap S^{n+1}(1)$, we get a homogeneous isoparametric hypersurface in the unit sphere

$$
N^{n}=\left(\operatorname{Ad}_{\mathfrak{p}} K\right) H \subset S^{n+1}(1) \subset \mathfrak{p} \cong \mathbf{R}^{n+2} .
$$

Its Gauss image is

$$
L^{n}=\mathcal{G}\left(N^{n}\right)=K \cdot[\mathfrak{a}]=\left[\left(\operatorname{Ad}_{\mathfrak{p}} K\right) \mathfrak{a}\right] \subset \widetilde{G} r_{2}(\mathfrak{p}) \cong Q_{n}(\mathbf{C}) .
$$

Here $N$ and $\mathcal{G}\left(N^{n}\right)$ have homogeneous space expressions $N \cong K / K_{0}$ and $\mathcal{G}\left(N^{n}\right) \cong K / K_{[\mathfrak{a}]}$, where we define

$$
\begin{aligned}
K_{0} & :=\left\{k \in K \mid \operatorname{Ad}_{\mathfrak{p}}(k)(H)=H\right\} \\
& =\left\{k \in K \mid \operatorname{Ad}_{\mathfrak{p}}(k)(H)=H \text { for each } H \in \mathfrak{a}\right\}, \\
K_{\mathfrak{a}} & :=\left\{k \in K \mid \operatorname{Ad}_{\mathfrak{p}}(k)(\mathfrak{a})=\mathfrak{a}\right\}, \\
K_{[\mathfrak{a}]} & :=\left\{k \in K_{\mathfrak{a}} \mid \operatorname{Ad}_{\mathfrak{p}}(k): \mathfrak{a} \rightarrow \mathfrak{a} \text { preserves the orientation of } \mathfrak{a}\right\} .
\end{aligned}
$$

Let $\Sigma(U, K)$ be the set of (restricted) roots of $(\mathfrak{u}, \mathfrak{k})$ and $\Sigma^{+}(U, K)$ be its subset of positive roots. We have the following root space decompositions of $\mathfrak{k}$ and $\mathfrak{p}$ as follows:

$$
\mathfrak{k}=\mathfrak{k}_{0}+\sum_{\gamma \in \Sigma^{+}(U, K)} \mathfrak{k}_{\gamma}, \quad \mathfrak{p}=\mathfrak{a}+\sum_{\gamma \in \Sigma^{+}(U, K)} \mathfrak{p}_{\gamma},
$$

where

$$
\begin{aligned}
\mathfrak{k}_{0} & :=\{X \in \mathfrak{k} \mid[X, \mathfrak{a}] \subset \mathfrak{a}\} \\
& =\{X \in \mathfrak{k} \mid[X, H]=0 \quad \text { for each } H \in \mathfrak{a}\}, \\
\mathfrak{k}_{\gamma} & :=\left\{X \in \mathfrak{k} \mid(\operatorname{ad} H)^{2} X=(\gamma(H))^{2} X \text { for each } H \in \mathfrak{a}\right\}, \\
\mathfrak{p}_{\gamma} & :=\left\{Y \in \mathfrak{p} \mid(\operatorname{ad} H)^{2} Y=(\gamma(H))^{2} Y \text { for each } H \in \mathfrak{a}\right\} .
\end{aligned}
$$

For each $\gamma \in \Sigma^{+}(U, K)$, set $m(\gamma):=\operatorname{dim} \mathfrak{k}_{\gamma}=\operatorname{dim} \mathfrak{p}_{\gamma}$. Define

$$
\mathfrak{m}:=\sum_{\gamma \in \Sigma^{+}(U, K)} \mathfrak{k}_{\gamma} \quad \text { and } \quad \mathfrak{a}^{\perp}:=\sum_{\gamma \in \Sigma^{+}(U, K)} \mathfrak{p}_{\gamma} .
$$

Then the tangent vector spaces $T_{e K_{0}}\left(K / K_{0}\right)$ and $T_{e K_{[\mathfrak{a}]}}\left(K / K_{[\mathfrak{a}]}\right)$ can be identified with the vector subspace $\mathfrak{m}$ of $\mathfrak{k}$. We can choose an orthonormal basis of $\mathfrak{m}$ and $\mathfrak{a}^{\perp}$ with respect to $\langle,\rangle_{\mathfrak{u}}$

$$
\left\{X_{\gamma, i} \in \mathfrak{k}_{\gamma} \mid \gamma \in \Sigma^{+}(U, K), i=1,2, \ldots, m(\gamma)\right\}
$$

and

$$
\left\{Y_{\gamma, i} \in \mathfrak{p}_{\gamma} \mid \gamma \in \Sigma^{+}(U, K), i=1,2, \ldots, m(\gamma)\right\}
$$

such that

$$
\left[H, X_{\gamma, i}\right]=\sqrt{-1} \gamma(H) Y_{\gamma, i}, \quad\left[H, Y_{\gamma, i}\right]=-\sqrt{-1} \gamma(H) X_{\gamma, i}
$$


for each $H \in \mathfrak{a}$. Let $\langle$,$\rangle denote the \operatorname{Ad}_{\mathfrak{m}}\left(K_{0}\right)$-invariant inner product of $\mathfrak{m}$ corresponding to the induced metric $\mathcal{G}^{*} g_{Q_{n}(\mathbf{C})}^{\text {std }}$ on $K / K_{0}$. Thus we know ([12]) that

$$
\left\{\frac{1}{\|\gamma\|_{\mathfrak{u}}} X_{\gamma, i} \mid \gamma \in \Sigma^{+}(U, K), i=1,2, \ldots, m(\gamma)\right\}
$$

is an orthonormal basis of $\mathfrak{m}$ relative to $\langle$,$\rangle .$

The Laplace operator $\triangle_{L^{n}}^{0}=\delta d$ acting on $C^{\infty}\left(K / K_{0}, \mathbf{C}\right)$ with respect to the induced metric $\mathcal{G}^{*} g_{Q_{n}(\mathbf{C})}^{\text {std }}$ corresponds to the linear differential operator $-\mathcal{C}_{L^{n}}$ on $C^{\infty}(K, \mathbf{C})_{K_{0}}$, where $\mathcal{C}_{L^{n}}$ is the Casimir operator relative to the $\operatorname{Ad}_{\mathfrak{m}}\left(K_{0}\right)$-invariant inner product $\langle$,$\rangle of \mathfrak{m}$ defined by

$$
\mathcal{C}_{L^{n}}:=\sum_{\gamma \in \Sigma^{+}(U, K)} \sum_{i=1}^{m(\gamma)} \frac{1}{\|\gamma\|_{\mathfrak{u}}^{2}}\left(X_{\gamma, i}\right)^{2} .
$$

Suppose that $\Sigma(U, K)$ is irreducible. Let $\gamma_{0}$ denote the highest root of $\Sigma(U, K)$. For $g=$ 3,4 , or 6 , the restricted root system $\Sigma(U, K)$ is of type $A_{2}, B_{2}, B C_{2}$ or $G_{2}$. Then for each $\gamma \in \Sigma^{+}(U, K)$,

$$
\frac{\|\gamma\|_{\mathfrak{u}}^{2}}{\left\|\gamma_{0}\right\|_{\mathfrak{u}}^{2}}= \begin{cases}1 & \text { if } \Sigma(U, K) \text { is of type } A_{2}, \\ 1 \text { or } 1 / 3 & \text { if } \quad \Sigma(U, K) \text { is of type } G_{2}, \\ 1 \text { or } 1 / 2 & \text { if } \quad \Sigma(U, K) \text { is of type } B_{2}, \\ 1,1 / 2 \text { or } 1 / 4 & \text { if } \quad \Sigma(U, K) \text { is of type } B C_{2} .\end{cases}
$$

Set

$$
\Sigma_{1}^{+}(U, K):=\left\{\gamma \in \Sigma^{+}(U, K) \mid\|\gamma\|_{\mathfrak{u}}^{2}=\left\|\gamma_{0}\right\|_{\mathfrak{u}}^{2}\right\} .
$$

Define a symmetric Lie subalgebra $\left(\mathfrak{u}_{1}, \mathfrak{k}_{1}\right)$ by

$$
\begin{aligned}
\mathfrak{k}_{1} & :=\mathfrak{k}_{0}+\sum_{\gamma \in \Sigma_{1}^{+}(U, K)} \mathfrak{k}_{\gamma}, \quad \mathfrak{p}_{1}:=\mathfrak{a}+\sum_{\gamma \in \Sigma_{1}^{+}(U, K)} \mathfrak{p}_{\gamma}, \\
\mathfrak{u}_{1} & :=\mathfrak{k}_{1}+\mathfrak{p}_{1} .
\end{aligned}
$$

Let $K_{1}$ and $U_{1}$ denote connected compact Lie subgroups of $K$ and $U$ generated by $\mathfrak{k}_{1}$ and $\mathfrak{u}_{1}$.

Suppose that $\Sigma^{+}(U, K)$ is of type $B C_{2}$. Define

$$
\Sigma_{2}^{+}(U, K):=\left\{\gamma \in \Sigma^{+}(U, K) \mid\|\gamma\|_{\mathfrak{u}}^{2}=\left\|\gamma_{0}\right\|_{\mathfrak{u}}^{2} \text { or }\left\|\gamma_{0}\right\|_{\mathfrak{u}}^{2} / 2\right\} .
$$

Define a symmetric Lie subalgebra $\left(\mathfrak{u}_{2}, \mathfrak{k}_{2}\right)$ by

$$
\begin{aligned}
\mathfrak{k}_{2} & :=\mathfrak{k}_{0}+\sum_{\gamma \in \Sigma_{2}^{+}(U, K)} \mathfrak{k}_{\gamma}, \quad \mathfrak{p}_{2}:=\mathfrak{a}+\sum_{\gamma \in \Sigma_{2}^{+}(U, K)} \mathfrak{p}_{\gamma}, \\
\mathfrak{u}_{2} & :=\mathfrak{k}_{2}+\mathfrak{p}_{2} .
\end{aligned}
$$


Let $K_{2}$ and $U_{2}$ denote connected compact Lie subgroups of $K$ and $U$ generated by $\mathfrak{k}_{2}$ and $\mathfrak{u}_{2}$. We have the following subgroups of $K$ in each case:

$$
\begin{array}{ll}
K_{0} \subset K, & \text { if } \Sigma(U, K) \text { is of type } A_{2} ; \\
K_{0} \subset K_{1} \subset K, & \text { if } \Sigma(U, K) \text { is of type } B_{2} \text { or } G_{2} ; \\
K_{0} \subset K_{1} \subset K_{2} \subset K, & \text { if } \Sigma(U, K) \text { is of type } B C_{2} .
\end{array}
$$

Set

$$
\begin{aligned}
\mathcal{C}_{K / K_{0},\langle,\rangle_{\mathfrak{u}}} & :=\sum_{\gamma \in \Sigma^{+}(U, K)} \sum_{i=1}^{m(\gamma)}\left(X_{\gamma, i}\right)^{2}, \\
\mathcal{C}_{\left.K_{1} / K_{0}, \zeta,\right\rangle_{\mathfrak{u}}}:= & \sum_{\gamma \in \Sigma_{1}^{+}(U, K)} \sum_{i=1}^{m(\gamma)}\left(X_{\gamma, i}\right)^{2}, \\
\mathcal{C}_{\left.K_{2} / K_{0}, \zeta,\right\rangle_{\mathfrak{u}}}:= & \sum_{\gamma \in \Sigma_{2}^{+}(U, K)} \sum_{i=1}^{m(\gamma)}\left(X_{\gamma, i}\right)^{2} .
\end{aligned}
$$

Then the Casimir operator $\mathcal{C}_{L^{n}}$ can be decomposed as follows:

LEMMA 2.1.

$$
\mathcal{C}_{L^{n}}=\left\{\begin{array}{l}
\frac{1}{\left\|\gamma_{0}\right\|_{\mathfrak{u}}^{2}} \mathcal{C}_{K / K_{0},\langle,\rangle_{\mathfrak{u}}} \text { if } \Sigma(U, K) \text { is of type } A_{2} ; \\
\frac{3}{\left\|\gamma_{0}\right\|_{\mathfrak{u}}^{2}} \mathcal{C}_{K / K_{0},\langle,\rangle_{\mathfrak{u}}}-\frac{2}{\left\|\gamma_{0}\right\|_{\mathfrak{u}}^{2}} \mathcal{C}_{K_{1} / K_{0},\langle,\rangle_{\mathfrak{u}}} \text { if } \Sigma(U, K) \text { is of type } G_{2} ; \\
\frac{2}{\left\|\gamma_{0}\right\|_{\mathfrak{u}}^{2}} \mathcal{C}_{K / K_{0},\langle,\rangle_{\mathfrak{u}}}-\frac{1}{\left\|\gamma_{0}\right\|_{\mathfrak{u}}^{2}} \mathcal{C}_{K_{1} / K_{0},\langle,\rangle_{\mathfrak{u}}} \text { if } \Sigma(U, K) \text { is of type } B_{2} ; \\
\frac{4}{\left\|\gamma_{0}\right\|_{\mathfrak{u}}^{2}} \mathcal{C}_{K / K_{0},\langle,\rangle_{\mathfrak{u}}}-\frac{2}{\left\|\gamma_{0}\right\|_{\mathfrak{u}}^{2}} \mathcal{C}_{K_{2} / K_{0},\langle,\rangle_{\mathfrak{u}}}-\frac{1}{\left\|\gamma_{0}\right\|_{\mathfrak{u}}^{2}} \mathcal{C}_{K_{1} / K_{0},\langle,\rangle_{\mathfrak{u}}} \\
\text { if } \Sigma(U, K) \text { is of type } B C_{2} .
\end{array}\right.
$$

Moreover, the Casimir operators $\mathcal{C}_{K / K_{0}}, \mathcal{C}_{K_{1} / K_{0}}\left(\right.$ and $\left.\mathcal{C}_{K_{2} / K_{0}}\right)$ commute with each other

For homogeneous isoparametric hypersurfaces of exceptional type with $g=6$ or 4 distinct principal curvatures, we have the following interesting fibrations on homogeneous isoparametric hypersurfaces by lower dimensional homogeneous isoparametric hypersurfaces, which give rise to the decompositions of the Casimir operators.

(i) In the case $g=6$ and $(U, K)=\left(G_{2}, S O(4)\right),\left(m_{1}, m_{2}\right)=(1,1)$. 


$$
\begin{gathered}
N^{6}=K / K_{0}=S O(4) /\left(\mathbf{Z}_{2}+\mathbf{Z}_{2}\right) \\
K_{1} / K_{0}=S O(3) /\left(\mathbf{Z}_{2}+\mathbf{Z}_{2}\right) \\
K / K_{1}=S O(4) / S O(3) \cong S^{3}
\end{gathered}
$$

(ii) In the case $g=6$ and $(U, K)=\left(G_{2} \times G_{2}, G_{2}\right),\left(m_{1}, m_{2}\right)=(2,2)$.

$$
\begin{aligned}
& N^{12}=K / K_{0}=G_{2} / T^{2} \\
& K_{1} / K_{0}=S U(3) / T^{2} \\
& K / K_{1}=G_{2} / S U(3) \cong S^{6}
\end{aligned}
$$

(iii) In the case $g=4$ and $(U, K)=\left(E_{6}, U(1) \cdot \operatorname{Spin}(10)\right),\left(m_{1}, m_{2}\right)=(6,9)$.

$$
\begin{aligned}
& N^{30}=\frac{K}{K_{0}}=\frac{U(1) \cdot \operatorname{Spin}(10)}{S^{1} \cdot \operatorname{Spin}(6)} \\
& \stackrel{K}{\longrightarrow} \quad \frac{K}{K_{0}}=\frac{U(1) \cdot \operatorname{Spin}(10)}{S^{1} \cdot \operatorname{Spin}(6)} \\
& \mid \begin{array}{c|c}
\frac{K_{1}}{K_{0}}=\frac{S^{1} \cdot(\operatorname{Spin}(2) \cdot(\operatorname{Spin}(2) \cdot \operatorname{Spin}(6)))}{S^{1} \cdot \operatorname{Spin}(6)} & \frac{K_{2}}{K_{0}}=\frac{U(1) \cdot(\operatorname{Spin}(2) \cdot \operatorname{Spin}(8))}{S^{1} \cdot \operatorname{Spin}(6)} \\
\frac{K_{2}}{K_{1}}=\frac{U(1) \cdot(\operatorname{Spin}(2) \cdot \operatorname{Spin}(8))}{S^{1} \cdot(\operatorname{Spin}(2) \cdot(\operatorname{Spin}(2) \cdot \operatorname{Spin}(6))} &
\end{array} \\
& \frac{K}{K_{1}}=\frac{U(1) \cdot \operatorname{Spin}(10)}{S^{1} \cdot(\operatorname{Spin}(2) \cdot(\operatorname{Spin}(2) \cdot \operatorname{Spin}(6)))} \quad \longrightarrow \quad \frac{K}{K_{2}}=\frac{U(1) \cdot \operatorname{Spin}(10)}{U(1) \cdot(\operatorname{Spin}(2) \cdot \operatorname{Spin}(8))}
\end{aligned}
$$

3. The case $(U, K)=\left(G_{2} \times G_{2}, G_{2}\right)$. Let $U=G_{2} \times G_{2}, K=\{(x, x) \in U \mid x \in$ $\left.G_{2}\right\}$ and $(U, K)$ is of type $G_{2}$. Then $K_{0}=\{k \in K \mid \operatorname{Ad}(k) H=H$ for each $H \in \mathfrak{a}\} \cong T^{2}$ is a maximal torus of $G_{2}$ and $N^{12}=K / K_{0} \cong G_{2} / T^{2}$ is a maximal flag manifold of dimension $n=12$. Thus its Gauss image is $L^{12}=\mathcal{G}\left(N^{12}\right)\left(\cong N^{12} / \mathbf{Z}_{6}\right)=K \cdot[\mathfrak{a}] \cong\left(K / K_{[\mathfrak{a}]}\right) \subset$ $Q_{12}(\mathbf{C})$.

Set $\langle,\rangle_{\mathfrak{u}}=-B_{\mathfrak{u}}\left(\right.$, ), where $B_{\mathfrak{u}}($,$) denotes the Killing-Cartan form of \mathfrak{u}$. Let $\langle$,$\rangle be the$ inner product of $\mathfrak{m}$ corresponding to the invariant induced metric on $L^{n}$ from $\left(Q_{n}(\mathbf{C}), g_{Q_{n}(\mathbf{C})}^{\text {std }}\right)$.

The restricted root system $\Sigma(U, K)$ of type $G_{2}$, can be given as follows ([5]):

$$
\begin{aligned}
\Sigma(U, K)= & \pm\left(\varepsilon_{1}-\varepsilon_{2}\right)= \pm \alpha_{1}, \pm\left(\varepsilon_{3}-\varepsilon_{1}\right)= \pm\left(\alpha_{1}+\alpha_{2}\right), \\
& \pm\left(\varepsilon_{3}-\varepsilon_{2}\right)= \pm\left(2 \alpha_{1}+\alpha_{2}\right), \pm\left(-2 \varepsilon_{1}+\varepsilon_{2}+\varepsilon_{3}\right)= \pm \alpha_{2}, \\
& \pm\left(\varepsilon_{1}-2 \varepsilon_{2}+\varepsilon_{3}\right)= \pm\left(3 \alpha_{1}+\alpha_{2}\right), \\
& \left. \pm\left(2 \varepsilon_{3}-\varepsilon_{1}-\varepsilon_{2}\right)= \pm\left(3 \alpha_{1}+2 \alpha_{2}\right)=\tilde{\alpha}\right\},
\end{aligned}
$$


where $\Pi(U, K)=\left\{\alpha_{1}=\varepsilon_{1}-\varepsilon_{2}, \alpha_{2}=-2 \varepsilon_{1}+\varepsilon_{2}+\varepsilon_{3}\right\}$ is its fundamental root system. Here

$$
\|\gamma\|_{\mathfrak{u}}^{2}= \begin{cases}\frac{1}{24} & \text { if } \gamma \text { is short } \\ \frac{1}{8} & \text { if } \gamma \text { is long. }\end{cases}
$$

Now $K_{1}=S U(3)$ and $K_{0}=T^{2} \subset K_{1}=S U(3) \subset K=G_{2}$.

In Lemma 2.1 the Casimir operator

$$
\mathcal{C}_{L}=\frac{3}{\left\|\gamma_{0}\right\|_{\mathfrak{u}}^{2}} \mathcal{C}_{K / K_{0},\langle,\rangle_{\mathfrak{u}}}-\frac{2}{\left\|\gamma_{0}\right\|_{\mathfrak{u}}^{2}} \mathcal{C}_{K_{1} / K_{0},\langle,\rangle_{\mathfrak{u}}},
$$

of $L^{n}$ with respect to $\langle$,$\rangle corresponding to -\Delta_{L^{12}}$ becomes

$$
\begin{aligned}
\mathcal{C}_{L} & =24 \mathcal{C}_{K / K_{0},\langle,\rangle_{\mathfrak{u}}}-16 \mathcal{C}_{K_{1} / K_{0},\langle,\rangle_{\mathfrak{u}}} \\
& =12 \mathcal{C}_{K / K_{0}}^{\mathfrak{k}}-8 \mathcal{C}_{K_{1} / K_{0}}^{\mathfrak{k}} \\
& =12 \mathcal{C}_{K / K_{0}}^{\mathfrak{k}}-6 \mathcal{C}_{K_{1} / K_{0}}^{\mathfrak{k}_{1}},
\end{aligned}
$$

where $\mathcal{C}_{K / K_{0}}^{\mathfrak{k}}$ and $\mathcal{C}_{K_{1} / K_{0}}^{\mathfrak{k}}$ denote the Casimir operators of $K / K_{0}$ and $K_{1} / K_{0}$ relative to the $K_{0}$ invariant inner product induced from the Killing-Cartan form of $\mathfrak{k}$, respectively, and $\mathcal{C}_{K_{1} / K_{0}}^{\mathfrak{k}_{1}}$ denotes the Casimir operator of $K_{1} / K_{0}$ relative to the $K_{0}$-invariant inner product induced from the Killing-Cartan form of $\mathfrak{k}_{1}$.

Let $\left\{\alpha_{1}, \alpha_{2}\right\}$ be the fundamental root system of $G_{2}$ and $\left\{\Lambda_{1}, \Lambda_{2}\right\}$ be the fundamental weight system of $G_{2}$. In our work we frequently use Satoru Yamaguchi's results ([26]) on the spectra of maximal flag manifolds.

LEMMA 3.1 (Branching law of $\left.\left(G_{2}, T^{2}\right)[26]\right)$.

$$
\begin{aligned}
D\left(K, K_{0}\right) & =D\left(G_{2}, T^{2}\right)=D\left(G_{2}\right) \\
& =\left\{\Lambda=m_{1} \Lambda_{1}+m_{2} \Lambda_{2} \mid m_{1}, m_{2} \in \mathbf{Z}, m_{1} \geq 0, m_{2} \geq 0\right\} \\
& =\left\{\Lambda=p_{1} \alpha_{1}+p_{1} \alpha_{2} \mid p_{1}, p_{2} \in \mathbf{Z}, p_{1} \geq 1, p_{2} \geq 1\right\} .
\end{aligned}
$$

The eigenvalue formula of the Casimir operator $\mathcal{C}_{K / K_{0}}$ relative to the inner product induced from the Killing-Cartan form of $G_{2}$ is

$$
-c\left(\Lambda,\langle,\rangle_{\mathfrak{g}_{2}}\right)=\frac{1}{24}\left(m_{1} p_{1}+3 m_{2} p_{2}+2 p_{1}+6 p_{2}\right)
$$

for each $\Lambda \in D\left(G_{2}, T^{2}\right)=D\left(G_{2}\right)$.

Since

$$
-\mathcal{C}_{L}=-\left(4 \mathcal{C}_{K / K_{0}}^{\mathfrak{g}_{2}}+\sum_{\gamma: \text { short }} 16\left(X_{\gamma, i}\right)^{2}\right) \geq-4 \mathcal{C}_{K / K_{0}}^{\mathfrak{g}_{2}},
$$

if the eigenvalue $-c_{L}$ of $-\mathcal{C}_{L}$ satisfies $-c_{L} \leq n=12$, then $-c_{\Lambda} \leq 3$. 
By using the formula (10), we obtain

$$
\begin{aligned}
\{\Lambda \in D( & \left.\left.G_{2}, T^{2}\right) \mid-c\left(\Lambda,\langle,\rangle_{\mathfrak{g}_{2}}\right) \leq 3\right\} \\
= & \left\{0, \Lambda_{1}\left(\left(p_{1}, p_{2}\right)=(2,1)\right), 2 \Lambda_{1}\left(\left(p_{1}, p_{2}\right)=(4,2)\right), 3 \Lambda_{1}\left(\left(p_{1}, p_{2}\right)=(6,3)\right),\right. \\
& \Lambda_{2}\left(\left(p_{1}, p_{2}\right)=(3,2)\right), 2 \Lambda_{2}\left(\left(p_{1}, p_{2}\right)=(6,4)\right), \Lambda_{1}+\Lambda_{2}\left(\left(p_{1}, p_{2}\right)=(5,3)\right), \\
& \left.2 \Lambda_{1}+\Lambda_{2}\left(\left(p_{1}, p_{2}\right)=(7,4)\right)\right\} .
\end{aligned}
$$

Let $\left\{\alpha_{1}^{\prime}, \alpha_{2}^{\prime}\right\}$ be the fundamental root system of $S U(3)$ and $\left\{\Lambda_{1}^{\prime}, \Lambda_{2}^{\prime}\right\}$ be the fundamental weight system of $S U(3)$. For each $\Lambda \in D\left(G_{2}, T^{2}\right)$ with $-c\left(\Lambda,\langle,\rangle_{\mathfrak{g}_{2}}\right) \leq 3$, by using the branching law of $\left(G_{2}, S U(3)\right)$ in [15], we can determine all irreducible $S U$ (3)-submodule $V_{\Lambda^{\prime}}$ with the highest weight $\Lambda^{\prime}=m_{1}^{\prime} \Lambda_{1}^{\prime}+m_{2} \Lambda_{2}^{\prime}$ contained in an irreducible $G_{2}$-module $V_{\Lambda}$ as in Table 1.

Since

$$
\begin{aligned}
& \mathfrak{g}_{2}^{\mathbf{C}}=\mathfrak{t}^{\mathbf{C}}+\sum_{\alpha \in \Sigma\left(G_{2}\right)} \mathfrak{g}^{\alpha}=\mathfrak{t}^{\mathbf{C}}+\sum_{\alpha: \text { short }} \mathfrak{g}^{\alpha}+\sum_{\alpha: \text { long }} \mathfrak{g}^{\alpha}, \\
& \mathfrak{s u ( 3 )} \mathbf{C}^{\mathbf{C}}=\mathfrak{t}^{\mathbf{C}}+\sum_{\alpha: \text { long }} \mathfrak{g}^{\alpha},
\end{aligned}
$$

we know that

$$
\begin{aligned}
T^{2} \cdot \mathbf{Z}_{6} & =\left\{a \in G_{2} \mid \operatorname{Ad}(a)(\mathfrak{t})=\mathfrak{t} \text { preserving the orientation of } \mathfrak{t}\right\} \\
& \supset\{a \in S U(3) \mid \operatorname{Ad}(a)(\mathfrak{t})=\mathfrak{t} \text { preserving the orientation of } \mathfrak{t}\} \\
& =T^{2} \cdot \mathbf{Z}_{3} .
\end{aligned}
$$

Now we use results on $S U(3) / T^{2}$, which were already treated in the case of $g=3$ and $m=2$ ([12]).

LEMMA 3.2 (Branching law of $\left.\left(S U(3), T^{2}\right)[26]\right)$.

$$
\begin{aligned}
D\left(K_{1}, K_{0}\right) & =D\left(S U(3), T^{2}\right) \\
& =\left\{\Lambda^{\prime}=m_{1}^{\prime} \Lambda_{1}^{\prime}+m_{2}^{\prime} \Lambda_{2}^{\prime} \mid m_{i}^{\prime} \in \mathbf{Z}, m_{i}^{\prime} \geq 0\right\} \\
& =\left\{\Lambda^{\prime}=p_{1}^{\prime} \alpha_{1}^{\prime}+p_{2}^{\prime} \alpha_{2}^{\prime} \mid p_{i}^{\prime} \in \mathbf{Z}, p_{i}^{\prime} \geq 1\right\},
\end{aligned}
$$

where

$$
m_{1}^{\prime}=2 p_{1}^{\prime}-p_{2}^{\prime} \geq 0, \quad m_{2}^{\prime}=-p_{1}^{\prime}+2 p_{2}^{\prime} \geq 0 .
$$

The eigenvalue formula is

$$
-c\left(\Lambda^{\prime},\langle,\rangle_{\mathfrak{s} u(3)}\right)=\frac{1}{6}\left(m_{1}^{\prime} p_{1}^{\prime}+m_{2}^{\prime} p_{2}^{\prime}+2 p_{1}^{\prime}+2 p_{2}^{\prime}\right)
$$

for each $\Lambda^{\prime} \in D\left(S U(3), T^{2}\right)$.

Using Lemma 3.2, we get that $\Lambda^{\prime}=m_{1}^{\prime} \Lambda_{1}^{\prime}+m_{2}^{\prime} \Lambda_{2}^{\prime} \in D\left(S U(3), T^{2}\right)$ such that $V_{\Lambda^{\prime}} \subset V_{\Lambda}$ for some $\Lambda \in D\left(G_{2}, T^{2}\right)$ with $-c\left(\Lambda,\langle,\rangle_{\mathfrak{g}_{2}}\right) \leq 3$ satisfies

$$
\left(m_{1}^{\prime}, m_{2}^{\prime}\right) \in\{(1,1),(3,0),(0,3),(2,2)\} .
$$

By using the formula (12), we compute the corresponding eigenvalues of $\mathcal{C}_{K_{1} / K_{0}}$ given in 
TABLE 1. All irreducible $S U(3)$-submodule $V_{\Lambda^{\prime}}$ contained in an irreducible $G_{2}$-module $V_{\Lambda}$.

\begin{tabular}{|c|c|c|c|l|}
\hline$\left(m_{1}, m_{2}\right)$ & $\left(p_{1}, p_{2}\right)$ & $-c$ & $\operatorname{dim}_{\mathbf{C}} V_{\Lambda}$ & irred. $S U(3)$-submodules $\left(m_{1}^{\prime}, m_{2}^{\prime}\right)$ \\
\hline$(1,0)$ & $(2,1)$ & $\frac{1}{2}$ & 7 & $(1,0),(0,1),(0,0)$ \\
\hline$(2,0)$ & $(4,2)$ & $\frac{7}{6}$ & 27 & $\begin{array}{l}(2,0),(1,1),(0,2),(1,0),(0,1), \\
(0,0)\end{array}$ \\
\hline$(3,0)$ & $(6,3)$ & 2 & 77 & $\begin{array}{l}(3,0),(2,1),(1,2),(0,3),(2,0), \\
(1,1),(0,2),(1,0),(0,1),(0,0)\end{array}$ \\
\hline$(0,1)$ & $(3,2)$ & 1 & 14 & $(1,1),(1,0),(0,1)$ \\
\hline$(0,2)$ & $(6,4)$ & $\frac{5}{2}$ & 77 & $\begin{array}{l}(2,2),(2,1),(1,2),(2,0),(1,1), \\
(0,2)\end{array}$ \\
\hline$(1,1)$ & $(5,3)$ & $\frac{7}{4}$ & 64 & $\begin{array}{l}(2,1),(1,2),(2,0), 2(1,1),(0,2), \\
(1,0),(0,1)\end{array}$ \\
\hline$(2,1)$ & $(7,4)$ & $\frac{8}{3}$ & 189 & $\begin{array}{l}(3,1),(2,2),(1,3),(3,0), 2(2,1),(0,3),(2,0), 2(1,1),(0,2), \\
(1,0),(0,1)\end{array}$ \\
\hline
\end{tabular}

TABLE 2. Eigenvalue computation for $\mathcal{C}_{K_{1} / K_{0}}$.

\begin{tabular}{|c|c|c|}
\hline$\left(p_{1}^{\prime}, p_{2}^{\prime}\right)$ & $\left(m_{1}^{\prime}, m_{2}^{\prime}\right)$ & $-c^{\prime}=-c\left(\Lambda^{\prime},\langle,\rangle_{\mathfrak{s} u(3)}\right)$ \\
\hline$(1,1)$ & $(1,1)$ & 1 \\
\hline$(2,1)$ & $(3,0)$ & 2 \\
\hline$(1,2)$ & $(0,3)$ & 2 \\
\hline$(2,2)$ & $(2,2)$ & $\frac{8}{3}$ \\
\hline
\end{tabular}

Table 2.

Therefore, for all $\Lambda \in D\left(G_{2}, T^{2}\right)$ and all $\Lambda^{\prime} \in D\left(S U(3), T^{2}\right)$ such that $V_{\Lambda^{\prime}} \subset V_{\Lambda}$ and $-c\left(\Lambda,\langle,\rangle_{\mathfrak{g}_{2}}\right) \leq 3$, the corresponding eigenvalues of $-\mathcal{C}_{L}=-12 \mathcal{C}_{K / K_{0}}^{\mathfrak{k}}+6 \mathcal{C}_{K_{1} / K_{0}}^{\mathfrak{k}_{1}}=$ $-12 c+6 c^{\prime}$ are given in Table 3:

Since $\Lambda_{1}^{\prime}+\Lambda_{2}^{\prime}\left(\left(m_{1}^{\prime}, m_{2}^{\prime}\right)=(1,1)\right)$ corresponds to the complexified adjoint representation of $S U(3)$, we see that $\left(V_{\Lambda_{1}^{\prime}+\Lambda_{2}^{\prime}}^{\prime}\right)_{T^{2}} \cong \mathfrak{t}^{2}$ and $\left(V_{\Lambda_{1}^{\prime}+\Lambda_{2}^{\prime}}^{\prime}\right)_{T^{2} \cdot \mathbf{Z}_{3}}=\{0\}$. Then

$$
\Lambda_{1}^{\prime}+\Lambda_{2}^{\prime} \notin D\left(S U(3), T^{2} \cdot \mathbf{Z}_{3}\right),
$$

and thus

$$
2 \Lambda_{1}, \Lambda_{2} \notin D\left(G_{2}, T^{2} \cdot \mathbf{Z}_{6}\right) .
$$

Now we obtain that $\mathcal{G}\left(G_{2} / T^{2}\right) \subset Q_{12}(\mathbf{C})$ is Hamiltonian stable. 
TABLE 3. Eigenvalues of $-\mathcal{C}_{L}$ for $L=G_{2} /\left(T_{2} \cdot \mathbf{Z}_{6}\right)$.

\begin{tabular}{|c|c|c|c|c|c|c|}
\hline$\left(m_{1}, m_{2}\right)$ & $\left(p_{1}, p_{2}\right)$ & $\operatorname{dim}_{\mathbf{C}} V_{\Lambda}$ & $-c$ & $\left(m_{1}^{\prime}, m_{2}^{\prime}\right)$ & $-c^{\prime}$ & $-12 c+6 c^{\prime}$ \\
\hline$(2,0)$ & $(4,2)$ & 27 & $\frac{7}{6}$ & $(1,1)$ & 1 & 8 \\
\hline$(3,0)$ & $(6,3)$ & 77 & 2 & $(1,1)$ & 1 & 18 \\
\hline$(3,0)$ & $(6,3)$ & 77 & 2 & $(3,0)$ & 2 & 12 \\
\hline$(3,0)$ & $(6,3)$ & 77 & 2 & $(0,3)$ & 2 & 12 \\
\hline$(0,1)$ & $(3,2)$ & 14 & 1 & $(1,1)$ & 1 & 6 \\
\hline$(0,2)$ & $(6,4)$ & 77 & $\frac{5}{2}$ & $(1,1)$ & 1 & 24 \\
\hline$(0,2)$ & $(6,4)$ & 77 & $\frac{5}{2}$ & $(2,2)$ & $\frac{8}{3}$ & 14 \\
\hline$(1,1)$ & $(5,3)$ & 64 & $\frac{7}{4}$ & $2(1,1)$ & 1 & 15 \\
\hline$(2,1)$ & $(7,4)$ & 189 & $\frac{8}{3}$ & $2(1,1)$ & 1 & 26 \\
\hline$(2,1)$ & $(7,4)$ & 189 & $\frac{8}{3}$ & $(3,0)$ & 2 & 20 \\
\hline$(2,1)$ & $(7,4)$ & 189 & $\frac{8}{3}$ & $(0,3)$ & 2 & 20 \\
\hline$(2,1)$ & $(7,4)$ & 189 & $\frac{8}{3}$ & $(2,2)$ & $\frac{8}{3}$ & 16 \\
\hline
\end{tabular}

We need to examine whether $3 \Lambda_{1} \in D\left(K, K_{[\mathfrak{a}]}\right)=D\left(G_{2}, T^{2} \cdot \mathbf{Z}_{6}\right)$ or not. Consider

$$
\left(V_{3 \Lambda_{1}}\right)_{T^{2}}=\left(V_{3 \Lambda_{1}^{\prime}}^{\prime}\right)_{T^{2}} \oplus\left(V_{3 \Lambda_{2}^{\prime}}^{\prime}\right)_{T^{2}} \oplus\left(V_{\Lambda_{1}^{\prime}+\Lambda_{2}^{\prime}}^{\prime}\right)_{T^{2}} .
$$

Since

$$
V_{3 \Lambda_{1}^{\prime}}^{\prime} \cong \operatorname{Sym}^{3}\left(\mathbf{C}^{3}\right)=\operatorname{span}_{\mathbf{C}}\left\{e_{i_{1}} \cdot e_{i_{2}} \cdot e_{i_{3}} \mid 1 \leq i_{1} \leq i_{2} \leq i_{3} \leq 3\right\},
$$

where $\left\{e_{1}, e_{2}, e_{3}\right\}$ is the standard basis of $\mathbf{C}^{3}$, we get

$$
\left(V_{3 \Lambda_{1}^{\prime}}^{\prime}\right)_{T^{2}}=\left(V_{3 \Lambda_{1}^{\prime}}^{\prime}\right)_{T^{2} \cdot \mathbf{Z}_{3}}=\operatorname{span}_{\mathbf{C}}\left\{e_{1} \cdot e_{2} \cdot e_{3}\right\} .
$$

Similarly, we get $V_{3 \Lambda_{2}^{\prime}}^{\prime} \cong \operatorname{Sym}^{3}\left(\overline{\mathbf{C}}^{3}\right)$ and $\left(V_{3 \Lambda_{2}^{\prime}}^{\prime}\right)_{T^{2}}=\left(V_{3 \Lambda_{2}^{\prime}}^{\prime}\right)_{T^{2} \cdot \mathbf{Z}_{3}}$ with dimension 1. On the other hand we know that $\left(V_{\Lambda_{1}^{\prime}+\Lambda_{2}^{\prime}}^{\prime}\right)_{T^{2}} \cong \mathfrak{t}$ and $\left(V_{\Lambda_{1}^{\prime}+\Lambda_{2}^{\prime}}^{\prime}\right)_{T^{2} \cdot \mathbf{Z}_{3}}=\{0\}$. Hence we get $\operatorname{dim}_{\mathbf{C}}\left(V_{3 \Lambda_{1}}\right)_{T^{2}}=4$ and $\operatorname{dim}_{\mathbf{C}}\left(V_{3 \Lambda_{1}}\right)_{T^{2} \cdot \mathbf{Z}_{3}}=2$. However $\operatorname{dim}_{\mathbf{C}}\left(V_{3 \Lambda_{1}}\right)_{T^{2} \cdot \mathbf{Z}_{6}}=1$. In fact, $T^{2} \cdot \mathbf{Z}_{6} \subset G_{2}, T^{2} \cdot \mathbf{Z}_{6} \not \subset S U(3), T^{2} \cdot \mathbf{Z}_{3} \subset S U(3)$ and $\left(T^{2} \cdot \mathbf{Z}_{6}\right) /\left(T^{2} \cdot \mathbf{Z}_{3}\right) \cong \mathbf{Z}_{2}$. Thus there exists an element $u \in T^{2} \cdot \mathbf{Z}_{6} \subset G_{2}$ with $u \notin S U(3)$ which satisfies $\operatorname{Ad}(u)(S U(3)) \subset$ $S U(3)$ and provides the generators of both $\left(T^{2} \cdot \mathbf{Z}_{6}\right) / T^{2} \cong \mathbf{Z}_{6}$ and $\left(T^{2} \cdot \mathbf{Z}_{6}\right) /\left(T^{2} \cdot \mathbf{Z}_{3}\right) \cong$ $\mathbf{Z}_{2}$. Then we observe that $\left.\rho_{3 \Lambda_{1}^{\prime}} \circ \operatorname{Ad}(u)\right|_{S U(3)} \cong \rho_{3 \Lambda_{2}^{\prime}}$ and $\rho_{3 \Lambda_{1}}(u)\left(V_{3 \Lambda_{1}^{\prime}}^{\prime}\right)=V_{3 \Lambda_{2}^{\prime}}^{\prime}$. Thus $\rho_{3 \Lambda_{1}}(u)\left(V_{3 \Lambda_{1}^{\prime}}^{\prime}\right)_{T^{2} \cdot \mathbf{Z}_{3}}=\left(V_{3 \Lambda_{2}^{\prime}}^{\prime}\right)_{T^{2} \cdot \mathbf{z}_{3}}$ and $\left.\left(\rho_{3 \Lambda_{1}}(u)\right)^{2}\right|_{\left(V_{3 \Lambda_{1}^{\prime}}^{\prime}\right)_{T^{2} \cdot \mathbf{z}_{3}}}=\left.\left(\rho_{3 \Lambda_{1}}\left(u^{2}\right)\right)\right|_{\left(V_{3 \Lambda_{1}^{\prime}}^{\prime}\right)_{T^{2} \cdot \mathbf{z}_{3}}}=$ Id, because $u^{2} \in T^{2} \cdot \mathbf{Z}_{3}$. Hence we have $\left(V_{3 \Lambda_{1}}\right)_{T^{2} \cdot \mathbf{Z}_{6}} \subset\left(V_{3 \Lambda_{1}^{\prime}}^{\prime}\right)_{T^{2} \cdot \mathbf{Z}_{3}} \oplus\left(V_{3 \Lambda_{2}^{\prime}}^{\prime}\right)_{T^{2} \cdot \mathbf{Z}_{3}}$ and $\operatorname{dim}\left(V_{3 \Lambda_{1}}\right)_{T^{2} \cdot \mathbf{Z}_{6}}=1$. Therefore $3 \Lambda_{1} \in D\left(G_{2}, T^{2} \cdot \mathbf{Z}_{6}\right)$ and its multiplicity is equal to 1 . It follows that

$$
n\left(L^{12}\right)=\operatorname{dim} \mathbf{C}\left(V_{3 \Lambda_{1}}\right)=77=91-14=\operatorname{dim}(S O(14))-\operatorname{dim}\left(G_{2}\right)=n_{h k}\left(L^{12}\right),
$$


that is, $\mathcal{G}\left(G_{2} / T^{2}\right) \subset Q_{12}(\mathbf{C})$ is Hamiltonian rigid.

Let $\wedge^{2} \mathbf{R}^{14}=\mathfrak{o}(n+2)=\operatorname{ad}_{\mathfrak{p}}\left(\mathfrak{g}_{2}\right)+\mathcal{V} \cong \mathfrak{g}_{2}+\mathcal{V}$. Then

$$
\bigwedge^{2} \mathbf{C}^{14}=\left(\bigwedge^{2} \mathbf{R}^{14}\right)^{\mathbf{C}}=\mathfrak{o}(n+2)^{\mathbf{C}}=\mathfrak{o}(n+2, \mathbf{C})=\operatorname{ad}_{\mathfrak{p}}\left(\mathfrak{g}_{2}^{\mathbf{C}}\right)+\mathcal{V}^{\mathbf{C}} \cong \mathfrak{g}_{2}^{\mathbf{C}}+\mathcal{V}^{\mathbf{C}}
$$

where $\operatorname{dim} \mathcal{V}=77$ and $\operatorname{dim}_{\mathbf{C}} \mathcal{V}^{\mathbf{C}}=77$. More precisely, we observe that $\mathcal{V}$ is a real 77dimensional irreducible $G_{2}$-module with $(\mathcal{V})_{T^{2} \cdot \mathbf{Z}_{6}} \neq\{0\}$, and $\mathcal{V}^{\mathbf{C}}$ is a complex 77-dimensional $G_{2}$-module with $(\mathcal{V})_{T^{2} \cdot \mathbf{z}_{6}}^{\mathbf{C}} \neq\{0\}$. Moreover, we have $\mathcal{V}^{\mathbf{C}} \cong V_{3 \Lambda_{1}}$ with $\operatorname{dim}_{\mathbf{C}}(\mathcal{V})_{T^{2} \cdot \mathbf{z}_{6}}^{\mathbf{C}}=1$.

From these arguments we conclude that

THEOREM 3.3. The Gauss image $L^{12}=\mathcal{G}\left(G_{2} / T^{2}\right)=G_{2} /\left(T^{2} \cdot \mathbf{Z}_{6}\right) \subset Q_{12}(\mathbf{C})$ is strictly Hamiltonian stable.

4. The case $(U, K)=\left(G_{2}, S O(4)\right)$. Let $U=G_{2}, K=S O(4)$ and $(U, K)$ is of type $G_{2}$. Let $\mathfrak{u}=\mathfrak{k}+\mathfrak{p}$ be the orthogonal symmetric Lie algebra of $\left(G_{2}, S O(4)\right)$ and $\mathfrak{a}$ be the maximal abelian subspace of $\mathfrak{p}$. Here $\mathfrak{u}=\mathfrak{g}_{2}, \mathfrak{k}=\mathfrak{s o}(4) \cong \mathfrak{s u}(2) \oplus \mathfrak{s u}(2)$. Let

$$
p: \widetilde{K}=\operatorname{Spin}(4)=S U(2) \times S U(2) \longrightarrow K=S O(4)
$$

be the universal covering Lie group homomorphism with kernel $\mathbf{Z}_{2}$.

Recall that the complete set of all inequivalent irreducible unitary representations of $S U(2)$ is given by

$$
\mathcal{D}(S U(2))=\left\{\left(V_{m}, \rho_{m}\right) \mid m \in \mathbf{Z}, m \geq 0\right\},
$$

where $V_{m}$ denotes the complex vector space of complex homogeneous polynomials of degree $m$ with two variables $z_{0}, z_{1}$ and the representation $\rho_{m}$ of $S U(2)$ on $V_{m}$ is defined by $\left(\rho_{m}(g) f\right)\left(z_{0}, z_{1}\right)=f\left(\left(z_{0}, z_{1}\right) g\right)$ for each $g \in S U(2)$. Set

$$
v_{k}^{(m)}\left(z_{0}, z_{1}\right):=\frac{1}{\sqrt{k !(m-k) !}} z_{0}^{m-k} z_{1}^{k} \in V_{m} \quad(k=0,1, \ldots, m)
$$

and define the standard Hermitian inner product of $V_{m}$ invariant under $\rho_{m}(S U(2))$ such that $\left\{v_{0}^{(m)}, \ldots, v_{m}^{(m)}\right\}$ is a unitary basis of $V_{m}$. Let $\left(V_{l} \otimes V_{m}, \rho_{l} \otimes \rho_{m}\right)$ denote an irreducible unitary representation of $S U(2) \times S U(2)$ of complex dimension $(l+1)(m+1)$ obtained by taking the exterior tensor product of $V_{l}$ and $V_{m}$ and then

$$
\left\{\left(V_{l} \otimes V_{m}, \rho_{l} \otimes \rho_{m}\right) \mid l, m \in \mathbf{Z}, l, m \geq 0\right\}
$$

is the complete set of all inequivalent irreducible unitary representations of $S U(2) \times S U(2)$.

The isotropy representation of $\left(G_{2}, S O(4)\right)$ is explicitly described as follows (cf. [8]): Suppose that $(l, m)=(3,1)$. The real 8-dimensional vector subspace $W$ of $V_{3} \otimes V_{1}$ spanned 
over $\mathbf{R}$ by

$$
\begin{gathered}
\left\{v_{0}^{(3)} \otimes v_{0}^{(1)}+v_{3}^{(3)} \otimes v_{1}^{(1)}, \sqrt{-1}\left(v_{0}^{(3)} \otimes v_{0}^{(1)}-v_{3}^{(3)} \otimes v_{1}^{(1)}\right),\right. \\
v_{1}^{(3)} \otimes v_{0}^{(1)}-v_{2}^{(3)} \otimes v_{1}^{(1)}, \sqrt{-1}\left(v_{1}^{(3)} \otimes v_{0}^{(1)}+v_{2}^{(3)} \otimes v_{1}^{(1)}\right), \\
v_{0}^{(3)} \otimes v_{1}^{(1)}-v_{3}^{(3)} \otimes v_{0}^{(1)}, \sqrt{-1}\left(v_{0}^{(3)} \otimes v_{1}^{(1)}+v_{3}^{(3)} \otimes v_{0}^{(1)}\right), \\
\left.v_{2}^{(3)} \otimes v_{0}^{(1)}+v_{1}^{(3)} \otimes v_{1}^{(1)}, \sqrt{-1}\left(v_{2}^{(3)} \otimes v_{0}^{(1)}-v_{1}^{(3)} \otimes v_{1}^{(1)}\right)\right\}
\end{gathered}
$$

gives an irreducible orthogonal representation of $S U(2) \times S U(2)$ whose complexification is $V_{3} \otimes V_{1}$, i.e., $\quad W$ is a real form of $V_{3} \otimes V_{1}$. Then the isotropy representation $\operatorname{Ad}_{\mathfrak{p}}$ of $\left(G_{2}, S O(4)\right)$ is given by $\operatorname{Ad}_{\mathfrak{p}}^{\mathbf{C}} \circ p \cong \rho_{3} \otimes \rho_{1}$ and the vector space $\mathfrak{p}$ is linearly isomorphic to $W$. Moreover $\mathfrak{a}$ corresponds to a vector subspace

$$
\mathbf{R}\left(v_{0}^{(3)} \otimes v_{0}^{(1)}+v_{3}^{(3)} \otimes v_{1}^{(1)}\right)+\mathbf{R}\left(v_{2}^{(3)} \otimes v_{0}^{(1)}+v_{1}^{(3)} \otimes v_{1}^{(1)}\right) .
$$
mula holds:

For each $X=\left(\begin{array}{cc}\sqrt{-1} x & u \\ -\bar{u} & -\sqrt{-1} x\end{array}\right), Y=\left(\begin{array}{cc}\sqrt{-1} y & w \\ -\bar{w} & -\sqrt{-1} y\end{array}\right) \in \mathfrak{s u}(2)$, the following useful for-

LEMMA 4.1.

$$
\begin{aligned}
{\left[d\left(\rho_{l} \otimes \rho_{m}\right)(\right.} & X, Y)]\left(v_{i}^{(l)} \otimes v_{j}^{(m)} \pm v_{l-i}^{(l)} \otimes v_{m-j}^{(m)}\right) \\
= & \{(2 i-l) x+(2 j-m) y\} \sqrt{-1}\left(v_{i}^{(l)} \otimes v_{j}^{(m)} \mp v_{l-i}^{(l)} \otimes v_{m-j}^{(m)}\right) \\
& -\sqrt{i(l-i+1)} \operatorname{Re}(u)\left(v_{i-1}^{(l)} \otimes v_{j}^{(m)} \mp v_{l-i+1}^{(l)} \otimes v_{m-j}^{(m)}\right) \\
& +\sqrt{i(l-i+1)} \operatorname{Im}(u) \sqrt{-1}\left(v_{i-1}^{(l)} \otimes v_{j}^{(m)} \pm v_{l-i+1}^{(l)} \otimes v_{m-j}^{(m)}\right) \\
& -\sqrt{j(m-j+1)} \operatorname{Re}(w)\left(v_{i}^{(l)} \otimes v_{j-1}^{(m)} \mp v_{l-i}^{(l)} \otimes v_{m-j+1}^{(m)}\right) \\
& +\sqrt{j(m-j+1)} \operatorname{Im}(w) \sqrt{-1}\left(v_{i}^{(l)} \otimes v_{j-1}^{(m)} \pm v_{l-i}^{(l)} \otimes v_{m-j+1}^{(m)}\right) \\
& +\sqrt{(l-i)(i+1)} \operatorname{Re}(u)\left(v_{i+1}^{(l)} \otimes v_{j}^{(m)} \mp v_{l-i-1}^{(l)} \otimes v_{m-j}^{(m)}\right) \\
& +\sqrt{(l-i)(i+1)} \operatorname{Im}(u) \sqrt{-1}\left(v_{i+1}^{(l)} \otimes v_{j}^{(m)} \pm v_{l-i-1}^{(l)} \otimes v_{m-j}^{(m)}\right) \\
& +\sqrt{(m-j)(j+1)} \operatorname{Re}(w)\left(v_{i}^{(l)} \otimes v_{j+1}^{(m)} \mp v_{l-i}^{(l)} \otimes v_{m-j-1}^{(m)}\right) \\
& +\sqrt{(m-j)(j+1)} \operatorname{Im}(w) \sqrt{-1}\left(v_{i}^{(l)} \otimes v_{j+1}^{(m)} \pm v_{l-i}^{(l)} \otimes v_{m-j-1}^{(m)}\right) .
\end{aligned}
$$

REMARK 4.2. By using the formula (14) we can check that the real vector subspace $W$ is invariant under the action of $S U(2) \times S U(2)$ via $\rho_{3} \otimes \rho_{1}$.

Define an orthonormal basis of the real vector space $W \cong \mathfrak{p}$ by

$$
\begin{aligned}
& H_{1}:=\frac{1}{\sqrt{2}}\left(v_{0}^{(3)} \otimes v_{0}^{(1)}+v_{3}^{(3)} \otimes v_{1}^{(1)}\right) \\
& H_{2}:=\frac{1}{\sqrt{2}}\left(v_{2}^{(3)} \otimes v_{0}^{(1)}+v_{1}^{(3)} \otimes v_{1}^{(1)}\right) \\
& E_{1}:=\frac{1}{\sqrt{2}} \sqrt{-1}\left(v_{0}^{(3)} \otimes v_{0}^{(1)}-v_{3}^{(3)} \otimes v_{1}^{(1)}\right)
\end{aligned}
$$




$$
\begin{aligned}
& E_{2}:=\frac{1}{\sqrt{2}}\left(v_{1}^{(3)} \otimes v_{0}^{(1)}-v_{2}^{(3)} \otimes v_{1}^{(1)}\right), \\
& E_{3}:=\frac{1}{\sqrt{2}} \sqrt{-1}\left(v_{1}^{(3)} \otimes v_{0}^{(1)}+v_{2}^{(3)} \otimes v_{1}^{(1)}\right), \\
& E_{4}:=\frac{1}{\sqrt{2}}\left(v_{0}^{(3)} \otimes v_{1}^{(1)}-v_{3}^{(3)} \otimes v_{0}^{(1)}\right), \\
& E_{5}:=\frac{1}{\sqrt{2}} \sqrt{-1}\left(v_{0}^{(3)} \otimes v_{1}^{(1)}+v_{3}^{(3)} \otimes v_{0}^{(1)}\right), \\
& E_{6}:=\frac{1}{\sqrt{2}} \sqrt{-1}\left(v_{2}^{(3)} \otimes v_{0}^{(1)}-v_{1}^{(3)} \otimes v_{1}^{(1)}\right) .
\end{aligned}
$$

Then we have the matrix expression as follows:

$\left[d\left(\rho_{3} \otimes \rho_{1}\right)(X, Y)\right]\left(H_{1}, H_{2}\right)$

$$
=\left(E_{1}, E_{2}, E_{3}, E_{4}, E_{5}, E_{6}\right)\left(\begin{array}{cc}
-(3 x+y) & 0 \\
\sqrt{3} \operatorname{Re}(u) & -(2 \operatorname{Re}(u)+\operatorname{Re}(w)) \\
\sqrt{3} \operatorname{Im}(u) & 2 \operatorname{Im}(u)+\operatorname{Im}(w) \\
\operatorname{Re}(w) & -\sqrt{3} \operatorname{Re}(u) \\
\operatorname{Im}(w) & \sqrt{3} \operatorname{Im}(u) \\
0 & x-y
\end{array}\right) .
$$

The inner product $\langle$,$\rangle corresponding to the metric induced from g_{Q_{6}(\mathbf{C})}^{\mathrm{std}}$ is given as follows: For $\left(X, X^{\prime}\right),\left(Y, Y^{\prime}\right) \in \mathfrak{s u}(2) \oplus \mathfrak{s u}(2)$,

$$
\begin{aligned}
\left\langle\left(X, X^{\prime}\right),\right. & \left.\left(Y, Y^{\prime}\right)\right\rangle \\
:= & \left(3 x+x^{\prime}\right)\left(3 y+y^{\prime}\right) \\
& +3 \operatorname{Re}(u) \operatorname{Re}(w)+\left(2 \operatorname{Re}(u)+\operatorname{Re}\left(u^{\prime}\right)\right)\left(2 \operatorname{Re}(w)+\operatorname{Re}\left(w^{\prime}\right)\right) \\
& +3 \operatorname{Im}(u) \operatorname{Im}(w)+\left(2 \operatorname{Im}(u)+\operatorname{Im}\left(u^{\prime}\right)\right)\left(2 \operatorname{Im}(w)+\operatorname{Im}\left(w^{\prime}\right)\right) \\
& +\operatorname{Re}\left(u^{\prime}\right) \operatorname{Re}\left(w^{\prime}\right)+3 \operatorname{Re}(u) \operatorname{Re}(w) \\
& +\operatorname{Im}\left(u^{\prime}\right) \operatorname{Im}\left(w^{\prime}\right)+3 \operatorname{Im}(u) \operatorname{Im}(w) \\
& +\left(x-x^{\prime}\right)\left(y-y^{\prime}\right) \\
= & 10 x y+2 x^{\prime} y+2 x y^{\prime}+2 x^{\prime} y^{\prime} \\
& +10 \operatorname{Re}(u) \operatorname{Re}(w)+2 \operatorname{Re}\left(u^{\prime}\right) \operatorname{Re}(w)+2 \operatorname{Re}(u) \operatorname{Re}\left(w^{\prime}\right)+2 \operatorname{Re}\left(u^{\prime}\right) \operatorname{Re}\left(w^{\prime}\right) \\
& +10 \operatorname{Im}(u) \operatorname{Im}(w)+2 \operatorname{Im}\left(u^{\prime}\right) \operatorname{Im}(w)+2 \operatorname{Im}(u) \operatorname{Im}\left(w^{\prime}\right)+2 \operatorname{Im}\left(u^{\prime}\right) \operatorname{Im}\left(w^{\prime}\right) .
\end{aligned}
$$

Thus the Casimir operator of $\left(\widetilde{K}, \widetilde{K}_{[\mathfrak{a}]}\right)$ relative to the inner product $\langle$,$\rangle is given as follows:$

$$
\begin{aligned}
\mathcal{C}_{L}= & \frac{1}{2}\left(X_{1}, 0\right) \cdot\left(X_{1}, 0\right)+\frac{1}{2}\left(X_{2}, 0\right) \cdot\left(X_{2}, 0\right)+\frac{1}{2}\left(X_{3}, 0\right) \cdot\left(X_{3}, 0\right) \\
& +\frac{5}{2}\left(0, X_{1}\right) \cdot\left(0, X_{1}\right)+\frac{5}{2}\left(0, X_{2}\right) \cdot\left(0, X_{2}\right)+\frac{5}{2}\left(0, X_{3}\right) \cdot\left(0, X_{3}\right) \\
& -\left(X_{1}, 0\right) \cdot\left(0, X_{1}\right)-\left(X_{2}, 0\right) \cdot\left(0, X_{2}\right)-\left(X_{3}, 0\right) \cdot\left(0, X_{3}\right),
\end{aligned}
$$

where

$$
X_{1}:=\frac{1}{2}\left(\begin{array}{cc}
\sqrt{-1} & 0 \\
0 & -\sqrt{-1}
\end{array}\right), \quad X_{2}:=\frac{1}{2}\left(\begin{array}{cc}
0 & 1 \\
-1 & 0
\end{array}\right), \quad X_{3}:=\frac{1}{2}\left(\begin{array}{cc}
0 & \sqrt{-1} \\
\sqrt{-1} & 0
\end{array}\right)
$$


is a basis of $\mathfrak{s u}(2)$ and $\left\{\left(X_{1}, 0\right),\left(X_{2}, 0\right),\left(X_{3}, 0\right),\left(0, X_{1}\right),\left(0, X_{2}\right),\left(0, X_{3}\right)\right\}$ is a basis of $\mathfrak{s u}(2) \oplus$ $\mathfrak{s u}(2)$. Hence, we have the following formula for the Casimir operator:

LEMMA 4.3.

$$
\begin{aligned}
& {\left[d\left(\rho_{l} \otimes \rho_{m}\right)\left(\mathcal{C}_{L}\right)\right]\left(v_{i}^{(l)} \otimes v_{a}^{(m)}\right) } \\
&=-\left\{\frac{l(l+2)}{8}+\frac{5 m(m+2)}{8}-\frac{(2 i-l)(4 a-m)}{4}\right\}\left(v_{i}^{(l)} \otimes v_{a}^{(m)}\right) \\
&+\frac{1}{2} \sqrt{(i+1)(l-i) a(m-a+1)}\left(v_{i+1}^{(l)} \otimes v_{a-1}^{(m)}\right) \\
&+\frac{1}{2} \sqrt{i(l-i+1)(a+1)(m-a)}\left(v_{i-1}^{(l)} \otimes v_{a+1}^{(m)}\right) .
\end{aligned}
$$

Set

$$
\widetilde{K}_{0}:=\{(A, B) \in \widetilde{K} \mid \operatorname{Ad}(p(A, B)) H=H \text { for each } H \in \mathfrak{a}\} .
$$

Then using this description of the isotropy representation we can compute directly

$$
\begin{aligned}
\widetilde{K}_{0}= & \left(\left(\begin{array}{ll}
1 & 0 \\
0 & 1
\end{array}\right),\left(\begin{array}{ll}
1 & 0 \\
0 & 1
\end{array}\right)\right),\left(\left(\begin{array}{cc}
\sqrt{-1} & 0 \\
0 & -\sqrt{-1}
\end{array}\right),\left(\begin{array}{cc}
\sqrt{-1} & 0 \\
0 & -\sqrt{-1}
\end{array}\right)\right), \\
& \left(\left(\begin{array}{cc}
-1 & 0 \\
0 & -1
\end{array}\right),\left(\begin{array}{cc}
-1 & 0 \\
0 & -1
\end{array}\right)\right),\left(\left(\begin{array}{cc}
-\sqrt{-1} & 0 \\
0 & \sqrt{-1}
\end{array}\right),\left(\begin{array}{cc}
-\sqrt{-1} & 0 \\
0 & \sqrt{-1}
\end{array}\right)\right), \\
& \left(\left(\begin{array}{cc}
0 & -1 \\
1 & 0
\end{array}\right),\left(\begin{array}{cc}
0 & -1 \\
1 & 0
\end{array}\right)\right),\left(\left(\begin{array}{cc}
0 & \sqrt{-1} \\
\sqrt{-1} & 0
\end{array}\right),\left(\begin{array}{cc}
0 & \sqrt{-1} \\
\sqrt{-1} & 0
\end{array}\right)\right) \\
& \left.\left(\left(\begin{array}{cc}
0 & 1 \\
-1 & 0
\end{array}\right),\left(\begin{array}{cc}
0 & 1 \\
-1 & 0
\end{array}\right)\right),\left(\left(\begin{array}{cc}
0 & -\sqrt{-1} \\
-\sqrt{-1} & 0
\end{array}\right),\left(\begin{array}{cc}
0 & -\sqrt{-1} \\
-\sqrt{-1} & 0
\end{array}\right)\right)\right\}
\end{aligned}
$$

In particular, the order of $\widetilde{K}_{0}$ is 8 . This result is consistent with thoes of [3, p.611], [4, p.651] and $[25$, p.573] in the topology of transformation group theory. Moreover we obtain

$$
\begin{aligned}
K_{0}= & \left\{p\left(\left(\begin{array}{ll}
1 & 0 \\
0 & 1
\end{array}\right),\left(\begin{array}{ll}
1 & 0 \\
0 & 1
\end{array}\right)\right)=p\left(\left(\begin{array}{cc}
-1 & 0 \\
0 & -1
\end{array}\right),\left(\begin{array}{cc}
-1 & 0 \\
0 & -1
\end{array}\right)\right),\right. \\
& p\left(\left(\begin{array}{cc}
\sqrt{-1} & 0 \\
0 & -\sqrt{-1}
\end{array}\right),\left(\begin{array}{cc}
\sqrt{-1} & 0 \\
0 & -\sqrt{-1}
\end{array}\right)\right)=p\left(\left(\begin{array}{cc}
-\sqrt{-1} & 0 \\
0 & \sqrt{-1}
\end{array}\right),\left(\begin{array}{cc}
-\sqrt{-1} & 0 \\
0 & \sqrt{-1}
\end{array}\right)\right), \\
& p\left(\left(\begin{array}{cc}
0 & -1 \\
1 & 0
\end{array}\right),\left(\begin{array}{cc}
0 & -1 \\
1 & 0
\end{array}\right)\right)=p\left(\left(\begin{array}{cc}
0 & 1 \\
-1 & 0
\end{array}\right),\left(\begin{array}{cc}
0 & 1 \\
-1 & 0
\end{array}\right)\right), \\
& \left.p\left(\left(\begin{array}{cc}
0 & \sqrt{-1} \\
\sqrt{-1} & 0
\end{array}\right),\left(\begin{array}{cc}
0 & \sqrt{-1} \\
\sqrt{-1} & 0
\end{array}\right)\right)=p\left(\left(\begin{array}{cc}
0 & -\sqrt{-1} \\
-\sqrt{-1} & 0
\end{array}\right),\left(\begin{array}{cc}
0 & -\sqrt{-1} \\
-\sqrt{-1} & 0
\end{array}\right)\right)\right\} \\
\cong & \mathbf{Z}_{2}+\mathbf{Z}_{2} .
\end{aligned}
$$

Hence the order of group $K_{0}$ is equal to 4 and

$$
\widetilde{K} / \widetilde{K}_{0} \cong K / K_{0}=S O(4) / \mathbf{Z}_{2}+\mathbf{Z}_{2} .
$$


For each $l, m \in \mathbf{Z}$ with $l, m \geq 0$, the vector subspace of $V_{l} \otimes V_{m}$

$\left(V_{l} \otimes V_{m}\right)_{\widetilde{K}_{0}}$

$$
:=\left\{\xi \in V_{l} \otimes V_{m} \mid\left[\left(\rho_{l} \otimes \rho_{m}\right)(A, B)\right](\xi)=\xi \text { for any }(A, B) \in \widetilde{K}_{0}\right\}
$$

can be described explicitly as follows:

LEMMA 4.4. When $(l+m) / 2$ is even,

$$
\left(V_{l} \otimes V_{m}\right)_{\tilde{K}_{0}}=\left\{\xi=\sum_{i+a: \text { even }} \xi_{i, a}\left(v_{i}^{(l)} \otimes v_{a}^{(m)}+v_{l-i}^{(l)} \otimes v_{m-a}^{(m)}\right) \mid \xi_{i, a} \in \mathbf{C}\right\}
$$

and when $(l+m) / 2$ is odd,

$$
\left(V_{l} \otimes V_{m}\right)_{\tilde{K}_{0}}=\left\{\xi=\sum_{i+a: o d d} \xi_{i, a}\left(v_{i}^{(l)} \otimes v_{a}^{(m)}-v_{l-i}^{(l)} \otimes v_{m-a}^{(m)}\right) \mid \xi_{i, a} \in \mathbf{C}\right\} .
$$

Next we describe the subgroups of $\widetilde{K}$ defined as

$$
\begin{aligned}
\widetilde{K}_{\mathfrak{a}} & :=\left\{(A, B) \in \widetilde{K} \mid\left[\left(\rho_{3} \otimes \rho_{1}\right)(A, B)\right](\mathfrak{a})=\mathfrak{a}\right\}, \\
\widetilde{K}_{[\mathfrak{a}]} & :=\left\{(A, B) \in \widetilde{K} \mid\left[\left(\rho_{3} \otimes \rho_{1}\right)(A, B)\right](\mathfrak{a})=\mathfrak{a}\right.
\end{aligned}
$$

$$
\text { preserving the orientation of } \mathfrak{a}\} \subset \widetilde{K}_{\mathfrak{a}} \text {. }
$$

For $(A, B) \in \widetilde{K}=S U(2) \times S U(2)$, we compute that $(A, B) \in \widetilde{K}_{\mathfrak{a}}$ if and only if $(A, B)$ is one of the following elements:

$$
\left(\left(\begin{array}{cc}
e^{\sqrt{-1} \theta_{1}} & 0 \\
0 & e^{-\sqrt{-1} \theta_{1}}
\end{array}\right),\left(\begin{array}{cc}
e^{\sqrt{-1} \theta_{1}^{\prime}} & 0 \\
0 & e^{-\sqrt{-1} \theta_{1}^{\prime}}
\end{array}\right)\right)
$$

where $\theta_{1}=\frac{\pi}{4} k_{1}, \theta_{1}^{\prime}=\frac{\pi}{4} k_{1}^{\prime}, k_{1}, k_{1}^{\prime} \in \mathbf{Z}, k_{1}-k_{1}^{\prime} \in 4 \mathbf{Z}$,

$$
\left(\left(\begin{array}{cc}
0 & -e^{-\sqrt{-1} \theta_{2}} \\
e^{\sqrt{-1} \theta_{2}} & 0
\end{array}\right),\left(\begin{array}{cc}
0 & -e^{-\sqrt{-1} \theta_{2}^{\prime}} \\
e^{\sqrt{-1} \theta_{2}^{\prime}} & 0
\end{array}\right)\right),
$$

where $\theta_{2}=\frac{\pi}{4} k_{2}, \theta_{2}^{\prime}=\frac{\pi}{4} k_{2}^{\prime}, k_{2}, k_{2}^{\prime} \in \mathbf{Z}, k_{2}-k_{2}^{\prime} \in 4 \mathbf{Z}$,

$$
\left(\left(\begin{array}{cc}
\frac{1}{\sqrt{2}} e^{\sqrt{-1} \theta_{1}} & -\frac{1}{\sqrt{2}} e^{-\sqrt{-1} \theta_{2}} \\
\frac{1}{\sqrt{2}} e^{\sqrt{-1} \theta_{2}} & \frac{1}{\sqrt{2}} e^{-\sqrt{-1} \theta_{1}}
\end{array}\right),\left(\begin{array}{cc}
\frac{1}{\sqrt{2}} e^{\sqrt{-1} \theta_{1}^{\prime}} & -\frac{1}{\sqrt{2}} e^{-\sqrt{-1} \theta_{2}^{\prime}} \\
\frac{1}{\sqrt{2}} e^{\sqrt{-1} \theta_{2}^{\prime}} & \frac{1}{\sqrt{2}} e^{-\sqrt{-1} \theta_{1}^{\prime}}
\end{array}\right)\right)
$$

where $\theta_{1}=\frac{\pi}{4} k_{1}, \theta_{2}=\frac{\pi}{4} k_{2}, \theta_{1}^{\prime}=\frac{\pi}{4} k_{1}^{\prime}, \theta_{2}^{\prime}=\frac{\pi}{4} k_{2}^{\prime}$ and $k_{1}, k_{2}, k_{1}^{\prime}, k_{2}^{\prime} \in \mathbf{Z}, k_{1}+k_{2}, k_{1}-k_{2}$, $k_{1}^{\prime}+k_{2}^{\prime}, k_{1}^{\prime}-k_{2}^{\prime} \in 2 \mathbf{Z}, k_{1}-k_{1}^{\prime}, k_{2}-k_{2}^{\prime} \in 4 \mathbf{Z}, k_{1}+k_{2}-k_{1}^{\prime}-k_{2}^{\prime}, k_{1}-k_{2}-k_{1}^{\prime}+k_{2}^{\prime} \in 8 \mathbf{Z}$.

In particular, the order of $\widetilde{K}_{\mathfrak{a}}$ is equal to $16+16+32+32=96$.

Moreover, for $(A, B) \in \widetilde{K}=S U(2) \times S U(2)$, we have that $(A, B) \in \widetilde{K}_{[\mathfrak{a}]}$ if and only if $(A, B)$ is one of the following elements:

$$
\left(\left(\begin{array}{cc}
e^{\sqrt{-1} \theta_{1}} & 0 \\
0 & e^{-\sqrt{-1} \theta_{1}}
\end{array}\right),\left(\begin{array}{cc}
e^{\sqrt{-1} \theta_{1}^{\prime}} & 0 \\
0 & e^{-\sqrt{-1} \theta_{1}^{\prime}}
\end{array}\right)\right)
$$


where $\theta_{1}=\frac{\pi}{4} k_{1}, \theta_{1}^{\prime}=\frac{\pi}{4} k_{1}^{\prime}, k_{1}, k_{1}^{\prime} \in 2 \mathbf{Z}, k_{1}-k_{1}^{\prime} \in 4 \mathbf{Z}$,

$$
\left(\left(\begin{array}{cc}
0 & -e^{-\sqrt{-1} \theta_{2}} \\
e^{\sqrt{-1} \theta_{2}} & 0
\end{array}\right),\left(\begin{array}{cc}
0 & -e^{-\sqrt{-1} \theta_{2}^{\prime}} \\
e^{\sqrt{-1} \theta_{2}^{\prime}} & 0
\end{array}\right)\right)
$$

where $\theta_{2}=\frac{\pi}{4} k_{2}, \theta_{2}^{\prime}=\frac{\pi}{4} k_{2}^{\prime}, k_{2}, k_{2}^{\prime} \in 2 \mathbf{Z}, k_{2}-k_{2}^{\prime} \in 4 \mathbf{Z}$,

$$
\left(\left(\begin{array}{cc}
\frac{1}{\sqrt{2}} e^{\sqrt{-1} \theta_{1}} & -\frac{1}{\sqrt{2}} e^{-\sqrt{-1} \theta_{2}} \\
\frac{1}{\sqrt{2}} e^{\sqrt{-1} \theta_{2}} & \frac{1}{\sqrt{2}} e^{-\sqrt{-1} \theta_{1}}
\end{array}\right),\left(\begin{array}{cc}
\frac{1}{\sqrt{2}} e^{\sqrt{-1} \theta_{1}^{\prime}} & -\frac{1}{\sqrt{2}} e^{-\sqrt{-1} \theta_{2}^{\prime}} \\
\frac{1}{\sqrt{2}} e^{\sqrt{-1} \theta_{2}^{\prime}} & \frac{1}{\sqrt{2}} e^{-\sqrt{-1} \theta_{1}^{\prime}}
\end{array}\right)\right)
$$

where $\theta_{1}=\frac{\pi}{4} k_{1}, \theta_{2}=\frac{\pi}{4} k_{2}, \theta_{1}^{\prime}=\frac{\pi}{4} k_{1}^{\prime}, \theta_{2}^{\prime}=\frac{\pi}{4} k_{2}^{\prime}$ and $k_{1}, k_{2}, k_{1}^{\prime}, k_{2}^{\prime} \in 2 \mathbf{Z}+1, k_{1}+k_{2}, k_{1}-k_{2}$, $k_{1}^{\prime}+k_{2}^{\prime}, k_{1}^{\prime}-k_{2}^{\prime} \in 2 \mathbf{Z}, k_{1}-k_{1}^{\prime}, k_{2}-k_{2}^{\prime} \in 4 \mathbf{Z}, k_{1}+k_{2}-\left(k_{1}^{\prime}+k_{2}^{\prime}\right), k_{1}-k_{2}-\left(k_{1}^{\prime}-k_{2}^{\prime}\right) \in 8 \mathbf{Z}$. In other words, $(A, B) \in \widetilde{K}_{[\mathfrak{a}]}$ if and only if $(A, B)$ is one of the following elements:

$$
\left(\left(\begin{array}{cc}
e^{\sqrt{-1} \theta_{1}} & 0 \\
0 & e^{-\sqrt{-1} \theta_{1}}
\end{array}\right),\left(\begin{array}{cc}
e^{\sqrt{-1} \theta_{1}^{\prime}} & 0 \\
0 & e^{-\sqrt{-1} \theta_{1}^{\prime}}
\end{array}\right)\right)
$$

where $\theta_{1}=\frac{\pi}{2} l_{1}, \theta_{1}^{\prime}=\frac{\pi}{2} l_{1}^{\prime}, l_{1}, l_{1}^{\prime} \in \mathbf{Z}, l_{1}-l_{1}^{\prime} \in 2 \mathbf{Z}$,

$$
\left(\left(\begin{array}{cc}
0 & -e^{-\sqrt{-1} \theta_{2}} \\
e^{\sqrt{-1} \theta_{2}} & 0
\end{array}\right),\left(\begin{array}{cc}
0 & -e^{-\sqrt{-1} \theta_{2}^{\prime}} \\
e^{\sqrt{-1} \theta_{2}^{\prime}} & 0
\end{array}\right)\right),
$$

where $\theta_{2}=\frac{\pi}{2} l_{2}, \theta_{2}^{\prime}=\frac{\pi}{2} l_{2}^{\prime}, l_{2}, l_{2}^{\prime} \in \mathbf{Z}, l_{2}-l_{2}^{\prime} \in 2 \mathbf{Z}$,

$$
\left(\left(\begin{array}{ll}
\frac{1}{\sqrt{2}} e^{\sqrt{-1} \theta_{1}} & -\frac{1}{\sqrt{2}} e^{-\sqrt{-1} \theta_{2}} \\
\frac{1}{\sqrt{2}} e^{\sqrt{-1} \theta_{2}} & \frac{1}{\sqrt{2}} e^{-\sqrt{-1} \theta_{1}}
\end{array}\right),\left(\begin{array}{cc}
\frac{1}{\sqrt{2}} e^{\sqrt{-1} \theta_{1}^{\prime}} & -\frac{1}{\sqrt{2}} e^{-\sqrt{-1} \theta_{2}^{\prime}} \\
\frac{1}{\sqrt{2}} e^{\sqrt{-1} \theta_{2}^{\prime}} & \frac{1}{\sqrt{2}} e^{-\sqrt{-1} \theta_{1}^{\prime}}
\end{array}\right)\right)
$$

where $\theta_{1}=\frac{\pi}{2} l_{1}+\frac{\pi}{4}, \theta_{2}=\frac{\pi}{2} l_{2}+\frac{\pi}{4}, \theta_{1}^{\prime}=\frac{\pi}{2} l_{1}^{\prime}+\frac{\pi}{4}, \theta_{2}^{\prime}=\frac{\pi}{2} l_{2}^{\prime}+\frac{\pi}{4}, l_{1}, l_{2}, l_{1}^{\prime}, l_{2}^{\prime} \in \mathbf{Z}$, $l_{1}-l_{1}^{\prime}, l_{2}-l_{2}^{\prime} \in 2 \mathbf{Z}, l_{1}+l_{2}-\left(l_{1}^{\prime}+l_{2}^{\prime}\right), l_{1}-l_{2}-\left(l_{1}^{\prime}-l_{2}^{\prime}\right) \in 4 \mathbf{Z}$. In particular, the order of $\widetilde{K}_{[\mathfrak{a}]}$ is equal to $8+8+16+16=48=8 \times 6=\sharp \widetilde{K}_{0} \times \sharp \mathbf{Z}_{6}$. Then we obtain

LEMMA 4.5. $\widetilde{K}_{[\mathfrak{a}]} / \widetilde{K}_{0} \cong \mathbf{Z}_{6}$.

ProOF. We compute

$$
\begin{aligned}
A & =\left(\begin{array}{cc}
\frac{1}{\sqrt{2}} e^{\sqrt{-1}\left(\frac{\pi}{2} l_{1}+\frac{\pi}{4}\right)} & -\frac{1}{\sqrt{2}} e^{-\sqrt{-1}\left(\frac{\pi}{2} l_{2}+\frac{\pi}{4}\right)} \\
\frac{1}{\sqrt{2}} e^{\sqrt{-1}\left(\frac{\pi}{2} l_{2}+\frac{\pi}{4}\right)} & \frac{1}{\sqrt{2}} e^{-\sqrt{-1}\left(\frac{\pi}{2} l_{1}+\frac{\pi}{4}\right)}
\end{array}\right), \\
A^{3} & =\left(\begin{array}{cc}
-\sqrt{2} \cos \left(\frac{\pi}{2} l_{1}+\frac{\pi}{4}\right) & 0 \\
0 & -\sqrt{2} \cos \left(\frac{\pi}{2} l_{1}+\frac{\pi}{4}\right)
\end{array}\right) \\
& =\left\{\begin{array}{lll}
-\mathrm{I}_{2} & \text { if } l_{1} \equiv 0 \text { or } 3(\bmod 4) \\
\mathrm{I}_{2} & \text { if } l_{1} \equiv 1 \text { or } 2(\bmod 4)
\end{array},\right. \\
A^{6} & =\mathrm{I}_{2} .
\end{aligned}
$$


TABLE 4. Small eigenvalues of $\mathcal{C}_{L}$ on $\tilde{K} / \tilde{K}_{0}$.

\begin{tabular}{|c|c|c|c|}
\hline$(l, m)$ & $\operatorname{dim}\left(V_{l} \otimes V_{m}\right)_{K_{0}}$ & eigenvalues of $\mathcal{C}_{L}$ & $-\lambda \leq 6$ \\
\hline$(1,1)$ & 1 & -3 & $*$ \\
\hline$(2,0)$ & 0 & & \\
\hline$(0,2)$ & 0 & $-3,-3$ & $*$ \\
\hline$(3,1)$ & 2 & $-9,-9$ & \\
\hline$(1,3)$ & 2 & $-3,-3$ & $*$ \\
\hline$(4,0)$ & 2 & $-15,-15$ & $*$ \\
\hline$(0,4)$ & 2 & $-5,-5,-8$ & $*$ \\
\hline$(2,2)$ & 3 & $-6,-8$ & $*$ \\
\hline$(5,1)$ & 3 & $-6,-9,-9$ & $*$ \\
\hline$(6,0)$ & 1 & $-12,-12,-12,-15$ & \\
\hline$(4,2)$ & 3 & $-10,-10$ & \\
\hline$(3,3)$ & 4 & & \\
\hline$(8,0)$ & 2 & & \\
\hline$(7,1)$ & 4 & & \\
\hline$(6,2)$ & 5 & & \\
\hline
\end{tabular}

The generator of $\widetilde{K}_{[\mathfrak{a}]} / \widetilde{K}_{0} \cong \mathbf{Z}_{6}$ is represented by the element

$$
\left(\left(\begin{array}{cc}
\frac{1+\sqrt{-1}}{2} & -\frac{1-\sqrt{-1}}{2} \\
\frac{1+\sqrt{-1}}{2} & \frac{1-\sqrt{-1}}{2}
\end{array}\right),\left(\begin{array}{cc}
-\frac{1+\sqrt{-1}}{2} & \frac{1-\sqrt{-1}}{2} \\
-\frac{1+\sqrt{-1}}{2} & -\frac{1-\sqrt{-1}}{2}
\end{array}\right)\right) .
$$

Then using Lemmas 4.3 and 4.4 we can determine directly all eigenvalues of $\mathcal{C}_{L}$ on $\tilde{K} / \tilde{K}_{0}$ less than or equal to $\operatorname{dim} L=6$ and corresponding representations of $\tilde{K}$ as in Table 4 . Hence we get

$$
\begin{aligned}
& \left\{(l, m) \mid-c_{L} \leq 6 \text { and }\left(V_{l} \otimes V_{m}\right)_{\tilde{K}_{0}} \neq\{0\}\right\} \\
= & \{(1,1),(4,0),(2,2),(3,1),(6,0),(5,1),(4,2)\} .
\end{aligned}
$$

Using the generator (16) of $\widetilde{K}_{[\mathfrak{a}]} / \widetilde{K}_{0} \cong \mathbf{Z}_{6}$, we compute that $\left(V_{l} \otimes V_{m}\right)_{\tilde{K}_{[\mathfrak{a}]}}=\{0\}$ for $(l, m)=(1,1),(4,0),(3,1),(5,1)$ and $\operatorname{dim}_{\mathbf{C}}\left(V_{l} \otimes V_{m}\right)_{\tilde{K}_{[\mathfrak{a}]}}=1$ for $(l, m)=(2,2),(6,0),(4,2)$. But we observe that the fixed vector in $\left(V_{2} \otimes V_{2}\right)_{\tilde{K}_{[\mathfrak{a}]}} \neq\{0\}$ corresponds to the larger eigenvalue $8>6$. Hence we obtain that the Gauss image $L^{6}=\mathcal{G}\left(\frac{S O(4)}{\mathbf{Z}_{2}+\mathbf{Z}_{2}}\right)=\frac{S O(4)}{\left(\mathbf{Z}_{2}+\mathbf{Z}_{2}\right) \cdot \mathbf{Z}_{6}} \subset Q_{6}(\mathbf{C})$ is Hamiltonian stable. 
Moreover from the above dimension computation we have

$$
\begin{aligned}
n\left(L^{6}\right) & =\operatorname{dim}_{\mathbf{C}} V_{6} \otimes V_{0}+\operatorname{dim}_{\mathbf{C}} V_{4} \otimes V_{2}=7 \times 1+5 \times 3=7+15=22 \\
& =\operatorname{dim} S O(8)-\operatorname{dim} S O(4)=n_{h k}(L) .
\end{aligned}
$$

Thus the Gauss image $L^{6}=\mathcal{G}\left(\frac{S O(4)}{\mathbf{Z}_{2}+\mathbf{Z}_{2}}\right)=\frac{S O(4)}{\left(\mathbf{Z}_{2}+\mathbf{Z}_{2}\right) \cdot \mathbf{Z}_{6}} \subset Q_{6}(\mathbf{C})$ is Hamiltonian rigid. From these results we conclude

THEOREM 4.6. The Gauss image $L^{6}=\mathcal{G}\left(\frac{S O(4)}{\mathbf{Z}_{2}+\mathbf{Z}_{2}}\right)=\frac{S O(4)}{\left(\mathbf{Z}_{2}+\mathbf{Z}_{2}\right) \cdot \mathbf{Z}_{6}} \subset Q_{6}(\mathbf{C})$ is strictly Hamiltonian stable.

5. The case $(U, K)=\left(E_{6}, U(1) \cdot \operatorname{Spin}(10)\right)$. Let $U=E_{6}$ and $K=U(1) \cdot \operatorname{Spin}(10)$. Then $(U, K)$ is of type $B C_{2}$. First we settle our notations for a symmetric space of type $E I I I$, following [21], [27], [10] and the references therein.

5.1. Cayley algebra. Let $\mathbf{K}$ be the real Cayley algebra. Let $\left\{c_{0}=1, c_{1}, \ldots, c_{7}\right\}$ be the standard basis of $\mathbf{K}$ satisfying the following relations ([21]):

$$
\begin{aligned}
& c_{i} c_{i+1}=-c_{i+1} c_{i}=c_{i+3}, \quad c_{i+1} c_{i+3}=-c_{i+3} c_{i+1}=c_{i}, \\
& c_{i+3} c_{i}=-c_{i} c_{i+3}=c_{i+1}, \quad c_{i}^{2}=-1 \text { for } i=1, \ldots, 7 .
\end{aligned}
$$

$\mathbf{K}$ is a noncommutative and nonassociative normed division algebra with the conjugation $x \mapsto$ $\bar{x}$ and the canonical inner product (, ) defined respectively by

$$
\overline{x_{0}+\sum_{i=1}^{7} x_{i} c_{i}}=x_{0}-\sum_{i=1}^{7} x_{i} c_{i}, \quad\left(\sum_{i=0}^{7} x_{i} c_{i}, \sum_{i=0}^{7} y_{i} c_{i}\right)=\sum_{i=0}^{7} x_{i} y_{i} .
$$

We extend the conjugation and the inner product $\mathbf{C}$-linearly to the complexified algebra $\mathbf{K}^{\mathbf{C}}$ of $\mathbf{K}$ and denote them by the same notions $x \mapsto \bar{x}$ and (, ) respectively. The automorphism group

$$
G_{2}:=\left\{\alpha \in \mathrm{GL}_{\mathbf{R}}(\mathbf{K}) \mid \alpha(x y)=\alpha(x) \alpha(y), \forall x, y \in \mathbf{K}\right\}
$$

of the Cayley algebra $\mathbf{K}$ is well-known to be a simply connected, connected compact Lie group of type $G_{2}$.

5.2. Exceptional Jordan algebra. The exceptional Jordan algebra $H_{3}(\mathbf{K})$ is defined as a real vector space

$$
H_{3}(\mathbf{K})=\left\{u \in M_{3}(\mathbf{K}) \mid \bar{u}^{t}=u\right\},
$$

equipped with the Jordan product

$$
u \circ v=\frac{1}{2}(u v+v u) \text { for } u, v \in H_{3}(\mathbf{K}) .
$$

$H_{3}(\mathbf{K})$ is of real dimension 27 and a typical element

$$
u=\left(\begin{array}{lll}
\xi_{1} & x_{3} & \bar{x}_{2} \\
\bar{x}_{3} & \xi_{2} & x_{1} \\
x_{2} & \bar{x}_{1} & \xi_{3}
\end{array}\right), \quad \xi_{i} \in \mathbf{R}, x_{i} \in \mathbf{K}
$$


of $H_{3}(\mathbf{K})$ is expressed as

$$
u=\xi_{1} e_{1}+\xi_{2} e_{2}+\xi_{3} e_{3}+x_{1} u_{1}+x_{2} u_{2}+x_{3} u_{3} .
$$

In $H_{3}(\mathbf{K})$, we define a trace $\operatorname{tr}(u)$ and an inner product $(u, v)$ respectively by

$$
\operatorname{tr}(u)=\xi_{1}+\xi_{2}+\xi_{3}, \quad(u, v):=\operatorname{tr}(u \circ v)
$$

for each $u, v \in H_{3}(\mathbf{K})$. Moreover, the Freudenthal product $u \times v$ is defined by

$$
u \times v:=\frac{1}{2}\left(2 u \circ v-\operatorname{tr}(u) v-\operatorname{tr}(v) u+(\operatorname{tr}(u) \operatorname{tr}(v)-(u, v)) \mathrm{I}_{3}\right),
$$

where $\mathrm{I}_{3}$ is the identity matrix of degree 3 , and a trilinear form $(u, v, w)$ and the determinant $\operatorname{det} u$ are defined respectively by

$$
(u, v, w)=(u, v \times w), \quad \operatorname{det} u=\frac{1}{3}(u, u, u) .
$$

Set

$$
S H_{3}(\mathbf{K})=\left\{u \in M_{3}(\mathbf{K}) \mid \bar{u}^{t}=-u, \operatorname{tr}(u)=0\right\} .
$$

Each element $u \in \mathrm{SH}_{3}(\mathbf{K})$ of the form

$$
u=\left(\begin{array}{ccc}
z_{1} & x_{3} & -\bar{x}_{2} \\
-\bar{x}_{3} & z_{2} & x_{1} \\
x_{2} & -\bar{x}_{1} & z_{3}
\end{array}\right), \quad z_{i}, x_{i} \in \mathbf{K}, \bar{z}_{i}=-z_{i}, \Sigma z_{i}=0
$$

is expressed as

$$
u=z_{1} e_{1}+z_{2} e_{2}+z_{3} e_{3}+x_{1} \bar{u}_{1}+x_{2} \bar{u}_{2}+x_{3} \bar{u}_{3} .
$$

Now we define two injective linear maps $R: H_{3}(\mathbf{K}) \rightarrow \mathfrak{g l}\left(H_{3}(\mathbf{K})\right)$ and $D: S H_{3}(\mathbf{K}) \rightarrow$ $\mathfrak{g l}\left(H_{3}(\mathbf{K})\right)$ by

$$
\begin{aligned}
& R(u) v=u \circ v=\frac{1}{2}(u v+v u) \quad \text { for } \quad u, v \in H_{3}(\mathbf{K}), \\
& D(u) v=\frac{1}{2}[u, v]=\frac{1}{2}(u v-v u) \quad \text { for } \quad u \in S H_{3}(\mathbf{K}), v \in H_{3}(\mathbf{K}) .
\end{aligned}
$$

Denote by $\mathfrak{D}$ and $\mathfrak{R}$ the images of $D$ and $R$ in $\mathfrak{g l}\left(H_{3}(\mathbf{K})\right)$, respectively. Introduce real vector subspaces of $\mathfrak{D}$ and $\mathfrak{R}$ as follows:

$$
\begin{aligned}
& \mathfrak{D}_{0}=\left\{\delta \in \mathfrak{D} \mid \delta\left(e_{i}\right)=0 \quad(i=1,2,3)\right\}, \\
& \mathfrak{D}_{i}=\left\{D\left(x \bar{u}_{i}\right) \mid x \in \mathbf{K}\right\} \text { for } i=1,2,3, \\
& \mathfrak{R}_{0}=\left\{R\left(\sum \xi_{i} e_{i}\right) \mid \xi_{i} \in \mathbf{R}, \sum \xi_{i}=0\right\}, \\
& \mathfrak{R}_{i}=\left\{R\left(x u_{i}\right) \mid x \in \mathbf{K}\right\} \text { for } i=1,2,3 .
\end{aligned}
$$

Remark that $\operatorname{dim} \mathfrak{D}_{0}=28, \operatorname{dim} \mathfrak{D}_{1}=\operatorname{dim} \mathfrak{D}_{2}=\operatorname{dim} \mathfrak{D}_{3}=8, \operatorname{dim} \mathfrak{R}_{0}=2$ and $\operatorname{dim} \mathfrak{R}_{1}=$ $\operatorname{dim} \mathfrak{R}_{2}=\operatorname{dim} \mathfrak{R}_{3}=8$. Moreover, we know that $\mathfrak{D}_{0}$ is a subalgebra of $\mathfrak{g l}\left(H_{3}(\mathbf{K})\right)$ generated by the set $\left\{D\left(\sum z_{i} e_{i}\right) \mid z_{i} \in \mathbf{K}, \bar{z}_{i}=-z_{i}, \sum z_{i}=0\right\}$. In fact, $\mathfrak{D}_{0}$ is isomorphic to the Lie algebra $\mathfrak{o}(8)$ and its basis can be chosen as $\left\{D_{i, r}(1 \leq r \leq 7), D_{i, p q}(1 \leq p<q \leq 7)\right\}$ for 
$i=1,2$ or 3 ([6], [10], [27]). We now explain in details by using Ise's notions ([10, p.82]). Set

$$
D_{i, r}=D\left(c_{r}\left(-e_{j}+e_{k}\right)\right), \quad(1 \leq i \leq 3,1 \leq r \leq 7)
$$

and

$$
D_{i, p q}=\left[D_{i, p}, D_{i, q}\right], \quad(1 \leq i \leq 3,1 \leq p, q \leq 7),
$$

where $\{i, j, k\}$ is a cyclic permutation of $\{1,2,3\}$. Then from

$$
D\left(\sum_{i=1}^{3} z_{i} e_{i}\right)(v)=\frac{1}{2} \sum_{\{i, j, k\}}\left(z_{j} x_{i}-x_{i} z_{k}\right) u_{i},
$$

we obtain

$$
\left\{\begin{array}{l}
D_{i, r}\left(x u_{i}\right)= \begin{cases}-c_{r} u_{i} & \text { if } x=c_{0} \\
c_{0} u_{i} & \text { if } x=c_{r} \\
0 & \text { if } x=c_{q}(q \neq r)\end{cases} \\
D_{i, r}\left(x u_{j}\right)=\frac{1}{2}\left(c_{r} x\right) u_{j}, \\
D_{i, r}\left(x u_{k}\right)=\frac{1}{2}\left(x c_{r}\right) u_{k},
\end{array}\right.
$$

and

$$
\left\{\begin{array}{l}
D_{i, p q}\left(x u_{i}\right)=\left\{\begin{array}{ll}
c_{q} u_{i} & \text { if } x=c_{p} \\
-c_{p} u_{i} & \text { if } x=c_{q} \\
0 & \text { if } x=c_{r}
\end{array}(r \leq 0, \neq p, q),\right. \\
D_{i, p q}\left(x u_{j}\right)=\frac{1}{2}\left\{c_{p}\left(c_{q} x\right)\right\} u_{j}, \\
D_{i, p q}\left(x u_{k}\right)=\frac{1}{2}\left\{\left(x c_{q}\right) c_{p}\right\} u_{k} .
\end{array}\right.
$$

These mean that every $D_{i, r}, D_{i, p q}$ leave $\mathfrak{T}_{i}=\left\{x u_{i} \mid x \in \mathbf{K}\right\}$ invariant $(1 \leq i \leq 3,1 \leq$ $p, q, r \leq 7)$ and identifying $\mathfrak{T}_{i}$ with $\mathbf{K}$, it is represented as a skew-symmetric matrix with respect to the basis $\left\{c_{0}, c_{1}, \ldots, c_{7}\right\}$, that is, $D_{i, r}=E_{0 r}-E_{r 0}$ and $D_{i, p q}=E_{q p}-E_{p q}$, where $E_{p q}$ denotes the $8 \times 8$ matrix with all 0-components except $(p, q)$-component, 1 . Moreover,

$$
\begin{aligned}
{\left[D_{i, r}, D_{i, p q}\right] } & =D_{i, p} \delta_{q r}-D_{i, q} \delta_{r p}, \\
{\left[D_{i, p q}, D_{i, r s}\right] } & =D_{i, p r} \delta_{s q}+D_{i, q s} \delta_{p r}+D_{i, r q} \delta_{s p}+D_{i, s p} \delta_{r q},
\end{aligned}
$$

where $1 \leq i \leq 3$ and $1 \leq p, q, r, s \leq 7$. Particularly, we have

$$
\left[D_{i, r}, D_{i, p q}\right]=0, \quad\left[D_{i, p q}, D_{i, r s}\right]=0,
$$

if $p, q, r, s$ are all different from each other. Denote by $\mathfrak{D}_{i, 0}$ a real linear space spanned by all $D_{i, r}, D_{i, p q}(1 \leq p, q, r \leq 7)$. Then all $\mathfrak{D}_{i, 0}(1 \leq i \leq 3)$ are isomorphic to each other, and they are isomorphic to the Lie algebra $\mathfrak{o}(8)$. We shall use $\mathfrak{D}_{0}=\mathfrak{D}_{1,0}$ in the next argument.

Let

$$
H_{3}(\mathbf{K}) \mathbf{C}:=H_{3}(\mathbf{K})+\sqrt{-1} H_{3}(\mathbf{K})
$$


be the complexification of $H_{3}(\mathbf{K})$. Then there are two complex conjugations on $H_{3}(\mathbf{K})$, namely,

$$
\overline{u_{1}+\sqrt{-1} u_{2}}=\bar{u}_{1}+\sqrt{-1} \bar{u}_{2}, \quad \tau\left(u_{1}+\sqrt{-1} u_{2}\right)=u_{1}-\sqrt{-1} u_{2},
$$

where $u_{1}, u_{2} \in H_{3}(\mathbf{K})$. Then $H_{3}(\mathbf{K}){ }^{\mathbf{C}}$ is canonically identified with

$$
H_{3}\left(\mathbf{K}^{\mathbf{C}}\right)=\left\{u \in M_{3}\left(\mathbf{K}^{\mathbf{C}}\right) \mid \bar{u}^{t}=u\right\} .
$$

An element $u \in H_{3}\left(\mathbf{K}^{\mathbf{C}}\right)$ of the form (17), with $\xi_{i} \in \mathbf{C}, x_{i} \in \mathbf{K}^{\mathbf{C}}$, is also expressed as $u=\xi_{1} e_{1}+\xi_{2} e_{2}+\xi_{3} e_{3}+x_{1} u_{1}+x_{2} u_{2}+x_{3} u_{3}$. The standard Hermitian inner product $\langle$, of $H_{3}\left(\mathbf{K}^{\mathbf{C}}\right)$ is defined by $\langle u, v\rangle:=(\tau u, v)$. Meanwhile, the complexification $\mathrm{SH}_{3}(\mathbf{K}){ }^{\mathbf{C}}$ of $\mathrm{SH}_{3}(\mathbf{K})$ is identified with

$$
S H_{3}\left(\mathbf{K}^{\mathbf{C}}\right)=\left\{u \in M_{3}\left(\mathbf{K}^{\mathbf{C}}\right) \mid \bar{u}^{t}=-u, \operatorname{tr}(u)=0\right\},
$$

whose element $u$ of the form (18), with $z_{i}, x_{i} \in \mathbf{K}^{\mathbf{C}}$, is also expressed as $u=z_{1} e_{1}+z_{2} e_{2}+$ $z_{3} e_{3}+x_{1} \bar{u}_{1}+x_{2} \bar{u}_{2}+x_{3} \bar{u}_{3}$. Then $D(u) \in \mathfrak{g l}\left(H_{3}\left(\mathbf{K}^{\mathbf{C}}\right)\right)$ for $u \in S H_{3}\left(\mathbf{K}^{\mathbf{C}}\right)$ and $R(u) \in$ $\mathfrak{g l}\left(H_{3}\left(\mathbf{K}^{\mathbf{C}}\right)\right)$ for $u \in H_{3}\left(\mathbf{K}^{\mathbf{C}}\right)$ can be defined in the same way as (19).

5.3. The groups $F_{4}$ and $E_{6}$. We shall use the setup in [27] and [10]. The automorphism group of the Jordan algebra $H_{3}(\mathbf{K})$

$$
\begin{aligned}
F_{4}: & =\left\{\alpha \in G L\left(H_{3}(\mathbf{K})\right) \mid \alpha(u \circ v)=\alpha u \circ \alpha v\right\} \\
& =\left\{\alpha \in G L\left(H_{3}(\mathbf{K})\right) \mid \operatorname{det}(\alpha u)=\operatorname{det} u,(\alpha u, \alpha v)=(u, v)\right\}
\end{aligned}
$$

is known to be a connected, simply connected, compact Lie group of type $F_{4}$. Its Lie algebra $\mathfrak{f}_{4}$ is thus given by

$$
\mathfrak{f}_{4}:=\left\{\delta \in \mathfrak{g l}\left(H_{3}(\mathbf{K})\right) \mid \delta(u \circ v)=\delta u \circ v+u \circ \delta v\right\},
$$

which is isomorphic to $\mathfrak{D}=\mathfrak{D}_{0}+\mathfrak{D}_{1}+\mathfrak{D}_{2}+\mathfrak{D}_{3}$. We are interested in the following two subgroups of $F_{4}$ :

$$
\begin{aligned}
\left(F_{4}\right)_{e_{1}} & :=\left\{\alpha \in F_{4} \mid \alpha e_{1}=e_{1}\right\} \cong \operatorname{Spin}(9), \\
\left(F_{4}\right)_{e_{1}, e_{2}, e_{3}} & :=\left\{\alpha \in F_{4} \mid \alpha e_{i}=e_{i}(i=1,2,3)\right\} \cong \operatorname{Spin}(8) .
\end{aligned}
$$

Note that the Lie algebra of $\left(F_{4}\right)_{e_{1}, e_{2}, e_{3}}$ is isomorphic to $\mathfrak{D}_{0}$.

The group $G_{2}$ can be realized as a subgroup of $F_{4}$. For each $\alpha \in G_{2}$, we define an R-linear transformation $\tilde{\alpha}$ of $H_{3}(\mathbf{K})$ by

$$
\tilde{\alpha}\left(\begin{array}{ccc}
\xi_{1} & x_{3} & \bar{x}_{2} \\
\bar{x}_{3} & \xi_{2} & x_{1} \\
x_{2} & \bar{x}_{1} & \xi_{3}
\end{array}\right):=\left(\begin{array}{ccc}
\xi_{1} & \alpha x_{3} & \overline{\alpha x_{2}} \\
\overline{\alpha x_{3}} & \xi_{2} & \alpha x_{1} \\
\alpha x_{2} & \overline{\alpha x_{1}} & \xi_{3}
\end{array}\right),
$$

for $x_{i} \in \mathbf{K}(i=1,2,3)$. Then $\tilde{\alpha} \in F_{4}$. Identifying $\alpha$ with $\tilde{\alpha}$, we consider $G_{2}$ as a subgroup of $F_{4}$.

The groups $E_{6}$ and $E_{6}^{\mathbf{C}}$ defined by

$$
\begin{aligned}
& E_{6}:=\left\{\alpha \in G L_{\mathbf{C}}\left(H_{3}(\mathbf{K}) \mathbf{C}^{\mathbf{C}}\right) \mid \operatorname{det}(\alpha u)=\operatorname{det} u,\langle\alpha u, \alpha v\rangle=\langle u, v\rangle\right\}, \\
& E_{6}^{\mathbf{C}}:=\left\{\alpha \in G L_{\mathbf{C}}\left(H_{3}(\mathbf{K}) \mathbf{C}^{\mathbf{C}}\right) \mid \operatorname{det}(\alpha u)=\operatorname{det}(u)\right\},
\end{aligned}
$$


are known to be a connected, simply connected, compact Lie group of type $E_{6}$ and its complexification, respectively. Hence $F_{4}$ is a compact subgroup of $E_{6}$. The Lie algebras of $E_{6}$ and $E_{6}^{\mathbf{C}}$ are given respectively by

$$
\begin{aligned}
& \mathfrak{e}_{6}:=\left\{\phi \in \mathfrak{g l}_{\mathbf{C}}\left(H_{3}(\mathbf{K})^{\mathbf{C}}\right) \mid(\phi u, u, u)=0,\langle\phi u, v\rangle+\langle u, \phi v\rangle=0\right\}, \\
& \mathfrak{e}_{6}^{\mathbf{C}}:=\left\{\phi \in \mathfrak{g l}_{\mathbf{C}}\left(H_{3}(\mathbf{K})^{\mathbf{C}}\right) \mid(\phi u, u, u)=0\right\} .
\end{aligned}
$$

We know ([27, p.68]) that any element $\phi \in \mathfrak{e}_{6}^{\mathbf{C}}$ is uniquely expressed as

$$
\phi=\delta+\varsigma, \quad \delta \in \mathfrak{D}^{\mathbf{C}}, \quad \varsigma \in \mathfrak{R}^{\mathbf{C}},
$$

where $\mathfrak{D}^{\mathbf{C}}$ and $\mathfrak{R}^{\mathbf{C}}$ denote the complexifications of $\mathfrak{D}$ and $\mathfrak{R}$ respectively. So we get the socalled Chevalley-Schafer model ([6]) of $\mathfrak{e}_{6}^{\mathbf{C}}: \mathfrak{e}_{6}^{\mathbf{C}}=\mathfrak{D}^{\mathbf{C}}+\mathfrak{R}^{\mathbf{C}}$ as a subalgebra of $\mathfrak{g l}\left(H_{3}(\mathbf{K}){ }^{\mathbf{C}}\right)$. The inclusion $\phi: \mathfrak{e}_{6}^{\mathbf{C}} \subset \mathfrak{g l}\left(H_{3}(\mathbf{K}){ }^{\mathbf{C}}\right)$ is a 27-dimensional irreducible representation of $\mathfrak{e}_{6}^{\mathbf{C}}$. Moreover, any element $\phi \in \mathfrak{e}_{6}$ is uniquely expressed as

$$
\phi=\delta+\sqrt{-1} \varsigma, \quad \delta \in \mathfrak{D}, \quad \varsigma \in \mathfrak{R} .
$$

Thus we have the direct sum decomposition $\mathfrak{e}_{6}=\mathfrak{D}+\sqrt{-1} \mathfrak{R}$.

\subsection{Basic formulas in $\mathfrak{e}_{6}$.}

LEMMA 5.1. For $v=\xi_{1} e_{1}+\xi_{2} e_{2}+\xi_{3} e_{3}+x_{1} u_{1}+x_{2} u_{2}+x_{3} u_{3} \in H_{3}\left(\mathbf{K}^{\mathbf{C}}\right)$, we have

$$
\begin{aligned}
R\left(\sum \eta_{l} e_{l}\right) v= & \eta_{1} \xi_{1} e_{1}+\eta_{2} \xi_{2} e_{2}+\eta_{3} \xi_{3} e_{3}+\frac{1}{2}\left(\eta_{2}+\eta_{3}\right) x_{1} u_{1} \\
& +\frac{1}{2}\left(\eta_{3}+\eta_{1}\right) x_{2} u_{2}+\frac{1}{2}\left(\eta_{1}+\eta_{2}\right) x_{3} u_{3}, \\
D\left(\sum z_{l} e_{l}\right) v= & \frac{1}{2}\left(z_{2} x_{1}-x_{1} z_{3}\right) u_{1}+\frac{1}{2}\left(z_{3} x_{2}-x_{2} z_{1}\right) u_{2}+\frac{1}{2}\left(z_{1} x_{3}-x_{3} z_{2}\right) u_{3}, \\
D\left(x \bar{u}_{i}\right) v= & \left(x, x_{i}\right)\left(e_{j}-e_{k}\right)+\frac{1}{2}\left(\xi_{k}-\xi_{j}\right) x u_{i}-\frac{1}{2}\left(\bar{x} \bar{x}_{k}\right) u_{j}+\frac{1}{2}\left(\bar{x}_{j} \bar{x}\right) u_{k}, \\
R\left(x u_{i}\right) v= & \left(x, x_{i}\right)\left(e_{j}+e_{k}\right)+\frac{1}{2}\left(\xi_{j}+\xi_{k}\right) x u_{i}+\frac{1}{2} \bar{x} \bar{x}_{k} u_{j}+\frac{1}{2} \bar{x}_{j} \bar{x} u_{k},
\end{aligned}
$$

where $\{i, j, k\}$ is a cyclic permutation of $\{1,2,3\}$.

The relations (20), (21), (22) and the following list give commutation rules for $\mathfrak{e}_{6}^{\mathbf{C}}$. Here, $x, y, z_{i} \in \mathbf{K}^{\mathbf{C}}, \bar{z}_{i}=-z_{i}$ for $i=1,2,3, \sum_{i} z_{i}=0$, and $\xi_{1}, \xi_{2}, \xi_{3} \in \mathbf{C}$ with $\sum_{l} \xi_{l}=0$. In formulae (23) (31), $(i, j, k)$ is a cyclic permutation of $(1,2,3)$. In formulae (33) and (34), $i=1,2,3$.

$$
\begin{aligned}
& {\left[R\left(x u_{i}\right), R\left(y u_{j}\right)\right]=-(1 / 2) D\left(\overline{x y} \bar{u}_{k}\right),} \\
& {\left[R\left(x u_{i}\right), D\left(y u_{j}\right)\right]=\left[D\left(x \bar{u}_{i}\right), R\left(y u_{j}\right)\right]=(1 / 2) R\left(\overline{x y} \bar{u}_{k}\right),} \\
& {\left[D\left(x \bar{u}_{i}\right), D\left(y \bar{u}_{j}\right)\right]=-(1 / 2) D\left(\overline{x y} \bar{u}_{k}\right),} \\
& {\left[D\left(x \bar{u}_{i}\right), R\left(y \bar{u}_{i}\right)\right]=(x, y) R\left(e_{j}-e_{k}\right),} \\
& {\left[R\left(\sum \xi_{l} e_{l}\right), R\left(x \bar{u}_{i}\right)\right]=(1 / 2)\left(\xi_{j}-\xi_{k}\right) D\left(x \bar{u}_{i}\right),}
\end{aligned}
$$




$$
\begin{aligned}
& {\left[R\left(\sum \xi_{l} e_{l}\right), D\left(x \bar{u}_{i}\right)\right]=(1 / 2)\left(\xi_{j}-\xi_{k}\right) R\left(x \bar{u}_{i}\right),} \\
& {\left[D\left(\sum z_{l} e_{l}\right), D\left(x \bar{u}_{i}\right)\right]=(1 / 2) D\left(\left(z_{j} x-x z_{k}\right) \bar{u}_{i}\right),} \\
& {\left[D\left(\sum z_{l} e_{l}\right), R\left(x \bar{u}_{i}\right)\right]=(1 / 2) R\left(\left(z_{j} x-x z_{k}\right) u_{i}\right),} \\
& {\left[R\left(x u_{i}\right), R\left(y u_{i}\right)\right]=-\left[D\left(x \bar{u}_{i}\right), D\left(y \bar{u}_{i}\right)\right]} \\
& =D\left(\left(\frac{y+\bar{y}}{2} \frac{x-\bar{x}}{2}-\frac{x+\bar{x}}{2} \frac{y-\bar{y}}{2}\right)\left(e_{j}-e_{k}\right)\right) \\
& \quad-\left[D\left(\frac{x-\bar{x}}{2}\left(e_{j}-e_{k}\right)\right), D\left(\frac{y-\bar{y}}{2}\left(e_{j}-e_{k}\right)\right)\right] \\
& {\left[\mathfrak{R}_{0}^{\mathbf{C}}, \mathfrak{R}_{0}^{\mathbf{C}}+\mathfrak{D}_{0}^{\mathbf{C}}\right]=\{0\},} \\
& {\left[R\left(x u_{i}\right),\left[R\left(x u_{i}\right), R\left(y u_{i}\right)\right]\right]=R\left(((x, x) y-(x, y) x) u_{i}\right),} \\
& {\left[D\left(x \bar{u}_{i}\right),\left[D\left(x \bar{u}_{i}\right), D\left(y \bar{u}_{i}\right)\right]\right]=D\left(((x, y) x-(x, x) y) \bar{u}_{i}\right) .}
\end{aligned}
$$

We remark that the Killing-Cartan form $B$ of $\mathfrak{e}_{6}^{\mathbf{C}}$ is given by ([10, p.88] or [27, p.74])

$$
B(u, v)=4 \operatorname{tr}(u v),
$$

for each $u, v \in \mathfrak{e}_{6}^{\mathbf{C}} \subset \mathfrak{g l}\left(H_{3}(\mathbf{K}){ }^{\mathbf{C}}\right)$.

5.5. Realization of $E_{6} /(U(1) \cdot \operatorname{Spin}(10))$. Consider a $\mathbf{C}$-linear transformation $\sigma$ of $H_{3}(\mathbf{K})^{\mathbf{C}}$ defined by

$$
\sigma\left(\begin{array}{lll}
\xi_{1} & x_{3} & \bar{x}_{2} \\
\bar{x}_{3} & \xi_{2} & x_{1} \\
x_{2} & \bar{x}_{1} & \xi_{3}
\end{array}\right)=\left(\begin{array}{ccc}
\xi_{1} & -x_{3} & -\bar{x}_{2} \\
-\bar{x}_{3} & \xi_{2} & x_{1} \\
-x_{2} & \bar{x}_{1} & \xi_{3}
\end{array}\right)
$$

Then $\sigma \in E_{6}$ and $\sigma^{2}=1 . \sigma$ induces an involutive automorphism of $E_{6}$ by $\alpha \mapsto \sigma \alpha \sigma$, which is also denoted by $\sigma$. In order to investigate the subgroup $\left(E_{6}\right)^{\sigma}$ of all fixed elements by $\sigma$,

$$
\left(E_{6}\right)^{\sigma}=\left\{\alpha \in E_{6} \mid \sigma \alpha=\alpha\right\}
$$

consider two subgroups

$$
\left(E_{6}\right)_{e_{1}}=\left\{\alpha \in E_{6} \mid \alpha e_{1}=e_{1}\right\} \cong \operatorname{Spin}(10)
$$

and

$$
U(1)=\left\{\phi(\theta) \in G L_{\mathbf{C}}\left(H_{3}(\mathbf{K})^{\mathbf{C}}\right) \mid \theta=e^{\sqrt{-1} t / 2}, t \in \mathbf{R}\right\},
$$

where $\phi(\theta):=\exp \left(t \sqrt{-1} R\left(2 e_{1}-e_{2}-e_{3}\right)\right) \in G L_{\mathbf{C}}\left(H_{3}(\mathbf{K}) \mathbf{C}\right)$ and

$$
\phi(\theta)\left(\begin{array}{ccc}
\xi_{1} & x_{3} & \bar{x}_{2} \\
\bar{x}_{3} & \xi_{2} & x_{1} \\
x_{2} & \bar{x}_{1} & \xi_{3}
\end{array}\right)=\left(\begin{array}{ccc}
\theta^{4} \xi_{1} & \theta x_{3} & \theta \bar{x}_{2} \\
\theta \bar{x}_{3} & \theta^{-2} \xi_{2} & \theta^{-2} x_{1} \\
\theta x_{2} & \theta^{-2} \bar{x}_{1} & \theta^{-2} \xi_{3}
\end{array}\right) .
$$

Here the subgroups $U(1)$ and $\operatorname{Spin}(10)$ of $\left(E_{6}\right)^{\sigma}$ are elementwise commutative. Define a mapping

$$
p: \tilde{K}=U(1) \times \operatorname{Spin}(10) \ni(\theta, \alpha) \rightarrow \phi(\theta) \alpha \in K=(E)^{\sigma},
$$


which is a surjective Lie group homomorphism. Since

$$
U(1) \cap \operatorname{Spin}(10)=\{1=\phi(1), \phi(-1), \phi(\sqrt{-1}), \phi(-\sqrt{-1})\},
$$

we have $\operatorname{Ker}(p)=\{(1, \phi(1)),(-1, \phi(-1)),(\sqrt{-1}, \phi(-\sqrt{-1})),(-\sqrt{-1}, \phi(\sqrt{-1}))\}$, which is isomorphic to $\mathbf{Z}_{4}$. Thus

$$
K=\left(E_{6}\right)^{\sigma}=\tilde{K} / \mathbf{Z}_{4}=(U(1) \times \operatorname{Spin}(10)) / \mathbf{Z}_{4}=: U(1) \cdot \operatorname{Spin}(10),
$$

and $U / K=E_{6} /(U(1) \cdot \operatorname{Spin}(10))$. Correspondingly, we have

$$
\mathfrak{k}=\left(\mathfrak{e}_{6}\right)_{\sigma}=\left\{\phi \in \mathfrak{e}_{6} \mid \sigma_{*} \phi=\phi\right\}=\left(\mathfrak{e}_{6}\right)_{e_{1}}+\mathbf{R} \sqrt{-1} R\left(2 e_{1}-e_{2}-e_{3}\right) .
$$

Since for any $\phi \in \mathfrak{e}_{6}$ there exist $u \in S_{3}(\mathbf{K})$ and $v \in H_{3}(\mathbf{K})$ such that

$$
\phi e_{1}=D(u)\left(e_{1}\right)+\sqrt{-1} R(v)\left(e_{1}\right),
$$

it holds that $\phi e_{1}=0$ if and only if

$$
u=z_{1} e_{1}+z_{2} e_{2}+z_{3} e_{3}+a_{1} \bar{u}_{1} \in S H_{3}(\mathbf{K}), \quad v=\xi_{2} e_{2}+\xi_{3} e_{3}+x_{1} u_{1} \in H_{3}(\mathbf{K}),
$$

where $a_{1}, x_{1} \in \mathbf{K}, \xi_{2}, \xi_{3} \in \mathbf{R}$ with $\xi_{2}+\xi_{3}=0$. Hence

$$
\begin{aligned}
\left(\mathfrak{e}_{6}\right)_{e_{1}} & :=\left\{\phi \in \mathfrak{e}_{6} \mid \phi e_{1}=0\right\} \\
& =\mathfrak{D}_{0}+\mathfrak{D}_{1}+\mathbf{R} \sqrt{-1} R\left(e_{2}-e_{3}\right)+\sqrt{-1} \mathfrak{R}_{1} \cong \mathfrak{o}(10) .
\end{aligned}
$$

Therefore, the Cartan decomposition of a compact simple Lie algebra $\mathfrak{u}=\mathfrak{e}_{6}$ of type EIII is given as

$$
\begin{aligned}
& \mathfrak{u}=\mathfrak{e}_{6}=\mathfrak{D}+\sqrt{-1} \mathfrak{R}, \\
& \mathfrak{k}=\left(\mathfrak{e}_{6}\right)_{\sigma}=\mathfrak{D}_{0}+\mathfrak{D}_{1}+\sqrt{-1} \mathfrak{R}_{0}+\sqrt{-1} \mathfrak{R}_{1}, \\
& \mathfrak{p}=(\mathfrak{e})_{-\sigma}=\mathfrak{D}_{2}+\mathfrak{D}_{3}+\sqrt{-1} \mathfrak{R}_{2}+\sqrt{-1} \mathfrak{R}_{3},
\end{aligned}
$$

where $\mathfrak{k}$ is isomorphic to $\mathfrak{u}(1)+\mathfrak{o}(10)$,

$$
[\mathfrak{k}, \mathfrak{k}]=\mathfrak{D}_{0}+\mathfrak{D}_{1}+\sqrt{-1} \mathbf{R} R\left(e_{2}-e_{3}\right)+\sqrt{-1} \mathfrak{R}_{1}=\left(\mathfrak{e}_{6}\right)_{e_{1}}
$$

is isomorphic to $\mathfrak{o}(10)$ and the center of $\mathfrak{k}$ is spanned by

$$
Z=\sqrt{-1} R\left(2 e_{1}-e_{2}-e_{3}\right) .
$$

On the other hand, a compact Hermitian symmetric space of type EIII can be defined by ([1, p. 74-75])

$$
\mathrm{EIII}=\left\{u \in H_{3}(\mathbf{K})^{\mathbf{C}} \mid u \times u=0, u \neq 0\right\} / \mathbf{C}^{*} \subset P\left(H_{3}(\mathbf{K}){ }^{\mathbf{C}}\right),
$$

which is considered as a compact complex submanifold embedded in a complex projective space $\mathbf{C} P^{26}$. Since $E_{6}$ acts transitively on EIII and the isotropy subgroup of $E_{6}$ at $o=\left[e_{1}\right]$ is $\left(E_{6}\right)^{\sigma}$, we know that $\mathrm{EIII} \cong E_{6} /\left(E_{6}\right)^{\sigma}=E_{6} /(U(1) \cdot \operatorname{Spin}(10))$. Under the Hopf fibration $H_{3}(\mathbf{K})^{\mathbf{C}} \supset S^{53}(1) \ni e_{1} \mapsto o=\left[e_{1}\right] \in P\left(H_{3}(\mathbf{K})^{\mathbf{C}}\right)$, the tangent vector space $T_{o}(U / K)$ at $o$ is linearly isomorphic to a vector subspace

$$
\begin{aligned}
T_{o}(\mathrm{EIII}) & \cong\left\{u \in H_{3}(\mathbf{K}) \mathbf{C} \mid u \times e_{1}=0,\left\langle u, e_{1}\right\rangle=0\right\} \\
& =\left\{x_{2} u_{2}+x_{3} u_{3} \mid x_{2}, x_{3} \in \mathbf{K}^{\mathbf{C}}\right\} .
\end{aligned}
$$


The differential of the natural projection $p: U=E_{6} \rightarrow U / K=$ EIII induces a linear isomorphism $p_{*}: \mathfrak{p} \rightarrow T_{o}(\mathrm{EIII})$. Then $p_{*}(\phi)=\phi\left(e_{1}\right)$ and

$$
\begin{gathered}
p_{*}\left(2\left(D\left(x_{2} \bar{u}_{2}\right)-D\left(x_{3} \bar{u}_{3}\right)\right)+2 \sqrt{-1}\left(R\left(x_{2}^{\prime} u_{2}\right)+R\left(x_{3}^{\prime} u_{3}\right)\right)\right) \\
=\left(x_{2}+\sqrt{-1} x_{2}^{\prime}\right) u_{2}+\left(x_{3}+\sqrt{-1} x_{3}^{\prime}\right) u_{3} .
\end{gathered}
$$

5.6. Restricted root systems of EIII. Define $H_{1}, H_{2} \in \mathfrak{p}$ by

$$
\begin{aligned}
& H_{1}=D \bar{u}_{2}+\sqrt{-1} R\left(c_{4} u_{2}\right), \\
& H_{2}=D \bar{u}_{2}-\sqrt{-1} R\left(c_{4} u_{2}\right) .
\end{aligned}
$$

Then by (26), $\left[H_{1}, H_{2}\right]=0$. Hence

$$
\mathfrak{a}=\left\{H\left(\xi_{1}, \xi_{2}\right)=\xi_{1} H_{1}+\xi_{2} H_{2} \mid \xi_{1}, \xi_{2} \in \mathbf{R}\right\}
$$

is a maximal abelian subalgebra in $\mathfrak{p}$. We remark that this maximal abelian subalgebra $\mathfrak{a}$ is different from ones given by M. Ise and used in [21]. Then by direct computations using (20)-(34), we get the following restricted root system decomposition of $\mathfrak{k}$ and $\mathfrak{p}$ :

$$
\begin{aligned}
\mathfrak{k} & =\mathfrak{k}_{0}+\mathfrak{k}_{2 \xi_{1}}+\mathfrak{k}_{2 \xi_{2}}+\mathfrak{k}_{\xi_{1}+\xi_{2}}+\mathfrak{k}_{\xi_{1}-\xi_{2}}+\mathfrak{k}_{\xi_{1}}+\mathfrak{k}_{\xi_{2}}, \\
\mathfrak{p} & =\mathfrak{a}+\mathfrak{p}_{2 \xi_{1}}+\mathfrak{p}_{2 \xi_{2}}+\mathfrak{p}_{\xi_{1}+\xi_{2}}+\mathfrak{p}_{\xi_{1}-\xi_{2}}+\mathfrak{p}_{\xi_{1}}+\mathfrak{p}_{\xi_{2}},
\end{aligned}
$$

where

$$
\begin{aligned}
& \mathfrak{k}_{0}=\{X \in \mathfrak{k} \mid[X, H]=0 \quad \text { for each } H \in \mathfrak{a}\}, \\
& =\operatorname{span}_{\mathbf{R}}\left\{\sqrt{-1} R\left(e_{1}-2 e_{2}+e_{3}\right)\right\}+\operatorname{span}_{\mathbf{R}}\left\{-D_{1,4}+D_{1,12}, D_{1,12}+D_{1,36},\right. \\
& D_{1,36}+D_{1,57},-D_{1,1}+D_{1,24},-D_{1,2}-D_{1,14},-D_{1,3}+D_{1,46},-D_{1,5}-D_{1,47} \text {, } \\
& -D_{1,6}+D_{1,34},-D_{1,7}+D_{1,45}, D_{1,13}-D_{1,26}, D_{1,15}+D_{1,27}, D_{1,16}+D_{1,23} \text {, } \\
& \left.D_{1,17}-D_{1,25}, D_{1,35}-D_{1,67}, D_{1,37}-D_{1,56}\right\} \text {, } \\
& \mathfrak{k}_{2 \xi_{1}}=\operatorname{span}_{\mathbf{R}}\left\{\frac{1}{2}\left(-D_{1,4}-D_{1,12}+D_{1,36}-D_{1,57}\right)+\sqrt{-1} R\left(e_{3}-e_{1}\right)\right\}, \\
& \mathfrak{k}_{2 \xi_{2}}=\operatorname{span}_{\mathbf{R}}\left\{\frac{1}{2}\left(D_{1,4}+D_{1,12}-D_{1,36}+D_{1,57}\right)+\sqrt{-1} R\left(e_{3}-e_{1}\right)\right\}, \\
& \mathfrak{k}_{\xi_{1}+\xi_{2}}=\operatorname{span}_{\mathbf{R}}\left\{-D_{1,1}-D_{1,24}-D_{1,37}-D_{1,56}=2 D_{2,1},\right. \\
& -D_{1,2}+D_{1,14}-D_{1,35}-D_{1,67}=2 D_{2,2} \text {, } \\
& -D_{1,3}-D_{1,46}+D_{1,17}+D_{1,25}=2 D_{2,3} \text {, } \\
& -D_{1,5}+D_{1,47}+D_{1,16}-D_{1,23}=2 D_{2,5} \text {, } \\
& -D_{1,6}-D_{1,34}-D_{1,15}+D_{1,27}=2 D_{2,6} \text {, } \\
& \left.-D_{1,7}-D_{1,45}-D_{1,13}-D_{1,26}=2 D_{2,7}\right\} \text {, }
\end{aligned}
$$




$$
\begin{aligned}
& \mathfrak{k}_{\xi_{1}-\xi_{2}}=\operatorname{span}_{\mathbf{R}}\left\{-D_{1,1}-D_{1,24}+D_{1,37}+D_{1,56}=2 D_{2,24},\right. \\
& -D_{1,2}+D_{1,14}+D_{1,35}+D_{1,67}=2 D_{2,14} \\
& -D_{1,3}-D_{1,46}-D_{1,25}-D_{1,17}=-2 D_{2,46} \text {, } \\
& -D_{1,5}+D_{1,47}-D_{1,16}+D_{1,23}=2 D_{2,47} \text {, } \\
& -D_{1,6}-D_{1,34}+D_{1,15}-D_{1,27}=-2 D_{2,34} \text {, } \\
& \left.-D_{1,7}-D_{1,45}+D_{1,13}+D_{1,26}=-2 D_{2,45}\right\} \\
& \mathfrak{k}_{\xi_{1}}=\operatorname{span}_{\mathbf{R}}\left\{D\left(x_{1} \bar{u}_{1}\right)+\sqrt{-1} R\left(y_{1} u_{1}\right) \mid\left(x_{1}, y_{1}\right)=\left(1, c_{4}\right),\left(c_{1},-c_{2}\right),\left(c_{2}, c_{1}\right),\right. \\
& \left.\left(c_{3}, c_{6}\right),\left(c_{4},-1\right),\left(c_{5},-c_{7}\right),\left(c_{6},-c_{3}\right),\left(c_{7}, c_{5}\right)\right\}, \\
& \mathfrak{k}_{\xi_{2}}=\operatorname{span}_{\mathbf{R}}\left\{D\left(x_{1} \bar{u}_{1}\right)+\sqrt{-1} R\left(y_{1} u_{1}\right) \mid\left(x_{1}, y_{1}\right)=\left(1,-c_{4}\right),\left(c_{1}, c_{2}\right),\left(c_{2},-c_{1}\right),\right. \\
& \left.\left(c_{3},-c_{6}\right),\left(c_{4}, 1\right),\left(c_{5}, c_{7}\right),\left(c_{6}, c_{3}\right),\left(c_{7},-c_{5}\right)\right\}, \\
& \mathfrak{p}_{2 \xi_{1}}=\operatorname{span}_{\mathbf{R}}\left\{D\left(c_{4} \bar{u}_{2}\right)-\sqrt{-1} R u_{2}\right\}, \\
& \mathfrak{p}_{2 \xi_{2}}=\operatorname{span}_{\mathbf{R}}\left\{D\left(c_{4} \bar{u}_{2}\right)+\sqrt{-1} R u_{2}\right\}, \\
& \mathfrak{p}_{\xi_{1}+\xi_{2}}=\operatorname{span}_{\mathbf{R}}\left\{D\left(c_{i} \bar{u}_{2}\right), i=1,2,3,5,6,7\right\}, \\
& \mathfrak{p}_{\xi_{1}-\xi_{2}}=\operatorname{span}_{\mathbf{R}}\left\{\sqrt{-1} R\left(c_{i} u_{2}\right), i=1,2,3,5,6,7\right\}, \\
& \mathfrak{p}_{\xi_{1}}=\operatorname{span}_{\mathbf{R}}\left\{D\left(x_{3} \bar{u}_{3}\right)+\sqrt{-1} R\left(y_{3} u_{3}\right) \mid\left(x_{3}, y_{3}\right)=\left(1, c_{4}\right),\left(c_{1}, c_{2}\right),\left(c_{2}, c_{1}\right),\right. \\
& \left.\left(c_{3}, c_{6}\right),\left(c_{4},-1\right),\left(c_{5},-c_{7}\right),\left(c_{6},-c_{3}\right),\left(c_{7}, c_{5}\right)\right\}, \\
& \mathfrak{p}_{\xi_{2}}=\operatorname{span}_{\mathbf{R}}\left\{D\left(x_{3} \bar{u}_{3}\right)+\sqrt{-1} R\left(y_{3} u_{3}\right) \mid\left(x_{3}, y_{3}\right)=\left(1,-c_{4}\right),\left(c_{1},-c_{2}\right),\left(c_{2}, c_{1}\right),\right. \\
& \left.\left(c_{3}, c_{6}\right),\left(c_{4}, 1\right),\left(c_{5},-c_{7}\right),\left(c_{6},-c_{3}\right),\left(c_{7}, c_{5}\right)\right\} \text {. }
\end{aligned}
$$

Thus we see that

$$
\begin{aligned}
\mathfrak{k}_{0}= & \mathfrak{k}_{0}^{\prime}+\mathfrak{c}\left(\mathfrak{k}_{0}\right)=\mathfrak{k}_{0}^{\prime}+\mathbf{R} \sqrt{-1} R\left(e_{1}-2 e_{2}+e_{3}\right) \cong \mathfrak{s o}(6)+\mathbf{R}, \\
\mathfrak{k}_{1}:= & \mathfrak{k}_{0}+\mathfrak{k}_{2 \xi_{1}}+\mathfrak{k}_{2 \xi_{2}} \\
= & \mathfrak{k}_{0}^{\prime}+\mathbf{R} \sqrt{-1} R\left(e_{1}-2 e_{2}+e_{3}\right)+\mathbf{R}\left(D_{1,4}+D_{1,12}-D_{1,36}+D_{1,57}\right) \\
& +\mathbf{R} \sqrt{-1} R\left(e_{3}-e_{1}\right) \\
\cong & \mathfrak{s o}(6)+\mathbf{R}+\mathbf{R}+\mathbf{R},
\end{aligned}
$$




$$
\begin{aligned}
\mathfrak{k}_{2} & :=\mathfrak{k}_{1}+\mathfrak{k}_{\xi_{1}+\xi_{2}}+\mathfrak{k}_{\xi_{1}-\xi_{2}}=\mathfrak{D}_{0}+\sqrt{-1} \mathfrak{R}_{0} \\
& =\mathfrak{D}_{0}+\mathbf{R} \sqrt{-1} R\left(e_{1}-2 e_{2}+e_{3}\right)+\mathbf{R} \sqrt{-1} R\left(e_{3}-e_{1}\right) \\
& =\mathfrak{D}_{0}+\mathbf{R} \sqrt{-1} R\left(e_{2}-e_{3}\right)+\mathbf{R} \sqrt{-1} R\left(2 e_{1}-e_{2}-e_{3}\right) \\
& \cong \mathfrak{s o}(8)+\mathbf{R}+\mathbf{R}, \\
\mathfrak{k} & :=\mathfrak{k}_{2}+\mathfrak{k}_{\xi_{1}}+\mathfrak{k}_{\xi_{2}}=\mathfrak{D}_{0}+\sqrt{-1} \mathfrak{R}_{0}+\mathfrak{D}_{1}+\sqrt{-1} \mathfrak{R}_{1} \\
& =\left(\mathfrak{D}_{0}+\mathfrak{D}_{1}+\sqrt{-1} \mathfrak{R}_{1}+\mathbf{R} \sqrt{-1} R\left(e_{2}-e_{3}\right)\right)+\mathbf{R} \sqrt{-1} R\left(2 e_{1}-e_{2}-e_{3}\right) \\
& =\mathfrak{k}^{\prime}+\mathfrak{c}(\mathfrak{k}) \cong \mathfrak{s o}(10)+\mathbf{R} .
\end{aligned}
$$

Consider the subgroup

$$
\tilde{K}_{2}=U(1) \times \operatorname{Spin}(2) \times \operatorname{Spin}(8) \subset \tilde{K}=U(1) \times \operatorname{Spin}(10),
$$

where $U(1)$ is given by (37), $\operatorname{Spin}(2) \subset \operatorname{Spin}(10) \cong\left(E_{6}\right)_{e_{1}}$ is generated by

$$
\begin{aligned}
\alpha_{23}(t) & :=\exp \left(t \sqrt{-1} R\left(e_{2}-e_{3}\right)\right): \\
& \left(\begin{array}{lll}
\xi_{1} & x_{3} & \bar{x}_{2} \\
\bar{x}_{3} & \xi_{2} & x_{1} \\
x_{2} & \bar{x}_{1} & \xi_{3}
\end{array}\right) \mapsto\left(\begin{array}{ccc}
\xi_{1} & e^{\frac{t \sqrt{-1}}{2}} x_{3} & e^{-\frac{t \sqrt{-1}}{2} \bar{x}_{2}} \\
e^{\frac{t \sqrt{-1}}{2}} \bar{x}_{3} & e^{t \sqrt{-1} \xi_{2}} & x_{1} \\
e^{-\frac{t \sqrt{-1}}{2}} x_{2} & \bar{x}_{1} & e^{-t \sqrt{-1}} \xi_{3}
\end{array}\right),
\end{aligned}
$$

and $\operatorname{Spin}(8)=\left(E_{6}\right)_{e_{1}, e_{2}, e_{3}}$ whose Lie algebra is just $\mathfrak{D}_{0}$. Therefore,

$$
\operatorname{Spin}(2) \cap \operatorname{Spin}(8)=\left\{\alpha_{23}(t) \mid e^{t \sqrt{-1}}=1\right\}=\left\{\alpha_{23}(0), \alpha_{23}(2 \pi)\right\} .
$$

Then the natural projection

$$
p_{2}: \operatorname{Spin}(2) \times \operatorname{Spin}(8) \ni\left(\alpha_{23}(t), \beta\right) \mapsto \alpha_{23}(t) \beta \in K_{2}^{\prime}
$$

has the kernel

$$
\begin{aligned}
\operatorname{Ker} p_{2} & =\left\{\left(\alpha_{23}(t), \alpha_{23}(t)^{-1}\right) \mid t=2 k \pi, k \in \mathbf{Z}\right\} \\
& =\left\{\left(\alpha_{23}(0), \alpha_{23}(0)\right),\left(\alpha_{23}(2 \pi), \alpha_{23}(2 \pi)\right)\right\} \cong \mathbf{Z}_{2} .
\end{aligned}
$$

Hence $K_{2}^{\prime} \cong(\operatorname{Spin}(2) \times \operatorname{Spin}(8)) / \mathbf{Z}_{2}$.

On the other hand, we also have

$$
\tilde{K}_{2}=S^{1} \times \operatorname{Spin}(2) \times \operatorname{Spin}(8),
$$

where this $S^{1}$ is generated by

$$
\begin{aligned}
& \exp \left(t \sqrt{-1} R\left(e_{1}-2 e_{2}+e_{3}\right)\right): \\
& \left(\begin{array}{lll}
\xi_{1} & x_{3} & \bar{x}_{2} \\
\bar{x}_{3} & \xi_{2} & x_{1} \\
x_{2} & \bar{x}_{1} & \xi_{3}
\end{array}\right) \mapsto\left(\begin{array}{lll}
e^{t \sqrt{-1}} \xi_{1} & e^{-\frac{t \sqrt{-1}}{2}} x_{3} & e^{t \sqrt{-1}} \bar{x}_{2} \\
e^{-\frac{t \sqrt{-1}}{2}} \bar{x}_{3} & e^{-2 t \sqrt{-1}} \xi_{2} & e^{-\frac{t \sqrt{-1}}{2}} x_{1} \\
e^{t \sqrt{-1}} x_{2} & e^{-\frac{t \sqrt{-1}}{2} \bar{x}_{1}} & e^{t \sqrt{-1}} \xi_{3}
\end{array}\right)
\end{aligned}
$$


$\operatorname{Spin}(2) \subset E_{6}$ is generated by

$$
\begin{aligned}
\alpha_{31}(t) & :=\exp \left(t \sqrt{-1} R\left(e_{3}-e_{1}\right)\right): \\
& \left(\begin{array}{lll}
\xi_{1} & x_{3} & \bar{x}_{2} \\
\bar{x}_{3} & \xi_{2} & x_{1} \\
x_{2} & \bar{x}_{1} & \xi_{3}
\end{array}\right) \mapsto\left(\begin{array}{ccc}
e^{-t \sqrt{-1}} \xi_{1} & e^{-\frac{t \sqrt{-1}}{2}} x_{3} & \bar{x}_{2} \\
e^{-\frac{t \sqrt{-1}}{2} \bar{x}_{3}} & \xi_{2} & e^{\frac{t \sqrt{-1}}{2}} x_{1} \\
x_{2} & e^{\frac{t \sqrt{-1}}{2}} \bar{x}_{1} & e^{t \sqrt{-1}} \xi_{3}
\end{array}\right),
\end{aligned}
$$

and $\operatorname{Spin}(8)=\left(E_{6}\right)_{e_{1}, e_{2}, e_{3}}$. Here $\operatorname{Spin}(2) \times \operatorname{Spin}(8) \subset\left(E_{6}\right)_{e_{2}} \cong \operatorname{Spin}(10)$. Similarly, here

$$
\operatorname{Spin}(2) \cap \operatorname{Spin}(8)=\left\{\alpha_{31}(t) \mid e^{t \sqrt{-1}}=1\right\}=\left\{\alpha_{31}(0), \alpha_{31}(2 \pi)\right\} .
$$

Then the natural projection

$$
p_{2}^{\prime}: \operatorname{Spin}(2) \times \operatorname{Spin}(8) \ni\left(\alpha_{31}(t), \beta\right) \mapsto \alpha_{31}(t) \beta \in K_{2}^{\prime}
$$

has the kernel

$$
\begin{aligned}
\operatorname{Ker} p_{2}^{\prime} & =\left\{\left(\alpha_{31}(t), \alpha_{31}(t)^{-1}\right) \mid t=2 k \pi, k \in \mathbf{Z}\right\} \\
& =\left\{\left(\alpha_{31}(0), \alpha_{31}(0)\right),\left(\alpha_{31}(2 \pi), \alpha_{31}(2 \pi)\right)\right\} \cong \mathbf{Z}_{2} .
\end{aligned}
$$

Thus,

$$
\begin{aligned}
K_{2} & =\left(S^{1} \times(\operatorname{Spin}(2) \cdot \operatorname{Spin}(8))\right) / \mathbf{Z}_{4}, \\
\operatorname{Spin}(2) \cdot \operatorname{Spin}(8) & =(\operatorname{Spin}(2) \times \operatorname{Spin}(8)) / \mathbf{Z}_{2} .
\end{aligned}
$$

Furthermore, we have

$$
\operatorname{Spin}(8) \supset \operatorname{Spin}(2) \cdot \operatorname{Spin}(6) \cong(\operatorname{Spin}(2) \times \operatorname{Spin}(6)) / \mathbf{Z}_{2},
$$

where

$$
\begin{aligned}
\operatorname{Spin}(8)=\left\{\left(\alpha_{1}, \alpha_{2}, \alpha_{3}\right)\right. & \in S O(\mathbf{K}) \times S O(\mathbf{K}) \times S O(\mathbf{K}) \mid \\
& \left.\left(\alpha_{1} x\right)\left(\alpha_{2} y\right)=\overline{\alpha_{3}(\overline{x y})} \text { for each } x, y \in \mathbf{K}\right\}
\end{aligned}
$$

acts on $H_{3}(\mathbf{K})$ by

$$
\left(\alpha_{1}, \alpha_{2}, \alpha_{3}\right)\left(\begin{array}{ccc}
\xi_{1} & x_{3} & \bar{x}_{2} \\
\bar{x}_{3} & \xi_{2} & x_{1} \\
x_{2} & \bar{x}_{1} & \xi_{3}
\end{array}\right):=\left(\begin{array}{ccc}
\xi_{1} & \alpha_{3} x_{3} & \overline{\alpha_{2} x_{2}} \\
\overline{\alpha_{3} x_{3}} & \xi_{2} & \alpha_{1} x_{1} \\
\alpha_{2} x_{2} & \overline{\alpha_{1} x_{1}} & \xi_{3}
\end{array}\right),
$$

$$
\operatorname{Spin}(2):=\left\{\left(\alpha_{1}, \alpha_{2}, \alpha_{3}\right) \in \operatorname{Spin}(8) \mid \alpha_{2}\left(c_{i}\right)=c_{i} \text {, if } i \neq 0,4\right\}
$$

is generated by $D_{1,4}+D_{1,12}-D_{1,36}+D_{1,57}$ and

$$
\operatorname{Spin}(6):=\left\{\left(\alpha_{1}, \alpha_{2}, \alpha_{3}\right) \in \operatorname{Spin}(8) \mid \alpha_{2}(1)=1, \alpha_{2}\left(c_{4}\right)=c_{4}\right\}
$$

is generated by $\mathfrak{k}_{0}^{\prime}$. Note that

$$
\operatorname{Spin}(2) \cap \operatorname{Spin}(6)=\{(\mathrm{Id}, \mathrm{Id}, \mathrm{Id}),(-\mathrm{Id}, \mathrm{Id},-\mathrm{Id})\},
$$


we see that $\mathbf{Z}_{2}=\{((\mathrm{Id}, \mathrm{Id}, \mathrm{Id}),(\mathrm{Id}, \mathrm{Id}, \mathrm{Id})),((-\mathrm{Id}, \mathrm{Id},-\mathrm{Id}),(-\mathrm{Id}, \mathrm{Id},-\mathrm{Id}))\}$. Thus, a connected compact Lie subgroup $K_{1}$ of $K$ generated by $\mathfrak{k}_{1}$ is

$$
K_{1}=\left(S^{1} \times(\operatorname{Spin}(2) \cdot(\operatorname{Spin}(2) \cdot \operatorname{Spin}(6)))\right) / \mathbf{Z}_{4} .
$$

Moreover,

$$
S^{1} \cap \operatorname{Spin}(6)=\{(\mathrm{Id}, \mathrm{Id}, \mathrm{Id}),(-\mathrm{Id}, \mathrm{Id},-\mathrm{Id})\},
$$

hence a connected compact Lie group $K_{0}$ of $K$ generated by $\mathfrak{k}_{0}$ is

$$
K_{0}=\left(S^{1} \times \operatorname{Spin}(6)\right) / \mathbf{Z}_{2},
$$

where $\mathbf{Z}_{2}=\{((\mathrm{Id}, \mathrm{Id}, \mathrm{Id}),(\mathrm{Id}, \mathrm{Id}, \mathrm{Id})),((-\mathrm{Id}, \mathrm{Id},-\mathrm{Id}),(-\mathrm{Id}, \mathrm{Id},-\mathrm{Id}))\}$.

5.7. Isotropy representation of $\left(E_{6}, U(1) \cdot \operatorname{Spin}(10)\right)$. Via the linear isomorphism $p_{*}: \mathfrak{p} \rightarrow T_{o}$ (EIII) given by (39), we can describe the isotropy representation of $\left(E_{6}, U(1)\right.$. $\operatorname{Spin}(10))$.

Lemma 5.2. (1) For each $a \in K$ and each $\xi \in \mathfrak{p}$,

$$
p_{*}(\operatorname{Ad}(a) \xi)=(\operatorname{Ad}(a) \xi)\left(e_{1}\right)=\left(a \circ \xi \circ a^{-1}\right)\left(e_{1}\right) .
$$

(2) For each $T \in \mathfrak{k}$ and each $\xi \in \mathfrak{p}$,

$$
p_{*}(\operatorname{ad}(T) \xi)=p_{*}([T, \xi])=([T, \xi])\left(e_{1}\right) .
$$

The restriction $\left(\rho_{K}, V=H_{3}\left(\mathbf{K}^{\mathbf{C}}\right)\right)$ of Chevally-Schafer's representation $\left(\tilde{\rho}, H_{3}\left(\mathbf{K}^{\mathbf{C}}\right)\right)$ of $E_{6}$ to $K$ can be decomposed into three irreducible representations

$$
\left(\rho_{K}, V\right)=\left(\rho_{1}, V_{1}\right) \oplus\left(\rho_{2}, V_{2}\right) \oplus\left(\rho_{3}, V_{3}\right),
$$

where $V_{1}, V_{2}$ and $V_{3}$ are given as follows:

$$
\begin{aligned}
& V_{1}=\left\{\xi e_{1} \mid \xi \in \mathbf{C}\right\}, \\
& V_{2}=\left(H_{3}\left(\mathbf{K}^{\mathbf{C}}\right)\right)_{-\sigma}=\left\{x_{2} u_{2}+x_{3} u_{3} \mid x_{2}, x_{3} \in \mathbf{K}^{\mathbf{C}}\right\} \cong T_{o}(\mathrm{EIII}), \\
& V_{3}=H_{2}\left(\mathbf{K}^{\mathbf{C}}\right)=\left\{\xi_{2} e_{2}+\xi_{3} e_{3}+x_{1} u_{1} \mid x_{1} \in \mathbf{K}^{\mathbf{C}}, \xi_{2}, \xi_{3} \in \mathbf{C}\right\},
\end{aligned}
$$

and $V_{1} \oplus V_{3}=\left(H_{3}\left(\mathbf{K}^{\mathbf{C}}\right)\right)_{\sigma}$. Note that $\rho_{1}$ is a scalar representation, the restriction of $\rho_{2}$ to $\operatorname{Spin}(10)$ is equivalent to one of the half-spin representations of $\operatorname{Spin}(10, \mathbf{C})$, denoted by $\triangle_{10}^{+}$, and the restriction of $\rho_{3}$ to $\operatorname{Spin}(10)$ is equivalent to the standard representation of $\operatorname{Spin}(10, \mathbf{C})$.

Now we discuss the linear isotropy action of an element $\phi(\theta)=\exp \left(t \sqrt{-1} R\left(2 e_{1}-\right.\right.$ $\left.\left.e_{2}-e_{3}\right)\right): H_{3}\left(\mathbf{K}^{\mathbf{C}}\right) \rightarrow H_{3}\left(\mathbf{K}^{\mathbf{C}}\right)$ generating the center $U(1)$ of $K$ on both $\mathfrak{p}$ and $V_{2}=$ $\left(H_{3}\left(\mathbf{K}^{\mathbf{C}}\right)\right)_{-\sigma}$, which are linearly isomorphic to $T_{o}(\mathrm{EIII})$. Using the formula (38) and Lemma 
5.2, we compute

$$
\begin{aligned}
& p_{*}\left(\operatorname{Ad}(\phi(\theta)) D\left(x_{2} \bar{u}_{2}\right)\right)=\theta^{-3} p_{*}\left(D\left(x_{2} \bar{u}_{2}\right)\right), \\
& p_{*}\left(\operatorname{Ad}(\phi(\theta)) R\left(x_{2} u_{2}\right)\right)=\theta^{-3} p_{*}\left(R\left(x_{2} u_{2}\right)\right), \\
& p_{*}\left(A d(\phi(\theta)) D\left(x_{3} \bar{u}_{3}\right)\right)=\theta^{-3} p_{*}\left(D\left(x_{3} \bar{u}_{3}\right)\right), \\
& p_{*}\left(A d(\phi(\theta)) R\left(x_{3} u_{3}\right)\right)=\theta^{-3} p_{*}\left(R\left(x_{3} u_{3}\right)\right) .
\end{aligned}
$$

On the other hand, the tangent vector space $T_{o}$ (EIII) at $o=\left[e_{1}\right] \in$ EIII $\subset P\left(H_{3}(\mathbf{K})^{\mathbf{C}}\right)$ is linearly isomorphic to a vector subspace $V_{2}=\left(H_{3}\left(\mathbf{K}^{\mathbf{C}}\right)\right)_{-\sigma}$, which is a horizontal vector subspace at a point $e_{1}$ under the Hopf fibration $H_{3}(\mathbf{K})^{\mathbf{C}} \supset S^{53}(1) \rightarrow P\left(H_{3}(\mathbf{K}){ }^{\mathbf{C}}\right)$. By the formula (38) we see that a vector $x_{2} u_{2}+x_{3} u_{3} \in\left(H_{3}\left(\mathbf{K}^{\mathbf{C}}\right)\right)_{-\sigma}$ at a point $e_{1}$ in a vector space $H_{3}(\mathbf{K})^{\mathbf{C}}$ representing a tangent vector of EIII at $o=\left[e_{1}\right]$ is moved by the linear action of $\phi(\theta)$ to a vector $\theta x_{2} u_{2}+\theta x_{3} u_{3} \in\left(H_{3}\left(\mathbf{K}^{\mathbf{C}}\right)\right)_{-\sigma}$ at $\theta^{4} e_{1}$. Thus its corresponding tangent vector of EIII at $o=\left[e_{1}\right]$ must be $\theta^{-4}\left(\theta x_{2} u_{2}+\theta x_{3} u_{3}\right)=\theta^{-3}\left(x_{2} u_{2}+x_{3} u_{3}\right) \in V_{2}=$ $\left(H_{3}\left(\mathbf{K}^{\mathbf{C}}\right)\right)_{-\sigma}$ at $e_{1}$. Hence the linear isotropy action of $\phi(\theta)$ on $V_{2}=\left(H_{3}\left(\mathbf{K}^{\mathbf{C}}\right)\right)_{-\sigma}$ is given by the multiplication by $\theta^{-3}$ on $V_{2}=\left(H_{3}\left(\mathbf{K}^{\mathbf{C}}\right)\right)_{-\sigma}$. Therefore the linear isotropy representation of $\left(E_{6}, U(1) \cdot \operatorname{Spin}(10)\right)$ is $\left(\mu_{3} \otimes_{\mathbf{C}} \triangle_{10}^{+}\right)_{\mathbf{R}}$.

5.8. The subgroup $K_{[\mathfrak{a}]}$. The maximal abelian subspace $\mathfrak{a}$ of $\mathfrak{p}$ is described as follows:

$$
\mathfrak{a}=\mathbf{R} H_{1} \oplus \mathbf{R} H_{2}=\mathbf{R}\left(D \bar{u}_{2}+\sqrt{-1} R\left(\mathbf{c}_{4} u_{2}\right)\right) \oplus \mathbf{R}\left(D \bar{u}_{2}-\sqrt{-1} R\left(\mathbf{c}_{4} u_{2}\right)\right)
$$

and

$$
p_{*}(\mathfrak{a})=\mathbf{R}\left(1+\sqrt{-1} \mathbf{c}_{4}\right) u_{2} \oplus \mathbf{R}\left(1-\sqrt{-1} \mathbf{c}_{4}\right) u_{2} .
$$

We shall use the map $\varphi: S p(4) \rightarrow E_{6}$ given by Yokota ([27]) and known results for the case $(\check{U}, \check{K})=(S p(4), S p(2) \times S p(2))$ in order to describe a generator of $K_{[\mathfrak{a}]}$.

The Cayley algebra $\mathbf{K}$ naturally contains the field $\mathbf{H}$ of quaternions as

$$
\mathbf{H}=\left\{x_{0}+x_{2} c_{2}+x_{3} c_{3}+x_{5} c_{5} \mid x_{i} \in \mathbf{R}\right\} .
$$

Any element $x \in \mathbf{K}$ can be expressed by

$$
\begin{aligned}
x & =x_{0}+x_{1} c_{1}+x_{2} c_{2}+x_{3} c_{3}+x_{4} c_{4}+x_{5} c_{5}+x_{6} c_{6}+x_{7} c_{7} \\
& =\left(x_{0}+x_{2} c_{2}+x_{3} c_{3}+x_{5} c_{5}\right)+\left(x_{4}+x_{1} c_{2}+x_{6} c_{3}-x_{7} c_{5}\right) c_{4} \\
& =: m+a \mathbf{e} \in \mathbf{H} \oplus \mathbf{H e}=\mathbf{K},
\end{aligned}
$$

where we set $m:=x_{0}+x_{2} c_{2}+x_{3} c_{3}+x_{5} c_{5} \in \mathbf{H}, a:=x_{4}+x_{1} c_{2}+x_{6} c_{3}-x_{7} c_{5} \in \mathbf{H}$ and $\mathbf{e}:=c_{4}$. In $\mathbf{H} \oplus \mathbf{H e}$, we define a multiplication by

$$
(m+a \mathbf{e})(n+b \mathbf{e})=(m n-\bar{b} a)+(a \bar{n}+b m) \mathbf{e} .
$$

More explicitly,

$$
(a \mathbf{e}) n=(a \bar{n}) \mathbf{e}, \quad m(b \mathbf{e})=(b m) \mathbf{e}, \quad(a \mathbf{e})(b \mathbf{e})=-\bar{b} a .
$$


We can also define a conjugation and an $\mathbf{R}$-linear transformation $\gamma$ on $\mathbf{H} \oplus \mathbf{H e}$ respectively by

$$
\overline{m+a \mathbf{e}}=\bar{m}-a \mathbf{e}, \quad \gamma(m+a \mathbf{e})=m-a \mathbf{e} .
$$

Thus $\gamma \in G_{2} \subset F_{4}$. Any element

$$
X=\left(\begin{array}{lll}
\xi_{1} & x_{3} & \bar{x}_{2} \\
\bar{x}_{3} & \xi_{2} & x_{1} \\
x_{2} & \bar{x}_{1} & \xi_{3}
\end{array}\right)=\left(\begin{array}{ccc}
\xi_{1} & m_{3} & \bar{m}_{2} \\
\bar{m}_{3} & \xi_{2} & m_{1} \\
m_{2} & \bar{m}_{1} & \xi_{3}
\end{array}\right)+\left(\begin{array}{ccc}
0 & a_{3} \mathbf{e} & -a_{2} \mathbf{e} \\
-a_{3} \mathbf{e} & 0 & a_{1} \mathbf{e} \\
a_{2} \mathbf{e} & -a_{1} \mathbf{e} & 0
\end{array}\right),
$$

of $H_{3}(\mathbf{K})$, where $x_{i}=m_{i}+a_{i} \mathbf{e} \in \mathbf{H} \oplus \mathbf{H e}=\mathbf{K}$ and $\xi_{i} \in \mathbf{R}$, can be identified with an element

$$
\left(\begin{array}{ccc}
\xi_{1} & m_{3} & \bar{m}_{2} \\
\bar{m}_{3} & \xi_{2} & m_{1} \\
m_{2} & \bar{m}_{1} & \xi_{3}
\end{array}\right)+\left(a_{1}, a_{2}, a_{3}\right)
$$

in $H_{3}(\mathbf{H}) \oplus \mathbf{H}^{3}$. Hereafter, we often use an identification $H_{3}(\mathbf{K}) \cong H_{3}(\mathbf{H}) \oplus \mathbf{H}^{3}$.

Let the $\mathbf{C}$-linear mapping $\gamma: H_{3}\left(\mathbf{K}^{\mathbf{C}}\right) \rightarrow H_{3}\left(\mathbf{K}^{\mathbf{C}}\right)$ be the complexification of $\gamma \in$ $G_{2} \subset F_{4}$. Then $\gamma \in E_{6}$ and $\gamma^{2}=1$. Recall that $\tau$ is the complex conjugation of $H_{3}\left(\mathbf{K}^{\mathbf{C}}\right)$ with respect to $H_{3}(\mathbf{K})$. Consider an involutive complex conjugate linear transformation $\tau \gamma$ of $H_{3}\left(\mathbf{K}^{\mathbf{C}}\right)$ and the following subgroup $\left(E_{6}\right)^{\tau \gamma}$ of $E_{6}$ :

$$
\left(E_{6}\right)^{\tau \gamma}=\left\{\alpha \in E_{6} \mid \tau \gamma \alpha=\alpha \tau \gamma\right\} .
$$

Correspondingly, $H_{3}\left(\mathbf{K}^{\mathbf{C}}\right)$ can be decomposed into the following two real vector subspaces:

$$
H_{3}\left(\mathbf{K}^{\mathbf{C}}\right)=\left(H_{3}\left(\mathbf{K}^{\mathbf{C}}\right)\right)_{\tau \gamma} \oplus\left(H_{3}\left(\mathbf{K}^{\mathbf{C}}\right)\right)_{-\tau \gamma},
$$

where

$$
\begin{aligned}
& \left(H_{3}\left(\mathbf{K}^{\mathbf{C}}\right)\right)_{\tau \gamma}:=\left\{X \in H_{3}\left(\mathbf{K}^{\mathbf{C}}\right) \mid \tau \gamma X=X\right\} \\
& =\left\{\left(\begin{array}{lll}
\xi_{1} & m_{3} & \bar{m}_{2} \\
\bar{m}_{3} & \xi_{2} & m_{1} \\
m_{2} & \bar{m}_{1} & \xi_{3}
\end{array}\right)+\sqrt{-1}\left(\begin{array}{ccc}
0 & a_{3} e & -a_{2} e \\
-a_{3} e & 0 & a_{1} e \\
a_{2} e & -a_{1} e & 0
\end{array}\right) \mid \xi_{i} \in \mathbf{R}, m_{i}, a_{i} \in \mathbf{H}\right\} \\
& =H_{3}(\mathbf{H}) \oplus \sqrt{-1} \mathbf{H}^{3}, \\
& \left(H_{3}\left(\mathbf{K}^{\mathbf{C}}\right)\right)_{-\tau \gamma}:=\left\{X \in H_{3}\left(\mathbf{K}^{\mathbf{C}}\right) \mid \tau \gamma X=-X\right\} \\
& =\left\{\sqrt{-1}\left(\begin{array}{ccc}
\xi_{1} & m_{3} & \bar{m}_{2} \\
\bar{m}_{3} & \xi_{2} & m_{1} \\
m_{2} & \bar{m}_{1} & \xi_{3}
\end{array}\right)+\left(\begin{array}{ccc}
0 & a_{3} e & -a_{2} e \\
-a_{3} e & 0 & a_{1} e \\
a_{2} e & -a_{1} e & 0
\end{array}\right) \mid \xi_{i} \in \mathbf{R}, m_{i}, a_{i} \in \mathbf{H}\right\} \\
& =\sqrt{-1} H_{3}(\mathbf{H}) \oplus \mathbf{H}^{3} .
\end{aligned}
$$

In particular, $H_{3}\left(\mathbf{K}^{\mathbf{C}}\right)=\left(\left(H_{3}\left(\mathbf{K}^{\mathbf{C}}\right)\right)_{\tau \gamma}\right)$.

Let $H_{4}(\mathbf{H})_{0}:=\left\{P \in H_{4}(\mathbf{H}) \mid \operatorname{tr} P=0\right\}$. Define a $\mathbf{C}$-linear isomorphism $g: H_{3}\left(\mathbf{K}^{\mathbf{C}}\right)=$ $H_{3}\left(\mathbf{H}^{\mathbf{C}}\right) \oplus\left(\mathbf{H}^{3}\right)^{\mathbf{C}} \rightarrow H_{4}(\mathbf{H})_{0}^{\mathbf{C}}$ by

$$
g(M+\mathbf{a}):=\left(\begin{array}{cc}
\frac{1}{2} \operatorname{tr}(M) & \sqrt{-1} \mathbf{a} \\
\sqrt{-1} \mathbf{a}^{*} & M-\frac{1}{2} \operatorname{tr}(M) \mathrm{I}
\end{array}\right)
$$


for $M+\mathbf{a} \in H_{3}\left(\mathbf{K}^{\mathbf{C}}\right)$. Then we have

$$
g\left(\left(H_{3}\left(\mathbf{K}^{\mathbf{C}}\right)\right)_{\tau \gamma}\right)=H_{4}(\mathbf{H})_{0}, \quad g\left(\left(H_{3}\left(\mathbf{K}^{\mathbf{C}}\right)\right)_{-\tau \gamma}\right)=\sqrt{-1} H_{4}(\mathbf{H})_{0} .
$$

The map $\varphi: S p(4) \longrightarrow\left(E_{6}\right)^{\tau \gamma} \subset E_{6}$, defined by $\varphi(A) X:=g^{-1}\left(A(g X) A^{*}\right)$ for each $X \in H_{3}\left(\mathbf{K}^{\mathbf{C}}\right)$, is a surjective Lie group homomorphism and $\operatorname{Ker}(\varphi)=\{\mathrm{I},-\mathrm{I}\} \cong \mathbf{Z}_{2}$. Therefore we obtain

$$
\operatorname{Sp}(4) / \mathbf{Z}_{2} \cong\left(E_{6}\right)^{\tau \gamma}
$$

Consider real vector subspaces $\left(H_{3}\left(\mathbf{K}^{\mathbf{C}}\right)\right)_{\tau \gamma, \sigma},\left(H_{3}\left(\mathbf{K}^{\mathbf{C}}\right)\right)_{\tau \gamma,-\sigma}$ of $\left(H_{3}\left(\mathbf{K}^{\mathbf{C}}\right)\right)_{\tau \gamma}$ and $\left(H_{3}\left(\mathbf{K}^{\mathbf{C}}\right)\right)_{-\tau \gamma, \sigma}$, $\left(H_{3}\left(\mathbf{K}^{\mathbf{C}}\right)\right)_{-\tau \gamma,-\sigma}$ of $\left(H_{3}\left(\mathbf{K}^{\mathbf{C}}\right)\right)_{-\tau \gamma}$, which are eigenspaces of $\sigma$, respectively given by

$$
\begin{aligned}
& \left(H_{3}\left(\mathbf{K}^{\mathbf{C}}\right)\right)_{\tau \gamma, \sigma}=\left\{X \in H_{3}\left(\mathbf{K}^{\mathbf{C}}\right) \mid \tau \gamma X=X, \sigma X=X\right\} \\
& =\left\{\left(\begin{array}{ccc}
\xi_{1} & 0 & 0 \\
0 & \xi_{2} & m_{1} \\
0 & \bar{m}_{1} & \xi_{3}
\end{array}\right)+\sqrt{-1}\left(\begin{array}{ccc}
0 & 0 & 0 \\
0 & 0 & a_{1} \mathbf{e} \\
0 & -a_{1} \mathbf{e} & 0
\end{array}\right) \mid \xi_{i} \in \mathbf{R}, m_{1}, a_{1} \in \mathbf{H}\right\}, \\
& \left(H_{3}\left(\mathbf{K}^{\mathbf{C}}\right)\right)_{\tau \gamma,-\sigma}=\left\{X \in H_{3}\left(\mathbf{K}^{\mathbf{C}}\right) \mid \tau \gamma X=X, \sigma X=-X\right\} \\
& =\left\{\left(\begin{array}{ccc}
0 & m_{3} & \bar{m}_{2} \\
\bar{m}_{3} & 0 & 0 \\
m_{2} & 0 & 0
\end{array}\right)+\sqrt{-1}\left(\begin{array}{ccc}
0 & a_{3} \mathbf{e} & -a_{2} \mathbf{e} \\
-a_{3} \mathbf{e} & 0 & 0 \\
a_{2} \mathbf{e} & 0 & 0
\end{array}\right) \mid m_{2}, m_{3}, a_{2}, a_{3} \in \mathbf{H}\right\}, \\
& \left(H_{3}\left(\mathbf{K}^{\mathbf{C}}\right)\right)_{-\tau \gamma, \sigma}=\left\{X \in H_{3}\left(\mathbf{K}^{\mathbf{C}}\right) \mid \tau \gamma X=-X, \sigma X=X\right\} \\
& =\left\{\sqrt{-1}\left(\begin{array}{ccc}
\xi_{1} & 0 & 0 \\
0 & \xi_{2} & m_{1} \\
0 & \bar{m}_{1} & \xi_{3}
\end{array}\right)+\left(\begin{array}{ccc}
0 & 0 & 0 \\
0 & 0 & a_{1} \mathbf{e} \\
0 & -a_{1} \mathbf{e} & 0
\end{array}\right) \mid \xi_{i} \in \mathbf{R}, m_{1}, a_{1} \in \mathbf{H}\right\}, \\
& \left(H_{3}\left(\mathbf{K}^{\mathbf{C}}\right)\right)_{-\tau \gamma,-\sigma}=\left\{X \in H_{3}\left(\mathbf{K}^{\mathbf{C}}\right) \mid \tau \gamma X=-X, \sigma X=-X\right\} \\
& =\left\{\sqrt{-1}\left(\begin{array}{ccc}
0 & m_{3} & \bar{m}_{2} \\
\bar{m}_{3} & 0 & 0 \\
m_{2} & 0 & 0
\end{array}\right)+\left(\begin{array}{ccc}
0 & a_{3} \mathbf{e} & -a_{2} \mathbf{e} \\
-a_{3} \mathbf{e} & 0 & 0 \\
a_{2} \mathbf{e} & 0 & 0
\end{array}\right) \mid m_{2}, m_{3}, a_{2}, a_{3} \in \mathbf{H}\right\} .
\end{aligned}
$$

Thus we have the following decompositions

$$
\begin{aligned}
\left(H_{3}\left(\mathbf{K}^{\mathbf{C}}\right)\right)_{\sigma} & =\left(H_{3}\left(\mathbf{K}^{\mathbf{C}}\right)\right)_{\tau \gamma, \sigma} \oplus\left(H_{3}\left(\mathbf{K}^{\mathbf{C}}\right)\right)_{-\tau \gamma, \sigma}, \\
\left(H_{3}\left(\mathbf{K}^{\mathbf{C}}\right)\right)_{-\sigma} & =\left(H_{3}\left(\mathbf{K}^{\mathbf{C}}\right)\right)_{\tau \gamma,-\sigma} \oplus\left(H_{3}\left(\mathbf{K}^{\mathbf{C}}\right)\right)_{-\tau \gamma,-\sigma} .
\end{aligned}
$$


Note that the images of $\left(H_{3}\left(\mathbf{K}^{\mathbf{C}}\right)\right)_{\tau \gamma, \sigma}$ and $\left(H_{3}\left(\mathbf{K}^{\mathbf{C}}\right)\right)_{\tau \gamma,-\sigma}$ of the homomorphism $g$ defined above is expressed explicitly as follows:

$$
\begin{aligned}
g\left(\left(H_{3}\left(\mathbf{K}^{\mathbf{C}}\right)\right)_{\tau \gamma, \sigma}\right) & \\
\quad= & \left\{\left(\begin{array}{cccc}
\frac{1}{2}\left(\xi_{1}+\xi_{2}+\xi_{3}\right) & -a_{1} & 0 & 0 \\
-\bar{a}_{1} & \frac{1}{2}\left(\xi_{1}-\xi_{2}-\xi_{3}\right) & 0 & 0 \\
0 & 0 & \frac{1}{2}\left(-\xi_{1}+\xi_{2}-\xi_{3}\right) & m_{1} \\
0 & 0 & \bar{m}_{1} & \frac{1}{2}\left(-\xi_{1}-\xi_{2}+\xi_{3}\right)
\end{array}\right)\right.
\end{aligned}
$$

$\left.\mid \xi_{1}, \xi_{2}, \xi_{3} \in \mathbf{R}, a_{1}, m_{1} \in \mathbf{H}\right\}$,

$g\left(\left(H_{3}\left(\mathbf{K}^{\mathbf{C}}\right)\right)_{\tau \gamma,-\sigma}\right)=\left\{\left(\begin{array}{cccc}0 & 0 & -a_{2} & -a_{3} \\ 0 & 0 & m_{3} & \bar{m}_{2} \\ -\bar{a}_{2} & \bar{m}_{3} & 0 & 0 \\ -\bar{a}_{3} & m_{2} & 0 & 0\end{array}\right) \mid a_{2}, a_{3}, m_{2}, m_{3} \in \mathbf{H}\right\}$.

For each element $A \in S p(2) \times S p(2) \subset S p(4)$, we can check that $\varphi(A) \sigma=\sigma \varphi(A)$, hence $\varphi(A) \in\left(E_{6}\right)^{\sigma}$ and we have

$$
\varphi: \operatorname{Sp}(2) \times \operatorname{Sp}(2) \longrightarrow\left(E_{6}\right)^{\tau \gamma, \sigma} \subset\left(E_{6}\right)^{\sigma} \cong U(1) \cdot \operatorname{Spin}(10) .
$$

Next, the restriction of $\varphi$ to the subgroup $S p(1) \times S p(1) \times S p(1) \times S p(1)$ gives

$$
\varphi: \operatorname{Sp}(1) \times \operatorname{Sp}(1) \times \operatorname{Sp}(1) \times \operatorname{Sp}(1) \longrightarrow\left\{\alpha \in E_{6} \mid \alpha\left(e_{i}\right)=e_{i}(i=1,2,3)\right\} \cong \operatorname{Spin}(8) .
$$

And the group $S p(1) \times S p(1)$ can be considered as the diagonal subgroup of $S p(1) \times S p(1) \times$ $S p(1) \times S p(1)$, namely, each $(a, b) \in S p(1) \times S p(1)$ corresponds to $(a, b, a, b) \in S p(1) \times$ $S p(1) \times S p(1) \times S p(1)$. Thus the restriction of $\varphi$ to $S p(1) \times S p(1)$ is mapped to a subgroup $K_{0}=S^{1} \cdot \operatorname{Spin}(6)$ of $K=E^{\sigma}=U(1) \cdot \operatorname{Spin}(10)$. In fact, for a 2-dimensional real vector subspace

$$
\tilde{\mathfrak{a}}:=\left\{\left(\begin{array}{cccc}
0 & 0 & a_{2} & 0 \\
0 & 0 & 0 & m_{2} \\
a_{2} & 0 & 0 & 0 \\
0 & m_{2} & 0 & 0
\end{array}\right) \mid a_{2}, m_{2} \in \mathbf{R}\right\} \subset g\left(\left(H_{3}\left(\mathbf{K}^{\mathbf{C}}\right)\right)_{\tau \gamma,-\sigma}\right),
$$

it follows from

$$
\begin{aligned}
& \left(\begin{array}{cccc}
a & 0 & 0 & 0 \\
0 & b & 0 & 0 \\
0 & 0 & a & 0 \\
0 & 0 & 0 & b
\end{array}\right)\left(\begin{array}{cccc}
0 & 0 & a_{2} & 0 \\
0 & 0 & 0 & m_{2} \\
a_{2} & 0 & 0 & 0 \\
0 & m_{2} & 0 & 0
\end{array}\right)\left(\begin{array}{cccc}
a^{*} & 0 & 0 & 0 \\
0 & b^{*} & 0 & 0 \\
0 & 0 & a^{*} & 0 \\
0 & 0 & 0 & b^{*}
\end{array}\right) \\
= & \left(\begin{array}{cccc}
0 & 0 & a_{2} & 0 \\
0 & 0 & 0 & m_{2} \\
a_{2} & 0 & 0 & 0 \\
0 & m_{2} & 0 & 0
\end{array}\right)
\end{aligned}
$$


that $\tilde{\mathfrak{a}}$ corresponds to the subspace

$$
\left\{\left(\begin{array}{ccc}
0 & 0 & m_{2}-\sqrt{-1} a_{2} \mathbf{e} \\
0 & 0 & 0 \\
m_{2}+\sqrt{-1} a_{2} \mathbf{e} & 0 & 0
\end{array}\right) \mid m_{2}, a_{2} \in \mathbf{R}\right\} \subset\left(H_{3}(\mathbf{K})^{\mathbf{C}}\right)_{\tau \gamma,-\sigma},
$$

which corresponds to the image $p_{*}(\mathfrak{a})$ of the maximal abelian subspace $\mathfrak{a}$ of $\mathfrak{p}$ under the linear isomorphism $p_{*}$ given by (41). It implies that $\varphi$ maps the subgroup $\check{K}_{0}=S p(1) \times S p(1)$ for the exceptional symmetric space $\left(E_{6}, S p(4) / \mathbf{Z}_{2}\right)$ of type EI to the subgroup $K_{0}=S^{1} \cdot \operatorname{Spin}(6)$ for the exceptional symmetric space $\left(E_{6}, U(1) \cdot \operatorname{Spin}(10)\right)$ of type EIII.

Recall that

$$
\check{k}:=\left(\begin{array}{cccc}
0 & 1 & 0 & 0 \\
1 & 0 & 0 & 0 \\
0 & 0 & 0 & -1 \\
0 & 0 & 1 & 0
\end{array}\right) \in \check{K}_{[\check{a}]}=(S p(1) \times S p(1)) \cdot \mathbf{Z}_{4}
$$

is a generator of $\mathbf{Z}_{4}$. Its adjoint actions on $g\left(\left(H_{3}\left(\mathbf{K}^{\mathbf{C}}\right)\right)_{\tau \gamma, \sigma}\right)$ and $g\left(\left(H_{3}\left(\mathbf{K}^{\mathbf{C}}\right)\right)_{\tau \gamma,-\sigma}\right)$ are given as

$$
\begin{aligned}
& \check{k}\left(\begin{array}{cccc}
\frac{1}{2}\left(\xi_{1}+\xi_{2}+\xi_{3}\right) & -a_{1} & 0 & 0 \\
-\bar{a}_{1} & \frac{1}{2}\left(\xi_{1}-\xi_{2}-\xi_{3}\right) & 0 & 0 \\
0 & 0 & \frac{1}{2}\left(-\xi_{1}+\xi_{2}-\xi_{3}\right) & m_{1} \\
0 & 0 & \bar{m}_{1} & \frac{1}{2}\left(-\xi_{1}-\xi_{2}+\xi_{3}\right)
\end{array}\right) \check{k}^{-1} \\
& =\left(\begin{array}{cccc}
\frac{1}{2}\left(\xi_{1}-\xi_{2}-\xi_{3}\right) & -\bar{a}_{1} & 0 & 0 \\
-a_{1} & \frac{1}{2}\left(\xi_{1}+\xi_{2}+\xi_{3}\right) & 0 & 0 \\
0 & 0 & \frac{1}{2}\left(-\xi_{1}-\xi_{2}+\xi_{3}\right) & -\bar{m}_{1} \\
0 & 0 & -m_{1} & -\frac{1}{2}\left(-\xi_{1}+\xi_{2}-\xi_{3}\right)
\end{array}\right) \text {, } \\
& \check{k}\left(\begin{array}{cccc}
0 & 0 & -a_{2} & -a_{3} \\
0 & 0 & m_{3} & \bar{m}_{2} \\
-\bar{a}_{2} & \bar{m}_{3} & 0 & 0 \\
-\bar{a}_{3} & m_{2} & 0 & 0
\end{array}\right) \check{k}^{-1}=\left(\begin{array}{cccc}
0 & 0 & -\bar{m}_{2} & m_{3} \\
0 & 0 & a_{3} & -a_{2} \\
-m_{2} & \bar{a}_{3} & 0 & 0 \\
\bar{m}_{3} & -\bar{a}_{2} & 0 & 0
\end{array}\right)
\end{aligned}
$$

Taking $\left(H_{3}\left(\mathbf{K}^{\mathbf{C}}\right)\right)_{\tau \gamma}=\left(H_{3}\left(\mathbf{K}^{\mathbf{C}}\right)\right)_{\tau \gamma, \sigma} \oplus\left(H_{3}\left(\mathbf{K}^{\mathbf{C}}\right)\right)_{\tau \gamma,-\sigma}$ and $H_{3}\left(\mathbf{K}^{\mathbf{C}}\right)=\left(\left(H_{3}\left(\mathbf{K}^{\mathbf{C}}\right)\right)_{\tau \gamma}\right)^{\mathbf{C}}$ into account, together with the above computation, we know that any element

$$
\left(\begin{array}{ccc}
\xi_{1} & x_{3} & \bar{x}_{2} \\
\bar{x}_{3} & \xi_{2} & x_{1} \\
x_{2} & \bar{x}_{1} & \xi_{3}
\end{array}\right)=\left(\begin{array}{ccc}
\xi_{1} & m_{3}+\sqrt{-1} a_{3} \mathbf{e} & \bar{m}_{2}-\sqrt{-1} a_{2} \mathbf{e} \\
\bar{m}_{3}-\sqrt{-1} a_{3} \mathbf{e} & \xi_{3} & m_{1}+\sqrt{-1} a_{1} \mathbf{e} \\
m_{2}+\sqrt{-1} a_{2} \mathbf{e} & \bar{m}_{1}-\sqrt{-1} a_{1} \mathbf{e} & \xi_{3}
\end{array}\right)
$$


in $H_{3}\left(\mathbf{K}^{\mathbf{C}}\right)$ is mapped by the adjoint action of $\breve{k}$ to an element

$$
\begin{aligned}
& \left(\begin{array}{ccc}
\xi_{1} & a_{3}-\sqrt{-1} m_{3} \mathbf{e} & -a_{2}-\sqrt{-1} \bar{m}_{2} \mathbf{e} \\
\bar{a}_{3}+\sqrt{-1} m_{3} \mathbf{e} & -\xi_{2} & -\bar{m}_{1}+\sqrt{-1} \bar{a}_{1} \mathbf{e} \\
-\bar{a}_{2}+\sqrt{-1} \bar{m}_{2} \mathbf{e} & -m_{1}-\sqrt{-1} \bar{a}_{1} \mathbf{e} & -\xi_{3}
\end{array}\right) \\
= & \left(\begin{array}{ccc}
\xi_{1} & \sqrt{-1}\left(-\sqrt{-1} a_{3}-m_{3} \mathbf{e}\right) & -\sqrt{-1}\left(-\sqrt{-1} a_{2}+\bar{m}_{2} \mathbf{e}\right) \\
\sqrt{-1}\left(-\sqrt{-1} \bar{a}_{3}+m_{3} \mathbf{e}\right) & -\xi_{2} & -\left(\bar{m}_{1}+\sqrt{-1} \bar{a}_{1} \mathbf{e}\right) \\
-\sqrt{-1}\left(-\sqrt{-1} \bar{a}_{2}-\bar{m}_{2} \mathbf{e}\right) & -\left(m_{1}+\sqrt{-1} \bar{a}_{1} \mathbf{e}\right) & -\xi_{3}
\end{array}\right) \\
= & \alpha_{23}(\pi) \circ\left(\alpha_{1}, \alpha_{2}, \alpha_{3}\right)\left(\left(\begin{array}{lll}
\xi_{1} & x_{3} & \bar{x}_{2} \\
\bar{x}_{3} & \xi_{2} & x_{1} \\
x_{2} & \bar{x}_{1} & \xi_{3}
\end{array}\right)\right),
\end{aligned}
$$

where $\alpha_{1}, \alpha_{2}, \alpha_{3} \in S O(\mathbf{K}) \cong S O(8)$ are defined by

$$
\begin{aligned}
& \alpha_{1}\left(m_{1}+a_{1} \mathbf{e}\right):=-\left(\bar{m}_{1}-\bar{a}_{1} \mathbf{e}\right), \\
& \alpha_{2}\left(m_{2}+a_{2} \mathbf{e}\right):=-\bar{a}_{2}-\bar{m}_{2} \mathbf{e}, \\
& \alpha_{3}\left(m_{3}+a_{3} \mathbf{e}\right):=-a_{3}-m_{3} \mathbf{e} .
\end{aligned}
$$

By a simple computation, we have

$$
\alpha_{1}\left(m_{1}+a_{1} \mathbf{e}\right) \alpha_{2}\left(m_{2}+a_{2} \mathbf{e}\right)=\overline{\alpha_{3}\left(\overline{\left(m_{1}+a_{1} \mathbf{e}\right)\left(m_{2}+a_{2} \mathbf{e}\right)}\right)} .
$$

Hence, $\left(\alpha_{1}, \alpha_{2}, \alpha_{3}\right) \in \operatorname{Spin}(8)$. Notice that

$$
\begin{aligned}
& \alpha_{23}(\pi)\left(\alpha_{1}, \alpha_{2}, \alpha_{3}\right)\left(u_{2}\right)=\alpha_{23}\left(\alpha_{2}\left(u_{2}\right)\right)=\alpha_{23}(\pi)\left(-\mathbf{e} u_{2}\right)=\sqrt{-1} \mathbf{e} u_{2}, \\
& \alpha_{23}(\pi)\left(\alpha_{1}, \alpha_{2}, \alpha_{3}\right)\left(\sqrt{-1} \mathbf{e} u_{2}\right)=\alpha_{23}(\pi)\left(\alpha_{2}\left(-\sqrt{-1} \mathbf{e} u_{2}\right)\right)=\alpha_{23}(\pi)\left(\sqrt{-1} u_{2}\right)=-u_{2} .
\end{aligned}
$$

It follows that

$$
\alpha_{23}(\pi)\left(\alpha_{1}, \alpha_{2}, \alpha_{3}\right) \in \operatorname{Spin}(2) \cdot \operatorname{Spin}(8) \subset(U(1) \times(\operatorname{Spin}(2) \cdot \operatorname{Spin}(8))) / \mathbf{Z}_{4}=K_{2}
$$

induces a linear isometry of the maximal abelian subspace $\mathfrak{a}$ of order 4 which is a $\pi / 2$-rotation of $\mathfrak{a}$, we obtain

$$
\alpha_{23}(\pi)\left(\alpha_{1}, \alpha_{2}, \alpha_{3}\right) \in K_{[\mathfrak{a}]}
$$

and it is a generator of $K_{[\mathfrak{a}]} / K_{0} \cong \mathbf{Z}_{4}$.

5.9. Description of the Casimir operator. Define $\langle u, v\rangle_{\mathfrak{u}}:=-\operatorname{tr}(u v)$ for each $u, v \in$ $\mathfrak{e}_{6} \subset \mathfrak{g l}\left(H_{3}(\mathbf{K})^{\mathbf{C}}\right)$. Now the positive restricted root system is $\Sigma^{+}(U, K)=\left\{2 \xi_{1}, 2 \xi_{2}, \xi_{1}+\right.$ $\left.\xi_{2}, \xi_{1}-\xi_{2}, \xi_{1}, \xi_{2}\right\}$ and

$$
H_{\xi_{1}}=\frac{1}{12}\left(D\left(\bar{u}_{2}\right)+\sqrt{-1} R\left(c_{4} u_{2}\right)\right), \quad H_{\xi_{2}}=\frac{1}{12}\left(D\left(\bar{u}_{2}\right)-\sqrt{-1} R\left(c_{4} u_{2}\right)\right) .
$$

The square lengths of the restricted roots with respect to $\langle,\rangle_{\mathfrak{u}}$ are given by

$$
\|\gamma\|_{\mathfrak{u}}^{2}=\frac{1}{3}, \frac{1}{6} \text { or } \frac{1}{12} \text {. }
$$

Then the Casimir operator $\mathcal{C}_{L}$ with respect to the induced metric $\mathcal{G}^{*} g_{Q_{30}(\mathbf{C})}^{\text {std }}$ can be expressed as

$$
\mathcal{C}_{L}=12 \mathcal{C}_{K / K_{0}}-6 \mathcal{C}_{K_{2} / K_{0}}-3 \mathcal{C}_{K_{1} / K_{0}}
$$


where $\mathcal{C}_{K / K_{0}}, \mathcal{C}_{K_{2} / K_{0}}$ and $\mathcal{C}_{K_{1} / K_{0}}$ are the Casimir operators of compact homogeneous spaces $K / K_{0}, K_{2} / K_{0}$ and $K_{1} / K_{0}$ relative to the $K$-invariant Riemannian metric induced from an inner product $\langle,\rangle_{\mathfrak{u}}$ of $E_{6}$.

5.10. Descriptions of $D(K), D\left(K_{2}\right), D\left(K_{1}\right)$ and $D\left(K_{0}\right)$. A maximal torus $\tilde{T}^{5}$ of $\operatorname{Spin}(10)$ can be given by

$$
\begin{aligned}
\tilde{T}^{5}= & \left\{\tilde{t}=\left(\cos \theta_{1}-e_{1} e_{2} \sin \theta_{1}\right) \cdot\left(\cos \theta_{2}-e_{3} e_{4} \sin \theta_{2}\right) \cdot\left(\cos \theta_{3}-e_{5} e_{6} \sin \theta_{3}\right)\right. \\
& \left.\cdot\left(\cos \theta_{4}-e_{7} e_{8} \sin \theta_{4}\right) \cdot\left(\cos \theta_{5}-e_{9} e_{10} \sin \theta_{5}\right) \mid \theta_{i} \in \mathbf{R}(i=1,2,3,4,5)\right\} .
\end{aligned}
$$

Under the standard universal $\mathbf{Z}_{2}$-covering map $p: \operatorname{Spin}(10) \rightarrow S O(10)$ defined by

$$
(p(\alpha)) \mathbf{x}:=\alpha \cdot \mathbf{x} \cdot{ }^{t} \alpha \in \mathbf{R}^{10} \subset C l\left(\mathbf{R}^{10}\right)
$$

for each $\alpha \in \operatorname{Spin}(10)$ and each $\mathbf{x} \in \mathbf{R}^{10}$, an element of the maximal torus $\tilde{T}^{5}$ of $\operatorname{Spin}(10)$ is mapped to an element in the maximal torus $T^{5}$ of $S O(10)$ as

$$
\begin{aligned}
& \tilde{T}^{5} \ni\left(\cos \theta_{1}-e_{1} e_{2} \sin \theta_{1}\right) \cdot\left(\cos \theta_{2}-e_{3} e_{4} \sin \theta_{2}\right) \cdot\left(\cos \theta_{3}-e_{5} e_{6} \sin \theta_{3}\right) \\
& \cdot\left(\cos \theta_{4}-e_{7} e_{8} \sin \theta_{4}\right) \cdot\left(\cos \theta_{5}-e_{9} e_{10} \sin \theta_{5}\right) \\
& \longmapsto\left(\begin{array}{ccc}
\left(\begin{array}{ccc}
\cos 2 \theta_{1} & -\sin 2 \theta_{1} \\
\sin 2 \theta_{1} & \cos 2 \theta_{1}
\end{array}\right) & 0 & 0 \\
0 & \ldots & 0 \\
0 & 0 & \left(\begin{array}{cc}
\cos 2 \theta_{5} & -\sin 2 \theta_{5} \\
\sin 2 \theta_{5} & \cos 2 \theta_{5}
\end{array}\right)
\end{array}\right) \in T^{5} .
\end{aligned}
$$

Hence we have the exponential map as follows:

$$
\begin{aligned}
& \exp : \tilde{\mathfrak{t}}=\mathfrak{t}=\left\{\left(\theta_{1}, \theta_{2}, \theta_{3}, \theta_{4}, \theta_{5}\right) \mid \theta_{i} \in \mathbf{R}(i=1,2,3,4,5)\right\} \\
& \longrightarrow \tilde{T}=\{\left(\cos \left(\theta_{1} / 2\right)-e_{1} e_{2} \sin \left(\theta_{1} / 2\right)\right) \cdot\left(\cos \left(\theta_{2} / 2\right)-e_{3} e_{4} \sin \left(\theta_{2} / 2\right)\right) \\
& \cdot\left(\cos \left(\theta_{3} / 2\right)-e_{5} e_{6} \sin \left(\theta_{3} / 2\right)\right) \cdot\left(\cos \left(\theta_{4} / 2\right)-e_{7} e_{8} \sin \left(\theta_{4} / 2\right)\right) \\
& \cdot\left(\cos \left(\theta_{5} / 2\right)-e_{9} e_{10} \sin \left(\theta_{5} / 2\right)\right) \\
&\left.\mid \theta_{i} \in \mathbf{R}(i=1,2,3,4,5)\right\} \subset \operatorname{Spin}(10) .
\end{aligned}
$$

Thus

$$
\begin{aligned}
& \Gamma(\operatorname{Spin}(10))=\left\{\xi=\left(\theta_{1}, \theta_{2}, \theta_{3}, \theta_{4}, \theta_{5}\right) \in \tilde{\mathfrak{t}} \mid \exp (\xi)=e\right\} \\
= & \left\{\xi=2 \pi\left(k_{1}, k_{2}, k_{3}, k_{4}, k_{5}\right) \mid k_{i} \in \mathbf{Z}(i=1,2,3,4,5), \sum_{i=1}^{5} k_{i} \in 2 \mathbf{Z}\right\} \subset \Gamma(S O(10)) .
\end{aligned}
$$

Denote by $y_{i}(i=1, \ldots, 5)$ a linear function $y_{i}: \tilde{\mathfrak{t}} \ni \tilde{t} \mapsto \theta_{i} \in \mathbf{R}$. Then

$$
\begin{aligned}
D(\operatorname{Spin}(10))= & \left\{\Lambda=p_{1} y_{1}+p_{2} y_{2}+p_{3} y_{3}+p_{4} y_{4}+p_{5} y_{5} \in \mathfrak{t}^{*}\right. \\
& \mid\left(p_{1}, \ldots, p_{5}\right) \in \mathbf{Z}^{5}+\varepsilon(1,1,1,1,1), \text { where } \varepsilon=0 \text { or } \frac{1}{2}, \\
& \left.p_{1} \geq p_{2} \geq p_{3} \geq p_{4} \geq\left|p_{5}\right|\right\} \supset D(S O(10)) .
\end{aligned}
$$


A maximal torus $T_{K}$ of $K=(U(1) \times \operatorname{Spin}(10)) / \mathbf{Z}_{4}$ can be given as follows:

$$
\begin{aligned}
T_{K}= & \left\{\left(e^{\sqrt{-1} \theta_{0}},\left(\cos \frac{\theta_{1}}{2}-e_{1} e_{2} \sin \frac{\theta_{1}}{2}\right)\left(\cos \frac{\theta_{2}}{2}-e_{3} e_{4} \sin \frac{\theta_{2}}{2}\right)\right.\right. \\
& \left.\left(\cos \frac{\theta_{3}}{2}-e_{5} e_{6} \sin \frac{\theta_{3}}{2}\right)\left(\cos \frac{\theta_{4}}{2}-e_{7} e_{8} \sin \frac{\theta_{4}}{2}\right)\left(\cos \frac{\theta_{5}}{2}-e_{9} e_{10} \sin \frac{\theta_{5}}{2}\right)\right) \\
& \left.\mid \theta_{0}, \ldots, \theta_{5} \in \mathbf{R}\right\} / \mathbf{Z}_{4},
\end{aligned}
$$

where $t_{0}=2 \theta_{0}, t_{1}=\theta_{1}, U(1)=\left\{\exp \left(t_{0} \sqrt{-1} R\left(2 e_{1}-e_{2}-e_{3}\right)\right) \mid t_{0} \in \mathbf{R}\right\}, \operatorname{Spin}(2)=$ $\left\{\exp \left(t_{1} \sqrt{-1} R\left(e_{2}-e_{3}\right)\right) \mid t_{1} \in \mathbf{R}\right\}$ and

$$
\mathbf{Z}_{4}:=\left\{(1,1),(-1,-1),\left(\sqrt{-1},-e_{1} e_{2} \cdots e_{10}\right),\left(-\sqrt{-1}, e_{1} e_{2} \cdots e_{10}\right)\right\}
$$

The corresponding maximal abelian subalgebra $\mathfrak{t}$ of $\mathfrak{k}$ is

$$
\mathfrak{t}=\left\{\left(\theta_{0}, \theta_{1}, \theta_{2}, \theta_{3}, \theta_{4}, \theta_{5}\right) \mid \theta_{i} \in \mathbf{R}(i=0,1,2,3,4,5)\right\} .
$$

Then

$$
\begin{aligned}
\Gamma(K)= & \left\{\xi=2 \pi\left(\frac{k_{0}}{2}, k_{1}, k_{2}, k_{3}, k_{4}, k_{5}\right)+\pi \varepsilon\left(\frac{1}{2}, 1,1,1,1,1\right)\right. \\
& \left.\mid k_{0}, k_{1}, k_{2}, k_{3}, k_{4}, k_{5} \in \mathbf{Z}, \varepsilon=0 \text { or } 1, \sum_{\alpha=0}^{5} k_{\alpha} \in 2 \mathbf{Z}\right\}, \\
D(K)= & D\left((U(1) \times \operatorname{Spin}(10)) / \mathbf{Z}_{4}\right) \\
= & \left\{\Lambda=p_{0} y_{0}+p_{1} y_{1}+p_{2} y_{2}+p_{3} y_{3}+p_{4} y_{4}+p_{5} y_{5} \in \mathfrak{t}^{*}\right. \\
& \mid \frac{1}{2} p_{0}+p_{1}+p_{2}+p_{3}+p_{4}+p_{5} \in 2 \mathbf{Z}, p_{0} \in \mathbf{Z}, \\
& \left(p_{1}, p_{2}, p_{3}, p_{4}, p_{5}\right) \in \mathbf{Z}^{5}+\varepsilon(1,1,1,1,1), \varepsilon=0 \text { or } \frac{1}{2}, \\
& \left.p_{1} \geq p_{2} \geq p_{3} \geq p_{4} \geq\left|p_{5}\right|\right\} .
\end{aligned}
$$


Since $T_{K}$ is also a maximal torus of $K_{2}=(U(1) \times(\operatorname{Spin}(2) \cdot \operatorname{Spin}(8))) / \mathbf{Z}_{4} \subset K$, $\Gamma\left(K_{2}\right)=\Gamma(K)$ and

$$
\begin{aligned}
& D\left(K_{2}\right)=D\left((U(1) \times \operatorname{Spin}(2) \cdot \operatorname{Spin}(8)) / \mathbf{Z}_{4}\right) \\
& =\left\{\Lambda=p_{0} y_{0}+p_{1} y_{1}+p_{2} y_{2}+p_{3} y_{3}+p_{4} y_{4}+p_{5} y_{5} \in \mathfrak{t}^{*}\right. \\
& \mid \frac{1}{2} p_{0}+p_{1}+p_{2}+p_{3}+p_{4}+p_{5} \in 2 \mathbf{Z}, p_{0} \in \mathbf{Z}, \\
& \left(p_{1}, p_{2}, p_{3}, p_{4}, p_{5}\right) \in \mathbf{Z}^{5}+\varepsilon(1,1,1,1,1), \varepsilon=0 \text { or } \frac{1}{2} \text {, } \\
& \left.p_{2} \geq p_{3} \geq p_{4} \geq\left|p_{5}\right|\right\} \text {. }
\end{aligned}
$$

On the other hand, $K_{2}=\left(S^{1} \times(\operatorname{Spin}(2) \cdot \operatorname{Spin}(8))\right) / \mathbf{Z}_{4}$, where

$$
\begin{aligned}
S^{1} & =\left\{\exp \left(\hat{t}_{0} \sqrt{-1} R\left(-e_{1}+2 e_{2}-e_{3}\right)\right) \mid \hat{t}_{0} \in \mathbf{R}\right\}, \\
\operatorname{Spin}(2) & =\left\{\exp \left(\hat{t}_{1} \sqrt{-1} R\left(e_{3}-e_{1}\right)\right) \mid \hat{t}_{1} \in \mathbf{R}\right\}
\end{aligned}
$$

and here $\operatorname{Spin}(2) \cdot \operatorname{Spin}(8) \subset\left(E_{6}\right)_{e_{2}} \cong \operatorname{Spin}(10)$. Since

$$
\begin{aligned}
& \exp \left(t_{0} \sqrt{-1} R\left(2 e_{1}-e_{2}-e_{3}\right)\right) \cdot \exp \left(t_{1} \sqrt{-1} R\left(e_{2}-e_{3}\right)\right) \\
= & \exp \left(-\frac{t_{0}-t_{1}}{2} \sqrt{-1} R\left(-e_{1}+2 e_{2}-e_{3}\right)\right) \cdot \exp \left(-\frac{3 t_{0}+t_{1}}{2} \sqrt{-1} R\left(e_{3}-e_{1}\right)\right),
\end{aligned}
$$

one can take $\hat{t}_{0}=-\frac{t_{0}-t_{1}}{2}, \hat{t}_{1}=-\frac{3 t_{0}+t_{1}}{2}$ such that the maximal torus $T_{K_{2}}=T_{K}$ of $K_{2}$ can also be described as

$$
\begin{gathered}
\hat{T}_{K_{2}}=T_{K_{2}}=T_{K}=\left\{\hat{t}=\left(e^{\sqrt{-1} \hat{\theta}_{0}},\left(\cos \frac{\hat{\theta}_{1}}{2}-e_{1} e_{2} \sin \frac{\hat{\theta}_{1}}{2}\right)\left(\cos \frac{\hat{\theta}_{2}}{2}-e_{3} e_{4} \sin \frac{\hat{\theta}_{2}}{2}\right)\right.\right. \\
\left.\left(\cos \frac{\hat{\theta}_{3}}{2}-e_{5} e_{6} \sin \frac{\hat{\theta}_{3}}{2}\right)\left(\cos \frac{\hat{\theta}_{4}}{2}-e_{7} e_{8} \sin \frac{\hat{\theta}_{4}}{2}\right)\left(\cos \frac{\hat{\theta}_{5}}{2}-e_{9} e_{10} \sin \frac{\hat{\theta}_{5}}{2}\right)\right) \\
\left.\mid \hat{\theta}_{0}, \ldots, \hat{\theta}_{5} \in \mathbf{R}\right\} / \mathbf{Z}_{4},
\end{gathered}
$$

where $\hat{\theta}_{0}=\hat{t}_{0} / 2, \hat{\theta}_{1}=\hat{t}_{1}$. Taking account of the triality of $\operatorname{Spin}(8)=\left(E_{6}\right)_{e_{1}, e_{2}, e_{3}} \subset\left(E_{6}\right)_{e_{1}} \cong$ $\left(E_{6}\right)_{e_{2}} \cong \operatorname{Spin}(10)$, we choose a new basis $\hat{y}_{i}: \hat{t} \mapsto \hat{\theta}_{i}$ for $\mathfrak{t}^{*}$ satisfying

$$
\begin{aligned}
& \hat{y}_{0}=-\frac{1}{2} y_{0}+\frac{1}{4} y_{1}, \quad \hat{y}_{1}=-3 y_{0}-\frac{1}{2} y_{1}, \quad \hat{y}_{2}:=\frac{1}{2}\left(y_{2}+y_{3}+y_{4}+y_{5}\right), \\
& \hat{y}_{3}:=\frac{1}{2}\left(y_{2}+y_{3}-y_{4}-y_{5}\right), \quad \hat{y}_{4}:=\frac{1}{2}\left(y_{2}-y_{3}+y_{4}-y_{5}\right), \\
& \hat{y}_{5}:=\frac{1}{2}\left(-y_{2}+y_{3}+y_{4}-y_{5}\right) .
\end{aligned}
$$


Thus any $\Lambda=p_{0} y_{0}+p_{1} y_{1}+p_{2} y_{2}+p_{3} y_{3}+p_{4} y_{4}+p_{5} y_{5} \in D\left(K_{2}\right)$ can also be written as $\Lambda=\hat{p}_{0} \hat{y}_{0}+\hat{p}_{1} \hat{y}_{1}+\hat{p}_{2} \hat{y}_{2}+\hat{p}_{3} \hat{y}_{3}+\hat{p}_{4} \hat{y}_{4}+\hat{p}_{5} \hat{y}_{5}$, where

$$
\begin{aligned}
& \hat{p}_{0}=-\frac{1}{2} p_{0}+3 p_{1}, \quad \hat{p}_{1}=-\frac{1}{4} p_{0}-\frac{1}{2} p_{1}, \quad \hat{p}_{2}=\frac{1}{2}\left(p_{2}+p_{3}+p_{4}+p_{5}\right), \\
& \hat{p}_{3}=\frac{1}{2}\left(p_{2}+p_{3}-p_{4}-p_{5}\right), \quad \hat{p}_{4}=\frac{1}{2}\left(p_{2}-p_{3}+p_{4}-p_{5}\right), \\
& \hat{p}_{5}=\frac{1}{2}\left(-p_{2}+p_{3}+p_{4}-p_{5}\right) .
\end{aligned}
$$

Thus $D\left(K_{2}\right)$ has the following another expression:

$$
\begin{aligned}
D\left(K_{2}\right)= & D\left(\left(S^{1} \times \operatorname{Spin}(2) \cdot \operatorname{Spin}(8)\right) / \mathbf{Z}_{4}\right) \\
= & \left\{\Lambda=\hat{p}_{0} \hat{y}_{0}+\hat{p}_{1} \hat{y}_{1}+\hat{p}_{2} \hat{y}_{2}+\hat{p}_{3} \hat{y}_{3}+\hat{p}_{4} \hat{y}_{4}+\hat{p}_{5} \hat{y}_{5} \in \mathfrak{t}^{*}\right. \\
& \mid \frac{1}{2} \hat{p}_{0}+\hat{p}_{1}+\hat{p}_{2}+\hat{p}_{3}+\hat{p}_{4}+\hat{p}_{5} \in 2 \mathbf{Z}, \hat{p}_{0} \in \mathbf{Z}, \\
& \left(\hat{p}_{1}, \hat{p}_{2}, \hat{p}_{3}, \hat{p}_{4}, \hat{p}_{5}\right) \in \mathbf{Z}^{5}+\varepsilon(1,1,1,1,1), \varepsilon=0 \text { or } \frac{1}{2}, \\
& \left.\hat{p}_{2} \geq \hat{p}_{3} \geq \hat{p}_{4} \geq\left|\hat{p}_{5}\right|\right\} .
\end{aligned}
$$

Notice that the subgroup $K_{1}=\left(S^{1} \times(\operatorname{Spin}(2) \cdot(\operatorname{Spin}(2) \cdot \operatorname{Spin}(6)))\right) / \mathbf{Z}_{4}$ also has the same maximal torus $T_{K_{1}}=\hat{T}_{K_{2}}=T_{K_{2}}=T_{K}$ and the corresponding maximal abelian subalgebra $\mathfrak{t}_{\mathfrak{k}_{1}}$ of $\mathfrak{k}_{1}$ is

$$
\mathfrak{t}_{\mathfrak{k}_{1}}=\hat{\mathfrak{t}}_{\mathfrak{k}_{2}}=\left\{\left(\hat{\theta}_{0}, \hat{\theta}_{1}, \hat{\theta}_{2}, \hat{\theta}_{3}, \hat{\theta}_{4}, \hat{\theta}_{5}\right) \mid \hat{\theta}_{i} \in \mathbf{R}(i=0,1,2,3,4,5)\right\}=\mathfrak{t}_{\mathfrak{k}_{2}}=\mathfrak{t},
$$

we get

$$
\begin{aligned}
D\left(K_{1}\right)=\{ & \left\{\Lambda=\hat{p}_{0} \hat{y}_{0}+\hat{p}_{1} \hat{y}_{1}+\hat{p}_{2} \hat{y}_{2}+\hat{p}_{3} \hat{y}_{3}+\hat{p}_{4} \hat{y}_{4}+\hat{p}_{5} \hat{y}_{5} \in \mathfrak{t}_{\mathfrak{k}_{1}}^{*}=\mathfrak{t}^{*}\right. \\
& \mid \frac{1}{2} \hat{p}_{0}+\hat{p}_{1}+\hat{p}_{2}+\hat{p}_{3}+\hat{p}_{4}+\hat{p}_{5} \in 2 \mathbf{Z}, \hat{p}_{0} \in \mathbf{Z}, \\
& \left(\hat{p}_{1}, \hat{p}_{2}, \hat{p}_{3}, \hat{p}_{4}, \hat{p}_{5}\right) \in \mathbf{Z}^{5}+\varepsilon(1,1,1,1,1), \varepsilon=0 \text { or } \frac{1}{2}, \\
& \left.\hat{p}_{3} \geq \hat{p}_{4} \geq\left|\hat{p}_{5}\right|\right\} .
\end{aligned}
$$

Finally, the maximal torus of $K_{0}=\left(S^{1} \times \operatorname{Spin}(6)\right) / \mathbf{Z}_{2}$ is given as follows:

$$
\begin{aligned}
T_{K_{0}}=\{ & \left(e^{\sqrt{-1} \hat{\theta}_{0}},\left(\cos \frac{\hat{\theta}_{3}}{2}-e_{5} e_{6} \sin \frac{\hat{\theta}_{3}}{2}\right)\left(\cos \frac{\hat{\theta}_{4}}{2}-e_{7} e_{8} \sin \frac{\hat{\theta}_{4}}{2}\right)\right. \\
& \left.\left.\left(\cos \frac{\hat{\theta}_{5}}{2}-e_{9} e_{10} \sin \frac{\hat{\theta}_{5}}{2}\right)\right) \mid \hat{\theta}_{i} \in \mathbf{R}(i=0,3,4,5)\right\} / \mathbf{Z}_{2} \subset \hat{T}_{K_{2}}=T_{K}
\end{aligned}
$$


and the corresponding maximal abelian subalgebra of $\mathfrak{k}_{0}$ is

$$
\mathfrak{t}_{\mathfrak{k}_{0}}=\left\{\left(\hat{\theta}_{0}, 0,0, \hat{\theta}_{3}, \hat{\theta}_{4}, \hat{\theta}_{5}\right) \mid \hat{\theta}_{i} \in \mathbf{R}(i=0,3,4,5)\right\} \subset \mathfrak{t}_{\mathfrak{k}_{2}}=\mathfrak{t} .
$$

Then

$$
\begin{aligned}
& D\left(K_{0}\right)=\left\{\Lambda=\hat{q}_{0} \hat{y}_{0}+\hat{q}_{3} \hat{y}_{3}+\hat{q}_{4} \hat{y}_{4}+\hat{q}_{5} \hat{y}_{5} \in \mathfrak{t}_{\mathfrak{k}_{0}}^{*}\right. \\
& \mid \frac{1}{2} \hat{q}_{0}+\hat{q}_{3}+\hat{q}_{4}+\hat{q}_{5} \in 2 \mathbf{Z}, \hat{q}_{0} \in \mathbf{Z}, \\
& \left(\hat{q}_{3}, \hat{q}_{4}, \hat{q}_{5}\right) \in \mathbf{Z}^{3}+\varepsilon(1,1,1), \varepsilon=0 \text { or } \frac{1}{2}, \\
& \left.\hat{q}_{3} \geq \hat{q}_{4} \geq\left|\hat{q}_{5}\right|\right\} \text {. }
\end{aligned}
$$

5.11. Branching laws. Based on the branching laws of $(S O(2 n+2), S O(2) \times$ $S O(2 n)$ ) obtained by Tsukamoto ([24]), we formulate the following branching laws.

LEMMA 5.3 (Branching Law of (Spin(10), Spin(2) - Spin(8))). For each

$$
\Lambda=p_{1} y_{1}+p_{2} y_{2}+p_{3} y_{3}+p_{4} y_{4}+\delta p_{5} y_{5} \in D(\operatorname{Spin}(10)),
$$

with $\delta=1$ or -1 and

$$
\begin{aligned}
& \left(p_{1}, p_{2}, p_{3}, p_{4}, p_{5}\right) \in \mathbf{Z}^{5}+\varepsilon(1,1,1,1,1), \varepsilon=0 \text { or } \frac{1}{2}, \\
& p_{1} \geq p_{2} \geq p_{3} \geq p_{4} \geq p_{5} \geq 0,
\end{aligned}
$$

$V_{\Lambda}$ contains an irreducible Spin(2) $\cdot \operatorname{Spin}(8)$-module with the highest weight

$$
\Lambda^{\prime}=q_{1} y_{1}+q_{2} y_{2}+q_{3} y_{3}+q_{4} y_{4}+\delta^{\prime} q_{5} y_{5} \in D(\operatorname{Spin}(2) \cdot \operatorname{Spin}(8))
$$

with $\delta^{\prime}=1$ or -1 and

$$
\begin{aligned}
& \left(q_{1}, q_{2}, q_{3}, q_{4}, q_{5}\right) \in \mathbf{Z}^{5}+\varepsilon(1,1,1,1,1), \varepsilon=0 \text { or } \frac{1}{2}, \\
& q_{2} \geq q_{3} \geq q_{4} \geq q_{5} \geq 0,
\end{aligned}
$$

if and only if $\Lambda^{\prime}$ satisfies the following conditions:

(1)

$$
\begin{aligned}
& p_{1}+1>q_{2}>p_{3}-1, \\
& p_{2}+1>q_{3}>p_{4}-1, \\
& p_{3}+1>q_{4}>p_{5}-1, \\
& p_{4}+1>q_{5} \geq 0 .
\end{aligned}
$$

(2) The coefficient of $X^{q_{1}}$ in the following power series expansion in $X$ of

$$
X^{\delta \delta^{\prime} \ell_{5}}\left(\prod_{i=1}^{4} \frac{X^{\ell_{i}+1}-X^{-\ell_{i}-1}}{X-X^{-1}}\right)
$$


does not vanish. Here

$$
\begin{aligned}
& \ell_{1}:=p_{1}-\max \left\{p_{2}, q_{2}\right\}, \\
& \ell_{2}:=\min \left\{p_{2}, q_{2}\right\}-\max \left\{p_{3}, q_{3}\right\}, \\
& \ell_{3}:=\min \left\{p_{3}, q_{3}\right\}-\max \left\{p_{4}, q_{4}\right\}, \\
& \ell_{4}:=\min \left\{p_{4}, q_{4}\right\}-\max \left\{p_{5}, q_{5}\right\}, \\
& \ell_{5}:=\min \left\{p_{5}, q_{5}\right\} .
\end{aligned}
$$

Moreover its multiplicity is equal to the coefficient of $X^{q_{1}}$.

LEMMA 5.4 (Branching Law of (Spin(8), Spin(2) - Spin(6))). For each

$$
\Lambda=p_{2} y_{2}+p_{3} y_{3}+p_{4} y_{4}+\delta p_{5} y_{5} \in D(\operatorname{Spin}(8)),
$$

with $\delta=1$ or -1 and

$$
\begin{aligned}
& \left(p_{2}, p_{3}, p_{4}, p_{5}\right) \in \mathbf{Z}^{4}+\varepsilon(1,1,1,1), \varepsilon=0 \text { or } \frac{1}{2}, \\
& p_{2} \geq p_{3} \geq p_{4} \geq p_{5} \geq 0,
\end{aligned}
$$

$V_{\Lambda}$ contains an irreducible Spin(2) $\cdot$ Spin(6)-module with the highest weight

$$
\Lambda^{\prime}=q_{2} y_{2}+q_{3} y_{3}+q_{4} y_{4}+\delta^{\prime} p_{5} y_{5} \in D(\operatorname{Spin}(2) \cdot \operatorname{Spin}(6))
$$

with $\delta^{\prime}=1$ or -1 and

$$
\begin{aligned}
& \left(q_{2}, q_{3}, q_{4}, q_{5}\right) \in \mathbf{Z}^{4}+\varepsilon(1,1,1,1), \varepsilon=0 \text { or } \frac{1}{2}, \\
& q_{3} \geq q_{4} \geq q_{5} \geq 0,
\end{aligned}
$$

if and only if $\Lambda^{\prime}$ satisfies the following conditions:

(1)

$$
\begin{aligned}
& p_{2}+1>q_{3}>p_{4}-1, \\
& p_{3}+1>q_{4}>p_{5}-1, \\
& p_{4}+1>q_{5} \geq 0 .
\end{aligned}
$$

(2) The coefficient of $X^{q_{2}}$

$$
X^{\delta \delta^{\prime} \ell_{5}}\left(\prod_{i=2}^{4} \frac{X^{\ell_{i}+1}-X^{-\ell_{i}-1}}{X-X^{-1}}\right)
$$

does not vanish. Here

$$
\begin{aligned}
\ell_{2} & :=p_{2}-\max \left\{p_{3}, q_{3}\right\}, \\
\ell_{3} & :=\min \left\{p_{3}, q_{3}\right\}-\max \left\{p_{4}, q_{4}\right\}, \\
\ell_{4} & :=\min \left\{p_{4}, q_{4}\right\}-\max \left\{p_{5}, q_{5}\right\}, \\
\ell_{5} & :=\min \left\{p_{5}, q_{5}\right\} .
\end{aligned}
$$

Moreover its multiplicity is equal to the coefficient of $X^{q_{2}}$. 
5.12. Description of $D\left(K, K_{0}\right)$. Let

$$
\begin{aligned}
\Lambda & =p_{0} y_{0}+p_{1} y_{1}+p_{2} y_{2}+p_{3} y_{3}+p_{4} y_{4}+\varepsilon p_{5} y_{5} \in D(K), \\
\Lambda^{\prime} & =p_{0}^{\prime} y_{0}+p_{1}^{\prime} y_{1}+p_{2}^{\prime} y_{2}+p_{3}^{\prime} y_{3}+p_{4}^{\prime} y_{4}+\varepsilon^{\prime} p_{5}^{\prime} y_{5} \\
& =\hat{p}_{0}^{\prime} \hat{y}_{0}+\hat{p}_{1}^{\prime} \hat{y}_{1}+\hat{p}_{2}^{\prime} \hat{y}_{2}+\hat{p}_{3}^{\prime} \hat{y}_{3}+\hat{p}_{4}^{\prime} \hat{y}_{4}+\hat{\varepsilon}^{\prime} \hat{p}_{5}^{\prime} \hat{y}_{5} \in D\left(K_{2}\right), \\
\Lambda^{\prime \prime} & =\hat{p}_{0}^{\prime \prime} \hat{y}_{0}+\hat{p}_{1}^{\prime \prime} \hat{y}_{1}+\hat{p}_{2}^{\prime \prime} \hat{y}_{2}+\hat{p}_{3}^{\prime \prime} \hat{y}_{3}+\hat{p}_{4}^{\prime \prime} \hat{y}_{4}+\hat{\varepsilon}^{\prime \prime} \hat{p}_{5}^{\prime \prime} \hat{y}_{5} \in D\left(K_{1}\right), \\
\Lambda^{\prime \prime \prime} & =\hat{p}_{0}^{\prime \prime \prime} \hat{y}_{0}+\hat{p}_{3}^{\prime \prime \prime} \hat{y}_{3}+\hat{p}_{4}^{\prime \prime \prime} \hat{y}_{4}+\hat{\varepsilon}^{\prime \prime \prime} \hat{p}_{5}^{\prime \prime \prime} \hat{y}_{5} \in D\left(K_{0}\right) .
\end{aligned}
$$

Assume that the corresponding representation spaces satisfy

$$
V_{\Lambda} \supset W_{\Lambda^{\prime}} \supset U_{\Lambda^{\prime \prime}}=U_{\Lambda^{\prime \prime \prime}} \neq\{0\} .
$$

Suppose that $U_{\Lambda^{\prime \prime \prime}} \neq\{0\}$ is a trivial representation of $K_{0}$, that is, $\Lambda^{\prime \prime \prime}=0$. Then we have

$$
\hat{p}_{0}^{\prime \prime \prime}=\hat{p}_{0}^{\prime \prime}=0, \quad \hat{p}_{3}^{\prime \prime \prime}=\hat{p}_{3}^{\prime \prime}=0, \quad \hat{p}_{4}^{\prime \prime \prime}=\hat{p}_{4}^{\prime \prime}=0, \quad \hat{p}_{5}^{\prime \prime \prime}=\hat{p}_{5}^{\prime \prime}=0 .
$$

Thus $\Lambda^{\prime \prime}=\hat{p}_{1}^{\prime \prime} \hat{y}_{1}+\hat{p}_{2}^{\prime \prime} \hat{y}_{2} \in D\left(K_{1}\right)$ with $\hat{p}_{1}^{\prime \prime}, \hat{p}_{2}^{\prime \prime} \in \mathbf{Z}, \hat{p}_{1}^{\prime \prime}+\hat{p}_{2}^{\prime \prime} \in 2 \mathbf{Z}$.

By the branching law of $(\operatorname{Spin}(8), \operatorname{Spin}(2) \cdot \operatorname{Spin}(6))$, we get

$$
\begin{aligned}
& \hat{p}_{2}^{\prime} \geq \hat{p}_{3}^{\prime \prime}=0 \geq \hat{p}_{4}^{\prime}, \\
& \hat{p}_{3}^{\prime} \geq \hat{p}_{4}^{\prime \prime}=0 \geq \hat{p}_{5}^{\prime}, \\
& \hat{p}_{4}^{\prime} \geq \hat{p}_{5}^{\prime \prime}=0 \geq 0 .
\end{aligned}
$$

Thus $\left(\hat{p}_{4}^{\prime}, \hat{p}_{5}^{\prime}\right)=(0,0)$ and $\hat{p}_{2}^{\prime} \geq 0, \hat{p}_{3}^{\prime} \geq 0$. It follows that

$$
\begin{aligned}
& \ell_{2}=\hat{p}_{2}^{\prime}-\max \left\{\hat{p}_{3}^{\prime}, \hat{p}_{3}^{\prime \prime}\right\}=\hat{p}_{2}^{\prime}-\max \left\{\hat{p}_{3}^{\prime}, 0\right\}=\hat{p}_{2}^{\prime}-\hat{p}_{3}^{\prime}, \\
& \ell_{3}=\min \left\{\hat{p}_{3}^{\prime}, \hat{p}_{3}^{\prime \prime}\right\}-\max \left\{\hat{p}_{4}^{\prime}, \hat{p}_{4}^{\prime \prime}\right\}=\min \left\{\hat{p}_{3}^{\prime}, 0\right\}-\max \{0,0\}=0-0=0, \\
& \ell_{4}=\min \left\{\hat{p}_{4}^{\prime}, \hat{p}_{4}^{\prime \prime}\right\}-\max \left\{\hat{p}_{5}^{\prime}, \hat{p}_{5}^{\prime \prime}\right\}=\min \{0,0\}-\max \{0,0\}=0-0=0, \\
& \ell_{5}=\min \left\{\hat{p}_{5}^{\prime}, \hat{p}_{5}^{\prime \prime}\right\}=\min \{0,0\}=0 .
\end{aligned}
$$

Then the coefficient of $X^{\hat{p}_{2}^{\prime \prime}}$ in the (finite) power series expansion in $X$

$$
X^{\hat{\varepsilon}^{\prime} \hat{\varepsilon}^{\prime \prime} \ell_{5}} \prod_{i=2}^{4} \frac{X^{\ell_{i}+1}-X^{-\ell_{i}-1}}{X-X^{-1}}=\frac{X^{\hat{p}_{2}^{\prime}-\hat{p}_{3}^{\prime}+1}-X^{-\left(\hat{p}_{2}^{\prime}-\hat{p}_{3}^{\prime}\right)-1}}{X-X^{-1}}
$$

is equal to its multiplicity. Hence we have

$$
-\left(\hat{p}_{2}^{\prime}-\hat{p}_{3}^{\prime}\right) \leq \hat{p}_{2}^{\prime \prime}=\hat{p}_{2}^{\prime}-\hat{p}_{3}^{\prime}-2 i \leq \hat{p}_{2}^{\prime}-\hat{p}_{3}^{\prime}
$$

for some $i \in \mathbf{Z}$ with $0 \leq i \leq \hat{p}_{2}^{\prime}-\hat{p}_{3}^{\prime}$. Moreover, $\hat{p}_{0}^{\prime}=\hat{p}_{0}^{\prime \prime}=0, \hat{p}_{1}^{\prime}=\hat{p}_{1}^{\prime \prime}$. Thus we get

$$
\Lambda^{\prime}=\hat{p}_{1}^{\prime} \hat{y}_{1}+\hat{p}_{2}^{\prime} \hat{y}_{2}+\hat{p}_{3}^{\prime} \hat{y}_{3} \in D\left(K_{2}\right)
$$

with

$$
\begin{aligned}
& \hat{p}_{1}^{\prime}=\hat{p}_{1}^{\prime \prime}, \hat{p}_{2}^{\prime}, \hat{p}_{3}^{\prime} \in \mathbf{Z}, \quad \hat{p}_{1}^{\prime}+\hat{p}_{2}^{\prime}+\hat{p}_{3}^{\prime} \in 2 \mathbf{Z}, \\
& -\left(\hat{p}_{2}^{\prime}-\hat{p}_{3}^{\prime}\right) \leq \hat{p}_{2}^{\prime \prime}=\hat{p}_{2}^{\prime}-\hat{p}_{3}^{\prime}-2 i \leq \hat{p}_{2}^{\prime}-\hat{p}_{3}^{\prime}
\end{aligned}
$$

for some $i \in \mathbf{Z}$ with $0 \leq i \leq \hat{p}_{2}^{\prime}-\hat{p}_{3}^{\prime}$. Therefore,

$$
\Lambda^{\prime}=p_{0}^{\prime} y_{0}+p_{1}^{\prime} y_{1}+p_{2}^{\prime} y_{2}+p_{3}^{\prime} y_{3}+p_{4}^{\prime} y_{4}+\varepsilon^{\prime} p_{5}^{\prime} y_{5} \in D\left(K_{2}\right)
$$


with

$$
\begin{aligned}
p_{0}^{\prime} & =-\frac{1}{2} \hat{p}_{0}^{\prime}-3 \hat{p}_{1}^{\prime}=-3 \hat{p}_{1}^{\prime}, \\
p_{1}^{\prime} & =\frac{1}{4} \hat{p}_{0}^{\prime}-\frac{1}{2} \hat{p}_{1}^{\prime}=-\frac{1}{2} \hat{p}_{1}^{\prime}, \\
p_{2}^{\prime} & =\frac{1}{2}\left(\hat{p}_{2}^{\prime}+\hat{p}_{3}^{\prime}+\hat{p}_{4}^{\prime}-\hat{\varepsilon}^{\prime} \hat{p}_{5}^{\prime}\right)=\frac{1}{2}\left(\hat{p}_{2}^{\prime}+\hat{p}_{3}^{\prime}\right), \\
p_{3}^{\prime} & =\frac{1}{2}\left(\hat{p}_{2}^{\prime}+\hat{p}_{3}^{\prime}-\hat{p}_{4}^{\prime}+\hat{\varepsilon}^{\prime} \hat{p}_{5}^{\prime}\right)=\frac{1}{2}\left(\hat{p}_{2}^{\prime}+\hat{p}_{3}^{\prime}\right), \\
p_{4}^{\prime} & =\frac{1}{2}\left(\hat{p}_{2}^{\prime}-\hat{p}_{3}^{\prime}+\hat{p}_{4}^{\prime}+\hat{\varepsilon}^{\prime} \hat{p}_{5}^{\prime}\right)=\frac{1}{2}\left(\hat{p}_{2}^{\prime}-\hat{p}_{3}^{\prime}\right), \\
\varepsilon^{\prime} p_{5}^{\prime} & =\frac{1}{2}\left(\hat{p}_{2}^{\prime}-\hat{p}_{3}^{\prime}-\hat{p}_{4}^{\prime}-\hat{\varepsilon}^{\prime} \hat{p}_{5}^{\prime}\right)=\frac{1}{2}\left(\hat{p}_{2}^{\prime}-\hat{p}_{3}^{\prime}\right) .
\end{aligned}
$$

In particular, $\varepsilon^{\prime}=1, p_{2}^{\prime}=p_{3}^{\prime}=\frac{1}{2}\left(\hat{p}_{2}^{\prime}+\hat{p}_{3}^{\prime}\right), p_{4}^{\prime}=p_{5}^{\prime}=\frac{1}{2}\left(\hat{p}_{2}^{\prime}-\hat{p}_{3}^{\prime}\right)$. Then $p_{0}=p_{0}^{\prime}$ and by the branching laws of $(\operatorname{Spin}(10), \operatorname{Spin}(2) \cdot \operatorname{Spin}(8))$, we get

$$
\begin{aligned}
& p_{1} \geq p_{2}^{\prime} \geq p_{3}, \quad p_{2} \geq p_{3}^{\prime}=p_{2}^{\prime} \geq p_{4}, \\
& p_{3} \geq p_{4}^{\prime} \geq p_{5}, \quad p_{4} \geq p_{5}^{\prime}=p_{4}^{\prime} \geq 0 .
\end{aligned}
$$

Thus $p_{1} \geq p_{2} \geq p_{2}^{\prime}=p_{3}^{\prime} \geq p_{3} \geq p_{4} \geq p_{4}^{\prime}=p_{5}^{\prime} \geq p_{5} \geq 0$. It follows that

$$
\begin{aligned}
& \ell_{1}=p_{1}-\max \left\{p_{2}, p_{2}^{\prime}\right\}=p_{1}-p_{2}, \\
& \ell_{2}=\min \left\{p_{2}, p_{2}^{\prime}\right\}-\max \left\{p_{3}, p_{3}^{\prime}\right\}=p_{2}^{\prime}-p_{3}^{\prime}=0, \\
& \ell_{3}=\min \left\{p_{3}, p_{3}^{\prime}\right\}-\max \left\{p_{4}, p_{4}^{\prime}\right\}=p_{3}-p_{4}, \\
& \ell_{4}=\min \left\{p_{4}, p_{4}^{\prime}\right\}-\max \left\{p_{5}, p_{5}^{\prime}\right\}=p_{4}^{\prime}-p_{5}^{\prime}=0, \\
& \ell_{5}=\min \left\{p_{5}, p_{5}^{\prime}\right\}=p_{5} .
\end{aligned}
$$

Then the coefficient of $X^{p_{1}^{\prime}}=X^{-\frac{1}{2} \hat{p}_{1}^{\prime}}=X^{-\frac{1}{2} \hat{p}_{1}^{\prime \prime}}$ in the (finite) power series expansion in $X$

$$
\begin{aligned}
X^{\varepsilon \varepsilon^{\prime} \ell_{5}} & \prod_{i=1}^{4} \frac{X^{\ell_{i}+1}-X^{-\ell_{i}-1}}{X-X^{-1}} \\
& =X^{\varepsilon \varepsilon^{\prime} p_{5}} \frac{X^{p_{1}-p_{2}+1}-X^{-\left(p_{1}-p_{2}+1\right)}}{X-X^{-1}} \frac{X^{p_{3}-p_{4}+1}-X^{-\left(p_{3}-p_{4}+1\right)}}{X-X^{-1}} \\
& =X^{\varepsilon \varepsilon^{\prime} p_{5}} \sum_{i=0}^{p_{1}-p_{2}} \sum_{j=0}^{p_{3}-p_{4}} X^{\left(p_{1}-p_{2}\right)+\left(p_{3}-p_{4}\right)-2(i+j)}
\end{aligned}
$$

is equal to its multiplicity. Then we have $\Lambda=p_{0} y_{0}+p_{1} y_{1}+p_{2} y_{2}+p_{3} y_{3}+p_{4} y_{4}+\varepsilon p_{5} y_{5} \in$ $D\left(K, K_{0}\right)$ with $p_{0}=p_{0}^{\prime}=-3 \hat{p}_{1}^{\prime}=6 p_{1}^{\prime} \in 3 \mathbf{Z}$.

5.13. Eigenvalue computation. Recall that the standard basis $\mathbf{e}_{\alpha}(\alpha=0,1, \ldots, 5)$ of $\mathfrak{t}=\left\{\left(\theta_{0}, \theta_{1}, \theta_{2}, \theta_{3}, \theta_{4}, \theta_{5}\right) \mid \theta_{\alpha} \in \mathbf{R}\right\}$ corresponds to $2 \sqrt{-1} R\left(2 e_{1}-e_{2}-e_{3}\right) \in \mathfrak{u}(1)$ and $\sqrt{-1} R\left(e_{2}-e_{3}\right), D_{1,4}, D_{1,12}, D_{1,36}, D_{1,57} \in \operatorname{spin}(10)$, respectively. With respect to the inner product $\langle u, v\rangle_{\mathfrak{u}}=-\operatorname{tr} u v$ for $u, v \in \mathfrak{k} \subset \mathfrak{e}_{6} \subset \mathfrak{g l}\left(H_{3}(\mathbf{K}) \mathbf{C}\right)$, 


$$
\left\langle\mathbf{e}_{0}, \mathbf{e}_{0}\right\rangle=72, \quad\left\langle\mathbf{e}_{i}, \mathbf{e}_{i}\right\rangle=6, \quad\left\langle\mathbf{e}_{\alpha}, \mathbf{e}_{\beta}\right\rangle=0
$$

for $1 \leq i \leq 5$ and $0 \leq \alpha \neq \beta \leq 5$. It follows that the inner products of the dual bases $\left\{y_{0}, y_{1}, y_{2}, y_{3}, y_{4}, y_{5}\right\}$ of $\mathfrak{t}^{*}$ corresponding to $\left\{\mathbf{e}_{0}, \mathbf{e}_{1}, \mathbf{e}_{2}, \mathbf{e}_{3}, \mathbf{e}_{4}, \mathbf{e}_{5}\right\}$ of $\mathfrak{t}$ are given by

$$
\begin{aligned}
& \left\langle y_{\alpha}, y_{\beta}\right\rangle=0, \quad(0 \leq \alpha \neq \beta \leq 5), \\
& \left\langle y_{0}, y_{0}\right\rangle=\frac{1}{72}, \quad\left\langle y_{i}, y_{j}\right\rangle=\frac{1}{6}, \quad(1 \leq i \neq j \leq 5) .
\end{aligned}
$$

For

$$
\begin{aligned}
\Lambda & =p_{0} y_{0}+p_{1} y_{1}+p_{2} y_{2}+p_{3} y_{3}+p_{4} y_{4}+\varepsilon p_{5} y_{5} \in D\left(K, K_{0}\right), \\
\Lambda^{\prime} & =p_{0} y_{0}+\frac{p_{0}}{6} y_{1}+p_{2}^{\prime} y_{2}+p_{2}^{\prime} y_{3}+p_{4}^{\prime} y_{4}+p_{4}^{\prime} y_{5} \\
& =-\frac{p_{0}}{3} \hat{y}_{1}+\left(p_{2}^{\prime}+p_{4}^{\prime}\right) \hat{y}_{2}+\left(p_{2}^{\prime}-p_{4}^{\prime}\right) \hat{y}_{3} \in D\left(K_{2}, K_{0}\right), \\
\Lambda^{\prime \prime} & =-\frac{p_{0}}{3} \hat{y}_{1}+\hat{p}_{2}^{\prime \prime} \hat{y}_{2} \in D\left(K_{1}, K_{0}\right)
\end{aligned}
$$

the eigenvalue formulas of the Casimir operators $\mathcal{C}_{K / K_{0}}, \mathcal{C}_{K_{2} / K_{0}}$ and $\mathcal{C}_{K_{1} / K_{0}}$ with respect to the inner product $\langle,\rangle_{\mathfrak{u}}$ are given respectively by

$$
\begin{aligned}
-c_{\Lambda} & =\frac{1}{72} p_{0}^{2}+\frac{1}{6}\left\{\left(p_{1}+8\right) p_{1}+\left(p_{2}+6\right) p_{2}+\left(p_{3}+4\right) p_{3}+\left(p_{4}+2\right) p_{4}+\left(p_{5}\right)^{2}\right\}, \\
-c_{\Lambda^{\prime}} & =\frac{1}{72}\left(p_{0}^{\prime}\right)^{2}+\frac{1}{6}\left\{\left(p_{1}^{\prime}\right)^{2}+\left(p_{2}^{\prime}+6\right) p_{2}^{\prime}+\left(p_{3}^{\prime}+4\right) p_{3}^{\prime}+\left(p_{4}^{\prime}+2\right) p_{4}^{\prime}+\left(p_{5}^{\prime}\right)^{2}\right\} \\
& =\frac{1}{72}\left(\hat{p}_{0}^{\prime}\right)^{2}+\frac{1}{6}\left\{\left(\hat{p}_{1}^{\prime}\right)^{2}+\left(\hat{p}_{2}^{\prime}+6\right) \hat{p}_{2}^{\prime}+\left(\hat{p}_{3}^{\prime}+4\right) \hat{p}_{3}^{\prime}+\left(\hat{p}_{4}^{\prime}+2\right) \hat{p}_{4}^{\prime}+\left(\hat{p}_{5}^{\prime}\right)^{2}\right\} \\
& =\frac{1}{72}\left(p_{0}\right)^{2}+\frac{1}{6}\left\{\left(\frac{1}{6} p_{0}\right)^{2}+\left(p_{2}^{\prime}+6\right) p_{2}^{\prime}+\left(p_{2}^{\prime}+4\right) p_{2}^{\prime}+\left(p_{4}^{\prime}+2\right) p_{4}^{\prime}+\left(p_{4}^{\prime}\right)^{2}\right\}, \\
-c_{\Lambda^{\prime \prime}} & =\frac{1}{72}\left(\hat{p}_{0}^{\prime \prime}\right)^{2}+\frac{1}{6}\left\{\left(\hat{p}_{1}^{\prime \prime}\right)^{2}+\left(\hat{p}_{2}^{\prime \prime}\right)^{2}+\left(\hat{p}_{3}^{\prime \prime}+4\right) \hat{p}_{3}^{\prime \prime}+\left(\hat{p}_{4}^{\prime \prime}+2\right) \hat{p}_{4}^{\prime \prime}+\left(\hat{p}_{5}^{\prime \prime}\right)^{2}\right\} \\
& =\frac{1}{6}\left\{\left(\frac{1}{3} p_{0}\right)^{2}+\left(\hat{p}_{2}^{\prime \prime}\right)^{2}\right\} .
\end{aligned}
$$


Then for each $\Lambda \in D\left(K, K_{0}\right)$, we have the following eigenvalue formulas

$$
\begin{aligned}
-c_{L}= & -12 c_{\Lambda}+6 c_{\Lambda^{\prime}}+3 c_{\Lambda^{\prime \prime}} \\
= & 2\left\{\left(p_{1}+8\right) p_{1}+\left(p_{2}+6\right) p_{2}+\left(p_{3}+4\right) p_{3}+\left(p_{4}+2\right) p_{4}+\left(p_{5}\right)^{2}\right\} \\
& -\left\{\left(p_{2}^{\prime}+6\right) p_{2}^{\prime}+\left(p_{2}^{\prime}+4\right) p_{2}^{\prime}+\left(p_{4}^{\prime}+2\right) p_{4}^{\prime}+\left(p_{4}^{\prime}\right)^{2}\right\}-\frac{1}{2}\left(\hat{p}_{2}^{\prime \prime}\right)^{2} \\
= & 2\left(p_{1}+8\right) p_{1}+2\left(\left(p_{2}\right)^{2}-\left(p_{2}^{\prime}\right)^{2}\right)+12 p_{2}-10 p_{2}^{\prime}+2\left(p_{3}\right)^{2}+8 p_{3} \\
& +2\left(\left(p_{4}\right)^{2}-\left(p_{4}^{\prime}\right)^{2}\right)+4 p_{4}-2 p_{4}^{\prime}+2\left(p_{5}\right)^{2}-\frac{1}{2}\left(\hat{p}_{2}^{\prime \prime}\right)^{2} \\
= & 2\left(p_{1}+8\right) p_{1}+2\left(\left(p_{2}\right)^{2}-\left(p_{2}^{\prime}\right)^{2}\right)+2 p_{2}+10\left(p_{2}-p_{2}^{\prime}\right)+2\left(p_{3}\right)^{2}+8 p_{3} \\
& +2\left(\left(p_{4}\right)^{2}-\left(p_{4}^{\prime}\right)^{2}\right)+2 p_{4}+2\left(p_{4}-p_{4}^{\prime}\right)+2\left(p_{5}\right)^{2}-\frac{1}{2}\left(\hat{p}_{2}^{\prime \prime}\right)^{2} \\
\geq & 2\left(p_{1}+8\right) p_{1}+2 p_{2}+2\left(p_{3}\right)^{2}-\frac{1}{2}\left(\hat{p}_{2}^{\prime \prime}\right)^{2}+8 p_{3}+2 p_{4}+2\left(p_{5}\right)^{2} \\
= & 2\left(p_{1}+8\right) p_{1}+2 p_{2}+\left(2\left(p_{5}^{\prime}\right)^{2}-\frac{1}{2}\left(\hat{p}_{2}^{\prime \prime}\right)^{2}\right)+8 p_{3}+2 p_{4}+2\left(p_{5}\right)^{2} \\
\geq & 2\left(p_{1}+8\right) p_{1}+2 p_{2}+8 p_{3}+2 p_{4}+2\left(p_{5}\right)^{2},
\end{aligned}
$$

where the equalities hold if and only if $p_{2}=p_{2}^{\prime}, p_{4}=p_{4}^{\prime}, 2 p_{3}=2 p_{4}=2 p_{4}^{\prime}=2 p_{5}^{\prime}=\left|\hat{p}_{2}^{\prime \prime}\right|$ since we have

$$
\begin{aligned}
& p_{1} \geq p_{2} \geq p_{2}^{\prime}=p_{3}^{\prime} \geq p_{3} \geq p_{4} \geq p_{4}^{\prime}=p_{5}^{\prime} \geq p_{5} \geq 0, \\
& -2 p_{4}^{\prime}=-2 p_{5}^{\prime}=-\left(\hat{p}_{2}^{\prime}-\hat{p}_{3}^{\prime}\right) \leq \hat{p}_{2}^{\prime \prime} \leq \hat{p}_{2}^{\prime}-\hat{p}_{3}^{\prime}=2 p_{5}^{\prime}=2 p_{4}^{\prime} .
\end{aligned}
$$

Notice that if $p_{1}=0$, then $-c_{L}=0$ and if $p_{1} \geq 2$, then $-c_{L} \geq 40>30$. In case $p_{1}=3 / 2$, the possible $\Lambda=\left(p_{0}, p_{1}, p_{2}, p_{3}, p_{4}, p_{5}\right) \in D\left(K, K_{0}\right)$ are

$$
\begin{aligned}
& \left(p_{0}, \frac{3}{2}, \frac{3}{2}, \frac{3}{2}, \frac{3}{2}, \frac{3}{2}\right),\left(p_{0}, \frac{3}{2}, \frac{3}{2}, \frac{3}{2}, \frac{3}{2},-\frac{3}{2}\right),\left(p_{0}, \frac{3}{2}, \frac{3}{2}, \frac{3}{2}, \frac{3}{2}, \frac{1}{2}\right),\left(p_{0}, \frac{3}{2}, \frac{3}{2}, \frac{3}{2}, \frac{3}{2},-\frac{1}{2}\right), \\
& \left(p_{0}, \frac{3}{2}, \frac{3}{2}, \frac{3}{2}, \frac{1}{2}, \frac{1}{2}\right),\left(p_{0}, \frac{3}{2}, \frac{3}{2}, \frac{3}{2}, \frac{1}{2},-\frac{1}{2}\right),\left(p_{0}, \frac{3}{2}, \frac{3}{2}, \frac{1}{2}, \frac{1}{2}, \frac{1}{2}\right),\left(p_{0}, \frac{3}{2}, \frac{3}{2}, \frac{1}{2}, \frac{1}{2},-\frac{1}{2}\right), \\
& \left(p_{0}, \frac{3}{2}, \frac{1}{2}, \frac{1}{2}, \frac{1}{2}, \frac{1}{2}\right),\left(p_{0}, \frac{3}{2}, \frac{1}{2}, \frac{1}{2}, \frac{1}{2},-\frac{1}{2}\right) .
\end{aligned}
$$

In these cases, the eigenvalue of the Casimir operator $\mathcal{C}_{L}$ is given by

$$
\begin{aligned}
-c_{L} & \geq 2\left(p_{1}+8\right) p_{1}+2 p_{2}+8 p_{3}+2 p_{4}+2\left(p_{5}\right)^{2} \\
& \geq 2 \cdot\left(\frac{3}{2}+8\right) \cdot \frac{3}{2}+2 \cdot \frac{1}{2}+8 \cdot \frac{1}{2}+2 \cdot \frac{1}{2}+2 \cdot\left(\frac{1}{2}\right)^{2} \\
& =35>30 .
\end{aligned}
$$

Hence in order to determine the Hamiltonian stability, i.e., to compare the first eigenvalue $-c_{L}$ and 30 , we have only to treat the cases when $p_{1}=1 / 2$ or 1 . 
TABLE 5. Small eigenvalues of $-\mathcal{C}_{L}$ for $L=U(1) \cdot \operatorname{Spin}(10) /\left(S^{1} \cdot \operatorname{Spin}(6) \cdot \mathbf{Z}_{4}\right)$.

\begin{tabular}{|l|l|l|l|}
\hline$\Lambda$ & $\Lambda^{\prime}$ & $\Lambda^{\prime \prime}$ & $-c_{L}$ \\
\hline $3, \frac{1}{2}, \frac{1}{2}, \frac{1}{2}, \frac{1}{2}, \frac{1}{2}$ & $3, \frac{1}{2}, \frac{1}{2}, \frac{1}{2}, \frac{1}{2}, \frac{1}{2}$ & $0,-1,1,0,0,0$ & 15 \\
\hline $3, \frac{1}{2}, \frac{1}{2}, \frac{1}{2}, \frac{1}{2}, \frac{1}{2}$ & $3, \frac{1}{2}, \frac{1}{2}, \frac{1}{2}, \frac{1}{2}, \frac{1}{2}$ & $0,-1,-1,0,0,0$ & 15 \\
\hline$-3, \frac{1}{2}, \frac{1}{2}, \frac{1}{2}, \frac{1}{2},-\frac{1}{2}$ & $-3,-\frac{1}{2}, \frac{1}{2}, \frac{1}{2}, \frac{1}{2}, \frac{1}{2}$ & $0,1,1,0,0,0$ & 15 \\
\hline$-3, \frac{1}{2}, \frac{1}{2}, \frac{1}{2}, \frac{1}{2},-\frac{1}{2}$ & $-3,-\frac{1}{2}, \frac{1}{2}, \frac{1}{2}, \frac{1}{2}, \frac{1}{2}$ & $0,1,-1,0,0,0$ & 15 \\
\hline $6,1,0,0,0,0$ & $6,1,0,0,0,0$ & $0,-2,0,0,0,0$ & 18 \\
\hline$-6,1,0,0,0,0$ & $-6,-1,0,0,0,0$ & $0,2,0,0,0,0$ & 18 \\
\hline $0,1,1,0,0,0$ & $0,0,0,0,0,0$ & $0,0,0,0,0,0$ & 32 \\
\hline $0,1,1,0,0,0$ & $0,0,1,1,0,0$ & $0,0,0,0,0,0$ & 20 \\
\hline $6,1,1,1,0,0$ & $6,1,1,1,0,0$ & $0,-2,0,0,0,0$ & 30 \\
\hline$-6,1,1,1,0,0$ & $-6,-1,1,1,0,0$ & $0,2,0,0,0,0$ & 30 \\
\hline $0,1,1,1,1,0$ & $0,0,1,1,0,0$ & $0,0,0,0,0,0$ & 36 \\
\hline $0,1,1,1,1,0$ & $0,0,1,1,1,1$ & $0,0,0,0,0,0$ & 32 \\
\hline $0,1,1,1,1,0$ & $0,0,1,1,1,1$ & $0,0,2,0,0,0$ & 30 \\
\hline $0,1,1,1,1,0$ & $0,0,1,1,1,1$ & $0,0,-2,0,0,0$ & 30 \\
\hline $6,1,1,1,1,1$ & $6,1,1,1,1,1$ & $0,-2,2,0,0,0$ & 32 \\
\hline $6,1,1,1,1,1$ & $6,1,1,1,1,1$ & $0,-2,-2,0,0,0$ & 32 \\
\hline $6,1,1,1,1,1$ & $6,1,1,1,1,1$ & $0,-2,0,0,0,0$ & 34 \\
\hline$-6,1,1,1,1,-1$ & $-6,-1,1,1,1,1$ & $0,2,2,0,0,0$ & 32 \\
\hline$-6,1,1,1,1,-1$ & $-6,-1,1,1,1,1$ & $0,2,-2,0,0,0$ & 32 \\
\hline$-6,1,1,1,1,-1$ & $-6,-1,1,1,1,1$ & $0,2,0,0,0,0$ & 34 \\
\hline
\end{tabular}

It follows from the description of $D\left(K, K_{0}\right)$ in Section 5.12 that the element in $D\left(K, K_{0}\right)$. when $p_{1}=1 / 2$ is given by

$$
\left(p_{0}, \frac{1}{2}, \frac{1}{2}, \frac{1}{2}, \frac{1}{2}, \frac{1}{2}\right) \text { or }\left(p_{0}, \frac{1}{2}, \frac{1}{2}, \frac{1}{2}, \frac{1}{2},-\frac{1}{2}\right)
$$

and the element in $D\left(K, K_{0}\right)$ for $p_{1}=1$ is given by

$$
\begin{array}{ll}
\left(p_{0}, 1,0,0,0,0\right), & \left(p_{0}, 1,1,0,0,0\right), \quad\left(p_{0}, 1,1,1,0,0\right), \\
\left(p_{0}, 1,1,1,1,0\right), & \left(p_{0}, 1,1,1,1,1\right) \text { or }\left(p_{0}, 1,1,1,1,-1\right) .
\end{array}
$$

Using the branching laws, the descriptions of $D\left(K_{2}, K_{0}\right), D\left(K_{1}, K_{0}\right)$ in Section 5.12 and the eigenvalue formula (44), by direct computation we get the following small eigenvalues in Table 5. Here, $\Lambda=\left(p_{0}, p_{1}, p_{2}, p_{3}, p_{4}, p_{5}\right) \in D\left(K, K_{0}\right), \Lambda^{\prime}=\left(p_{0}^{\prime}, p_{1}^{\prime}, p_{2}^{\prime}, p_{3}^{\prime}, p_{4}^{\prime}, p_{5}^{\prime}\right) \in$ $D\left(K_{2}, K_{0}\right)$ and $\Lambda^{\prime \prime}=\left(\hat{p}_{0}^{\prime \prime}, \hat{p}_{1}^{\prime \prime}, \hat{p}_{2}^{\prime \prime}, \hat{p}_{3}^{\prime \prime}, \hat{p}_{4}^{\prime \prime}, \hat{p}_{5}^{\prime \prime}\right) \in D\left(K_{1}, K_{0}\right)$. The next lemma follows from Table 5. 
LEMMA 5.5. $\Lambda=p_{0} y_{0}+p_{1} y_{1}+p_{2} y_{2}+p_{3} y_{3}+p_{4} y_{4}+\varepsilon p_{5} y_{5} \in D\left(K, K_{0}\right)$ has eigenvalue $-c_{L} \leq 30$ if and only if $\left(p_{0}, p_{1}, p_{2}, p_{3}, p_{4}, p_{5}\right)$ is one of

$$
\left\{0,\left(3, \frac{1}{2}, \frac{1}{2}, \frac{1}{2}, \frac{1}{2}, \frac{1}{2}\right),\left(-3, \frac{1}{2}, \frac{1}{2}, \frac{1}{2}, \frac{1}{2},-\frac{1}{2}\right),(6,1,0,0,0,0),(-6,1,0,0,0,0)\right.
$$

$(0,1,1,0,0,0),(6,1,1,1,0,0),(-6,1,1,1,0,0),(0,1,1,1,1,0)\}$.

Since $\Lambda_{1}=\left(3, \frac{1}{2}, \frac{1}{2}, \frac{1}{2}, \frac{1}{2}, \frac{1}{2}\right)$ corresponds to the complexified isotropy representation of EIII and it is conjugate to $\Lambda_{2}=\left(-3, \frac{1}{2}, \frac{1}{2}, \frac{1}{2}, \frac{1}{2},-\frac{1}{2}\right)$, we see that $\Lambda_{1}, \Lambda_{2} \notin D\left(K, K_{[\mathfrak{a}]}\right)$.

Suppose that $\Lambda=\left(p_{0}, p_{1}, p_{2}, p_{3}, p_{4}, p_{5}\right)=(6,1,0,0,0,0) \in D\left(K, K_{0}\right)$. Then by the branching laws we get $\Lambda^{\prime}=6 y_{0}+y_{1} \in D\left(K_{2}, K_{0}\right), \Lambda^{\prime \prime}=-2 \hat{y}_{1} \in D\left(K_{1}, K_{0}\right)$ and $\Lambda^{\prime \prime \prime}=0 \in D\left(K_{0}\right)$. Hence, the eigenvalue of the Casimir operator is $-c_{L}=18<30$.

On the other hand,

$$
\begin{aligned}
V_{\Lambda} \cong & \left\{\left(\begin{array}{ccc}
0 & 0 & 0 \\
0 & \xi_{2} & x_{1} \\
0 & \bar{x}_{1} & \xi_{3}
\end{array}\right) \mid \xi_{2}, \xi_{3} \in \mathbf{C}, x_{1} \in \mathbf{K}^{\mathbf{C}}\right\} \cong \mathbf{C}^{10} \\
& \supset W_{\Lambda^{\prime}}=U_{\Lambda^{\prime \prime}}=U_{\Lambda^{\prime \prime \prime}}=\left(V_{\Lambda}\right)_{K_{0}}
\end{aligned}
$$

and $\rho_{\Lambda}=\mu_{6} \otimes \sigma_{\mathbf{C}^{10}}$, where $\sigma_{\mathbf{C}^{10}}$ denotes the standard representation of $S O(10)$, and for each $\phi(\theta) \in U(1)$

$$
\mu_{6}(\phi(\theta))\left(\begin{array}{ccc}
0 & 0 & 0 \\
0 & \xi_{2} & x_{1} \\
0 & \bar{x}_{1} & \xi_{3}
\end{array}\right)=\theta^{-6}\left(\begin{array}{ccc}
0 & 0 & 0 \\
0 & \xi_{2} & x_{1} \\
0 & \bar{x}_{1} & \xi_{3}
\end{array}\right)
$$

where $\theta=e^{\sqrt{-1} t_{0} / 2}$. Since for any $\exp \left(\hat{t}_{0} \sqrt{-1} R\left(e_{1}-2 e_{2}+e_{3}\right)\right) \in S^{1} \subset K_{0}$,

$$
\begin{aligned}
\exp \left(\hat{t}_{0} \sqrt{-1}\right. & \left.R\left(e_{1}-2 e_{2}+e_{3}\right)\right) \\
& =\exp \left(\hat{t}_{0} \frac{1}{2} \sqrt{-1} R\left(2 e_{1}-e_{2}-e_{3}\right)\right) \exp \left(-\hat{t}_{0} \frac{3}{2} \sqrt{-1} R\left(e_{2}-e_{3}\right)\right) \\
& \in U(1) \cdot \operatorname{Spin}(2) \subset K,
\end{aligned}
$$


we compute

$$
\begin{aligned}
& \rho_{\Lambda}\left(\exp \left(\hat{t}_{0} \sqrt{-1} R\left(e_{1}-2 e_{2}+e_{3}\right)\right)\right)\left(\begin{array}{ccc}
0 & 0 & 0 \\
0 & \xi_{2} & x_{1} \\
0 & \bar{x}_{1} & \xi_{3}
\end{array}\right) \\
& =\left(\mu_{6} \otimes \sigma_{\mathbf{C}^{10}}\right)\left(\exp \left(\hat{t}_{0} \sqrt{-1} R\left(e_{1}-2 e_{2}+e_{3}\right)\right)\right)\left(\begin{array}{ccc}
0 & 0 & 0 \\
0 & \xi_{2} & x_{1} \\
0 & \bar{x}_{1} & \xi_{3}
\end{array}\right) \\
& =\mu_{6}\left(\exp \left(\hat{t}_{0} \frac{1}{2} \sqrt{-1} R\left(2 e_{1}-e_{2}-e_{3}\right)\right)\right) \alpha_{23}\left(-\hat{t}_{0} \frac{3}{2}\right)\left(\begin{array}{ccc}
0 & 0 & 0 \\
0 & \xi_{2} & x_{1} \\
0 & \bar{x}_{1} & \xi_{3}
\end{array}\right) \\
& =\left(e^{\sqrt{-1} \frac{1}{2} \hat{t}_{0} \frac{1}{2}}\right)^{-6}\left(\begin{array}{ccc}
0 & 0 & 0 \\
0 & e^{-\sqrt{-1} \hat{t}_{0} \frac{3}{2} \xi_{2}} & x_{1} \\
0 & \bar{x}_{1} & e^{\sqrt{-1} \hat{t}_{0} \frac{3}{2} \xi_{3}}
\end{array}\right) \\
& =e^{-\sqrt{-1} \frac{3}{2} \hat{t}_{0}}\left(\begin{array}{ccc}
0 & 0 & 0 \\
0 & e^{-\sqrt{-1} \hat{t}_{0} \frac{3}{2} \xi_{2}} & x_{1} \\
0 & \bar{x}_{1} & e^{\sqrt{-1} \hat{t}_{0} \frac{3}{2} \xi_{3}}
\end{array}\right) \\
& =\left(\begin{array}{ccc}
0 & 0 & 0 \\
0 & e^{-\sqrt{-1} 3 \hat{t}_{0}} \xi_{2} & e^{-\sqrt{-1} \frac{3}{2} \hat{t}_{0}} x_{1} \\
0 & e^{-\sqrt{-1} \frac{3}{2} \hat{t}_{0} \bar{x}_{1}} & \xi_{3}
\end{array}\right) \text {. }
\end{aligned}
$$

In particular,

$$
\rho_{\Lambda}\left(\exp \left(\hat{t}_{0} \sqrt{-1} R\left(e_{1}-2 e_{2}+e_{3}\right)\right)\right)\left(\begin{array}{ccc}
0 & 0 & 0 \\
0 & 0 & 0 \\
0 & 0 & \xi_{3}
\end{array}\right)=\left(\begin{array}{ccc}
0 & 0 & 0 \\
0 & 0 & 0 \\
0 & 0 & \xi_{3}
\end{array}\right)
$$

for each $\hat{t}_{0} \in \mathbf{R}$. Hence,

$$
\left(V_{\Lambda}\right)_{K_{0}} \cong\left\{\left(\begin{array}{ccc}
0 & 0 & 0 \\
0 & 0 & 0 \\
0 & 0 & \xi_{3}
\end{array}\right) \mid \xi_{3} \in \mathbf{C}\right\}
$$

But as a generator of $\mathbf{Z}_{4}$ of $K_{[\mathfrak{a}]}$, the action of $\alpha_{23}(\pi)\left(\alpha_{1}, \alpha_{2}, \alpha_{3}\right) \in K_{[\mathfrak{a}]}$ given by (42) is

$$
\begin{aligned}
& \rho_{\Lambda}\left(\alpha_{23}(\pi)\left(\alpha_{1}, \alpha_{2}, \alpha_{3}\right)\right)\left(\begin{array}{ccc}
0 & 0 & 0 \\
0 & 0 & 0 \\
0 & 0 & \xi_{3}
\end{array}\right) \\
= & \left(\alpha_{23}(\pi)\right)\left(\begin{array}{ccc}
0 & 0 & 0 \\
0 & 0 & 0 \\
0 & 0 & \xi_{3}
\end{array}\right)=\left(\begin{array}{ccc}
0 & 0 & 0 \\
0 & 0 & 0 \\
0 & 0 & -\xi_{3}
\end{array}\right) .
\end{aligned}
$$

Therefore $\left(V_{\Lambda}\right)_{K_{[\mathfrak{a}]}}=\{0\}$ and $\Lambda=6 y_{0}+y_{1} \notin D\left(K, K_{[\mathfrak{a}]}\right)$. Similarly, $\Lambda=-6 y_{0}+y_{1} \notin$ $D\left(K, K_{[\mathfrak{a}]}\right)$. 
Suppose $\Lambda=\left(p_{0}, p_{1}, p_{2}, p_{3}, p_{4}, p_{5}\right)=(0,1,1,0,0,0) \in D\left(K, K_{0}\right)$. Then by the branching laws we get

$$
\begin{aligned}
& \Lambda^{\prime}=\left(p_{0}^{\prime}, p_{1}^{\prime}, p_{2}^{\prime}, p_{3}^{\prime}, p_{4}^{\prime}, p_{5}^{\prime}\right)=(0,0,1,1,0,0) \in D\left(K_{2}, K_{0}\right), \\
& \Lambda^{\prime \prime}=\left(\hat{p}_{0}^{\prime \prime}, \hat{p}_{1}^{\prime \prime}, \hat{p}_{2}^{\prime \prime}, \hat{p}_{3}^{\prime \prime}, \hat{p}_{4}^{\prime \prime}, \hat{p}_{5}^{\prime \prime}\right)=(0,0,0,0,0,0) \in D\left(K_{1}, K_{0}\right) .
\end{aligned}
$$

Here $\rho_{\Lambda^{\prime}}^{\prime}=\operatorname{Id} \otimes \operatorname{Id} \otimes \operatorname{Ad}_{S p i n(8)}^{\mathbf{C}}=\operatorname{Id} \otimes \operatorname{Id} \otimes \operatorname{Ad}_{S O(8)}^{\mathbf{C}} \in \mathcal{D}\left(K_{2}\right)$. Notice that $W_{\Lambda^{\prime}}=\mathfrak{o}(8){ }^{\mathbf{C}}=$ $\mathfrak{o}(2)^{\mathbf{C}} \oplus \mathfrak{o}(6)^{\mathbf{C}} \oplus M(2,6 ; \mathbf{R})^{\mathbf{C}}$, and the subgroups $U(1)$ and $\operatorname{Spin}(2)$ of $K_{2}=(U(1) \times$ $(\operatorname{Spin}(2) \cdot \operatorname{Spin}(8)) / \mathbf{Z}_{4}$ act trivially on $\mathfrak{o}(8) \mathbf{C}$. The subgroup $\operatorname{Spin}(6)$ of $\operatorname{Spin}(2) \cdot \operatorname{Spin}(6)$ acts trivially on $\mathfrak{o}(2)^{\mathbf{C}}$, hence $\left(W_{\Lambda^{\prime}}\right)_{K_{0}}=\mathfrak{o}(2)^{\mathbf{C}}$. For $\alpha_{23}(\pi)\left(\alpha_{1}, \alpha_{2}, \alpha_{3}\right) \in K_{[\mathfrak{a}]}$ a generator of $\mathbf{Z}_{4}$ given in (42), $\alpha_{23}(\pi)$ and $\left(\alpha_{1}, \alpha_{2}, \alpha_{3}\right)$ commute to each other. $\alpha_{23}(\pi) \in \operatorname{Spin}(2)$ acts trivially on $\mathfrak{o}(2){ }^{\mathbf{C}} . \alpha_{2}$ of $\left(\alpha_{1}, \alpha_{2}, \alpha_{3}\right)$ acts on $\mathbf{R} 1+\mathbf{R e}$ as $\left(\begin{array}{cc}0 & -1 \\ -1 & 0\end{array}\right)$ and preserves the vector subspace orthogonally complementary to $\mathbf{R} 1+\mathbf{R e}$ in $\mathbf{K} \cong \mathbf{R}^{8}$. Thus the $\operatorname{Spin}(2)$-factor of $\left(\alpha_{1}, \alpha_{2}, \alpha_{3}\right)$ in $\operatorname{Spin}(2) \cdot \operatorname{Spin}(6)$ corresponds to $\left(\begin{array}{cc}0 & -1 \\ -1 & 0\end{array}\right) \in O(2)$. Since its adjoint action on $\mathfrak{o}(2){ }^{\mathbf{C}}$ is $-\mathrm{Id}$, the adjoint action of $\left(\alpha_{1}, \alpha_{2}, \alpha_{3}\right) \in \operatorname{Spin}(8)$ is not trivial on $\mathfrak{o}(2)^{\mathbf{C}}$. Hence $\left(W_{\Lambda^{\prime}}\right)_{K_{[\mathfrak{a}]}}=\{0\}$ and in particular we obtain $\Lambda=y_{1}+y_{2} \notin D\left(K, K_{[\mathfrak{a}]}\right)$.

Suppose $\Lambda=\left(p_{0}, p_{1}, p_{2}, p_{3}, p_{4}, p_{5}\right)=(6,1,1,1,0,0) \in D\left(K, K_{0}\right)$. Then $\operatorname{dim}_{\mathbf{C}} V_{\Lambda}=$ 120. By the branching laws we get $\Lambda^{\prime}=6 y_{0}+y_{1}+y_{2}+y_{3}=-2 \hat{y}_{1}+\hat{y}_{2}+\hat{y}_{3} \in D\left(K_{2}, K_{0}\right)$, $\Lambda^{\prime \prime}=-2 \hat{y}_{1} \in D\left(K_{1}, K_{0}\right)$ and $\Lambda^{\prime \prime \prime}=0 \in D\left(K_{0}\right)$. Hence, the eigenvalue of the Casimir operator is $-c_{L}=30$.

On the other hand, $\rho_{\Lambda^{\prime}}^{\prime}=\operatorname{Id} \otimes \mu_{-2} \otimes \operatorname{Ad}_{S p i n(8)}^{\mathrm{C}}=\operatorname{Id} \otimes \mu_{-2} \otimes \operatorname{Ad}_{S O(8)}^{\mathrm{C}} \in \mathcal{D}\left(K_{2}\right)$. Here $W_{\Lambda^{\prime}}=\mathfrak{o}(8) \mathbf{C}=\mathfrak{o}(2)^{\mathbf{C}} \oplus \mathfrak{o}(6)^{\mathbf{C}} \oplus M(2,6 ; \mathbf{R})^{\mathbf{C}}$. Same as the previous case, we get $\left(W_{\Lambda^{\prime}}\right)_{K_{0}}=\mathfrak{o}(2)^{\mathbf{C}}$. Notice that for the generator $\alpha_{23}(\pi)\left(\alpha_{1}, \alpha_{2}, \alpha_{3}\right)$ of $\mathbf{Z}_{4}$ in $K_{\text {[a] }}$ given by (42), the action of $\alpha_{23}(\pi) \in \operatorname{Spin}(2)$ on $H_{3}\left(\mathbf{K}^{\mathbf{C}}\right)$ is given by

$$
\left(\begin{array}{lll}
\xi_{1} & x_{3} & \bar{x}_{2} \\
\bar{x}_{3} & \xi_{2} & x_{1} \\
x_{2} & \bar{x}_{1} & \xi_{3}
\end{array}\right) \mapsto\left(\begin{array}{ccc}
\xi_{1} & \sqrt{-1} x_{3} & -\sqrt{-1} \bar{x}_{2} \\
\sqrt{-1} \bar{x}_{3} & -\xi_{2} & x_{1} \\
-\sqrt{-1} x_{2} & \bar{x}_{1} & -\xi_{3}
\end{array}\right) .
$$

In particular, $\alpha_{23}(\pi)$ transforms $u_{2}$ to $-\sqrt{-1} u_{2}$ and $\mathbf{e} u_{2}$ to $-\sqrt{-1} \mathbf{e} u_{2}$, which says that $\alpha_{23}(\pi)$ acts on $\mathfrak{o}(2) \cong \mathbf{R} 1+\mathbf{R e}$ as the matrix multiplication by $\left(\begin{array}{cc}-\sqrt{-1} & 0 \\ 0 & -\sqrt{-1}\end{array}\right)$. Thus $\mu_{-2}\left(\alpha_{23}(\pi)\right)$ acts on $\mathfrak{o}(2) \cong \mathbf{R} 1+\mathbf{R e}$ is just the matrix multiplication by - Id. On the other hand, $\alpha_{2}$ of $\left(\alpha_{1}, \alpha_{2}, \alpha_{3}\right)$ acts on $\mathbf{R} 1+\mathbf{R} e$ as $\left(\begin{array}{cc}0 & -1 \\ -1 & 0\end{array}\right)$. Thus the $\operatorname{Spin}(2)$-factor of $\left(\alpha_{1}, \alpha_{2}, \alpha_{3}\right)$ in $\operatorname{Spin}(2)$. $\operatorname{Spin}(6)$ corresponds to $\left(\begin{array}{cc}0 & -1 \\ -1 & 0\end{array}\right) \in O(2)$. Hence its adjoint action on $\mathfrak{o}(2) \mathbf{C}$ is - Id. Therefore, $\left(V_{\Lambda}\right)_{K_{[\mathfrak{a}]}}=\mathfrak{o}(2)^{\mathbf{C}}$, i.e., $\Lambda=6 y_{0}+y_{1}+y_{2}+y_{3} \in D\left(K, K_{[\mathfrak{a}]}\right)=\mathfrak{o}(2){ }^{\mathbf{C}}$. Thus $\Lambda=6 y_{0}+y_{1}+$ $y_{2}+y_{3} \in D\left(K, K_{[\mathfrak{a}]}\right)$ with multiplicity 1 . Similarly, $\Lambda=-6 y_{0}+y_{1}+y_{2}+y_{3} \in D\left(K, K_{[\mathfrak{a}]}\right)$ with multiplicity 1 . 
Suppose $\Lambda=\left(p_{0}, p_{1}, p_{2}, p_{3}, p_{4}, p_{5}\right)=(0,1,1,1,1,0) \in D\left(K, K_{0}\right)$. Then $\operatorname{dim}_{\mathbf{C}} V_{\Lambda}=$ 210. By Subsection 5.12, we describe explicitly $\left(V_{\Lambda}\right)_{K_{0}}$ as follows:

$$
\begin{aligned}
& \left(V_{\Lambda(0,1,1,1,1,0)}\right)_{K_{0}} \\
& \quad=W_{\Lambda_{1}^{\prime}(0,0,1,1,1,1)} \cap\left(V_{\Lambda}\right)_{K_{0}} \oplus W_{\Lambda_{2}^{\prime}(0,0,1,1,0,0)} \cap\left(V_{\Lambda}\right)_{K_{0}} \\
& \quad=\left(U_{\Lambda_{1}^{\prime \prime}(0,0,0,0,0,0)} \oplus U_{\Lambda_{1}^{\prime \prime}(0,0,2,0,0,0)} \oplus U_{\Lambda_{1}^{\prime \prime}(0,0,-2,0,0,0)}\right)_{K_{0}} \oplus U_{\Lambda_{2}^{\prime \prime}(0,0,0,0,0,0)} .
\end{aligned}
$$

Then the Casimir operator $-\mathcal{C}_{L}$ has eigenvalues $-c_{L}=32,30,30$ or 36 along this decomposition.

On the other hand, $\Lambda_{1}^{\prime}=2 \hat{y}_{2} \in D\left(K_{2}, K_{0}\right), W_{\Lambda_{1}^{\prime}} \cong S_{0}^{2}\left(\mathbf{C}^{8}\right) \cong S_{0}^{2}\left(\mathbf{K}^{8}\right)$ and

$$
W_{\Lambda_{1}^{\prime}} \cap\left(V_{\Lambda}\right)_{K_{0}}=U_{\Lambda_{1}^{\prime \prime}(0,0,0,0,0,0)} \oplus\left(U_{\Lambda_{1}^{\prime \prime}(0,0,2,0,0,0)}\right)_{K_{0}} \oplus\left(U_{\Lambda_{1}^{\prime \prime}(0,0,-2,0,0,0)}\right)_{K_{0}} .
$$

Recall that $\left\{1, c_{1}, \ldots, c_{7}\right\}$ denote the standard basis of the Cayley algebra $\mathbf{K}$ and $\mathbf{e}:=c_{4}$. Then

$$
3(1 \cdot 1+\mathbf{e} \cdot \mathbf{e})-\left(c_{1} \cdot c_{1}+c_{2} \cdot c_{2}+c_{3} \cdot c_{3}+c_{5} \cdot c_{5}+c_{6} \cdot c_{6}+c_{7} \cdot c_{7}\right) \in S_{0}^{2}\left(\mathbf{K}^{\mathbf{C}}\right) .
$$

For any $A=\left(\begin{array}{c}\cos t-\sin t \\ \sin t \cos t\end{array}\right) \in S O(2), A(1, \mathbf{e})=(1, \mathbf{e})\left(\begin{array}{c}\cos t-\sin t \\ \sin t \cos t\end{array}\right)$. Hence

$$
\begin{aligned}
A(1 \cdot 1) & =(\cos t 1+\sin t \mathbf{e}) \cdot(\cos t 1+\sin t \mathbf{e}) \\
& =\cos ^{2} t(1 \cdot 1)+\sin ^{2} t(\mathbf{e} \cdot \mathbf{e})+2 \sin t \cos t(1 \cdot \mathbf{e}), \\
A(\mathbf{e} \cdot \mathbf{e}) & =(-\sin t 1+\cos t \mathbf{e}) \cdot(-\sin t 1+\cos t \mathbf{e}) \\
& =\sin ^{2} t(1 \cdot 1)+\cos ^{2} t(\mathbf{e} \cdot \mathbf{e})-2 \sin t \cos t(1 \cdot \mathbf{e}), \\
A(1 \cdot \mathbf{e}) & =(\cos t 1+\sin t \mathbf{e}) \cdot(-\sin t 1+\cos t \mathbf{e}) \\
& =-\frac{1}{2} \sin 2 t(1 \cdot 1-\mathbf{e} \cdot \mathbf{e})+\cos 2 t(1 \cdot \mathbf{e}) .
\end{aligned}
$$

In particular, $A(1 \cdot 1+\mathbf{e} \cdot \mathbf{e})=1 \cdot 1+\mathbf{e} \cdot \mathbf{e}$ and

$$
\begin{aligned}
& A\left(3(1 \cdot 1+\mathbf{e} \cdot \mathbf{e})-\left(c_{1} \cdot c_{1}+c_{2} \cdot c_{2}+c_{3} \cdot c_{3}+c_{5} \cdot c_{5}+c_{6} \cdot c_{6}+c_{7} \cdot c_{7}\right)\right) \\
& \quad=3(1 \cdot 1+\mathbf{e} \cdot \mathbf{e})-\left(c_{1} \cdot c_{1}+c_{2} \cdot c_{2}+c_{3} \cdot c_{3}+c_{5} \cdot c_{5}+c_{6} \cdot c_{6}+c_{7} \cdot c_{7}\right) .
\end{aligned}
$$

Thus, $3(1 \cdot 1+\mathbf{e} \cdot \mathbf{e})-\left(c_{1} \cdot c_{1}+c_{2} \cdot c_{2}+c_{3} \cdot c_{3}+c_{5} \cdot c_{5}+c_{6} \cdot c_{6}+c_{7} \cdot c_{7}\right) \in U_{\Lambda^{\prime \prime}(0,0,0,0,0,0)}$. On the other hand, $1 \cdot 1-\mathbf{e} \cdot \mathbf{e}-2 \sqrt{-1}(1 \cdot \mathbf{e}), 1 \cdot 1-\mathbf{e} \cdot \mathbf{e}+2 \sqrt{-1}(1 \cdot \mathbf{e}) \in S_{0}^{2}\left(\mathbf{K}^{\mathbf{C}}\right)$, and we see that

$$
\begin{aligned}
& A(1 \cdot 1-\mathbf{e} \cdot \mathbf{e}-2 \sqrt{-1} 1 \cdot \mathbf{e})=\mathbf{e}^{\sqrt{-1} 2 t}(1 \cdot 1-\mathbf{e} \cdot \mathbf{e}-2 \sqrt{-1} 1 \cdot \mathbf{e}), \\
& A(1 \cdot 1-\mathbf{e} \cdot \mathbf{e}+2 \sqrt{-1} 1 \cdot \mathbf{e})=\mathbf{e}^{-\sqrt{-1} 2 t}(1 \cdot 1-\mathbf{e} \cdot \mathbf{e}+2 \sqrt{-1} 1 \cdot \mathbf{e}) .
\end{aligned}
$$

Hence, $1 \cdot 1-\mathbf{e} \cdot \mathbf{e}-2 \sqrt{-1} 1 \cdot \mathbf{e} \in U_{\Lambda^{\prime \prime}(0,0,2,0,0,0)}, 1 \cdot 1-e \cdot \mathbf{e}+2 \sqrt{-1} 1 \cdot \mathbf{e} \in U_{\Lambda^{\prime \prime}(0,0,-2,0,0,0)}$. Therefore,

$$
\begin{aligned}
\left(V_{\Lambda}\right)_{K_{0}} & \cap W_{\Lambda_{1}^{\prime}} \\
= & \mathbf{C}\left(3(1 \cdot 1+\mathbf{e} \cdot \mathbf{e})-\left(c_{1} \cdot c_{1}+c_{2} \cdot c_{2}+c_{3} \cdot c_{3}+c_{5} \cdot c_{5}+c_{6} \cdot c_{6}+c_{7} \cdot c_{7}\right)\right) \\
& \oplus \mathbf{C}(1 \cdot 1-\mathbf{e} \cdot \mathbf{e}-2 \sqrt{-1} 1 \cdot \mathbf{e}) \oplus \mathbf{C}(1 \cdot 1-\mathbf{e} \cdot \mathbf{e}+2 \sqrt{-1} 1 \cdot \mathbf{e}) .
\end{aligned}
$$


Since the action of the generator $\alpha_{23}(\pi)\left(\alpha_{1}, \alpha_{2}, \alpha_{3}\right)$ is given by

$$
\begin{gathered}
\left(\alpha_{23}(\pi)\left(\alpha_{1}, \alpha_{2}, \alpha_{3}\right)\right)(2 \sqrt{-1}(1 \cdot \mathbf{e}))=2(\sqrt{-1} \mathbf{e} \cdot(-1))=-2 \sqrt{-1}(1 \cdot \mathbf{e}), \\
\left(\alpha_{23}(\pi)\left(\alpha_{1}, \alpha_{2}, \alpha_{3}\right)\right)(1 \cdot 1-\mathbf{e} \cdot \mathbf{e})=1 \cdot 1-\mathbf{e} \cdot \mathbf{e}, \\
\left(\alpha_{23}(\pi)\left(\alpha_{1}, \alpha_{2}, \alpha_{3}\right)\right)(1 \cdot 1+\mathbf{e} \cdot \mathbf{e})=-(1 \cdot 1+\mathbf{e} \cdot \mathbf{e}),
\end{gathered}
$$

we obtain

$$
\left(V_{\Lambda}\right)_{K_{[\mathfrak{a}]}} \cap W_{\Lambda_{1}^{\prime}}=\mathbf{C}(1 \cdot 1-\mathbf{e} \cdot \mathbf{e}),
$$

and thus $\Lambda=y_{1}+y_{2}+y_{3}+y_{4} \in D\left(K, K_{[\mathfrak{a}]}\right)$, which has eigenvalue 30 of $-\mathcal{C}_{L}$ with the multiplicity 1 . Therefore,

$$
\begin{aligned}
n\left(L^{30}\right) & =\operatorname{dim}_{\mathbf{C}} V_{(6,1,1,1,0,0)}+\operatorname{dim}_{\mathbf{C}} V_{(-6,1,1,1,0,0)}+\operatorname{dim}_{\mathbf{C}} V_{(0,1,1,1,0,0)} \\
& =120+120+210=450 \\
& =\operatorname{dim} S O(32)-\operatorname{dim} U(1) \cdot \operatorname{Spin}(10)=n_{k l}\left(L^{30}\right) .
\end{aligned}
$$

Then we conclude that

THEOREM 5.6. The Gauss image

$$
L^{30}=(U(1) \cdot \operatorname{Spin}(10)) /\left(S^{1} \cdot \operatorname{Spin}(6) \cdot \mathbf{Z}_{4}\right) \subset Q_{30}(\mathbf{C})
$$

is strictly Hamiltonian stable.

Combining with the results on Hamiltonian stabilities of Gauss images of all homogeneous isoparametric hypersurfaces, we obtain our main theorem.

\section{REFERENCES}

[1] K. Abe And I. YoкотA, Realization of spaces $E_{6} /(U(1) \operatorname{Spin}(10)), E_{7} /\left(U(1) E_{6}\right), E_{8} /\left(U(1) E_{7}\right)$ and their volumes, Tokyo J. Math. 20 (1997), no. 1, 73-86.

[2] A. AmARZAYA AND Y. OHNitA, Hamiltonian stability of certain minimal Lagrangian submanifolds in complex projective spaces, Tohoku Math. J.(2) 55 (2003), 583-610.

[ 3 ] T. AsoH, Compact transformation groups on $\mathbf{Z}_{2}$-cohomology spheres with orbits of codimension 1, Hiroshima Math. J. 11 (1981), 571-616.

[ 4 ] T. AsOH, Supplement to "Compact transformation groups on $\mathbf{Z}_{2}$-cohomology spheres with orbits of codimension 1", Hiroshima Math. J. 13 (1983), 647-652.

[ 5 ] N. Bourbaki, Elements de mathematique. Fasc. XXXIV. Groupes et algebres de Lie, Actualites Scientifiques et Industrielles, No. 1337, Hermann, Paris, 1968.

[6] C. Chavalley and R. D. Schafer, The exceptional simple Lie algebras $F_{4}$ and $E_{6}$, Proc. Nat. Acad. Sci. U.S.A. 36 (1950), 137-141.

[ 7 ] H. Freudenthal, Oktaven, Ausnahmegruppen and Oktavengeometrie, Utrecht, 1951, reprinted in Geom. Dedicata 19 (1985), 7-63.

[ 8 ] M. Goto and F. D. Grosshans, Semisimple Lie algebras, Lecture Notes in Pure and Applied Mathematics, Vol. 38. Marcel Dekker, Inc., New York-Basel, 1978.

[9] W.-Y. Hsiang And H. B. Lawson, JR., Minimal submanifolds of low cohomogeneity, J. Differential Geometry 5 (1971), 1-38.

[10] M. IsE, Bounded symmetric domains of exceptional type, J. Fac. Sci. Univ. Tokyo Sect. IA Math. 23 (1976), 75-105. 
[11] J. Lepowsky, Multiplicity formulas for certain semisimple Lie groups, Bull. Amer. Math. Soc. 77 (1971), 601-605.

[12] H. MA AND Y. OHNITA, On Lagrangian submanifolds in complex hyperquadrics and isoparametric hypersurfaces in spheres, Math. Z. 261 (2009), 749-785.

[13] H. MA AND Y. OHNitA, Differential geometry of Lagrangian submanifolds and Hamiltonian variational problems (Ed. E. Loubeau and S. Montaldo), Proceedings of the Conference in honor of J. C. Wood's sixtieth birthday, 115-134, Contemp. Math. 542, Amer. Math. Soc., Providence, RI, 2011.

[14] H. MA AND Y. OHNitA, Hamiltonian stability of the Gauss images of homogeneous isoparametric hypersurfaces I, J. Differential Geom. 97 (2014), 275-348.

[15] W. G. McKay And J. PAtera, Tables of dimensions, indices, and branching rules for representations of simple Lie algebras, Lecture Notes in Pure and Applied Mathematics, Vol. 69. Marcel Dekker, Inc., New York-Basel, 1981.

[16] H. F. MÜnZnER, Isoparametrische Hyperfläche in Sphären, Math. Ann. 251 (1980), 57-71.

[17] H. F. MÜNZNER, Isoparametrische Hyperfläche in Sphären, II, Math. Ann. 256 (1981), 215-232.

[18] Y. G. OH, Second variation and stabilities of minimal Lagrangian submanifolds in Kähler manifolds, Invent. Math. 101 (1990), 501-519.

[19] Y. OHNITA, Geometry of Lagrangian submanifolds and isoparametric hypersurfaces, Proceedings of The Fourteenth International Workshop on Differential Geometry and Related Fields [Volume 14], 43-67, ed. by Y.-J. Suh, National Institute for Mathematical Sciences, The Korean Mathematical Society and Grassmann Research Group, Natl. Inst. Math. Sci. (NIMS), Taejŏn, 2010.

[20] H. OzEKI AND M. TAKEUCHI, On some types of isoparametric hypersurfaces in spheres I, Tohoku Math. J.(2) 27 (1975), 515-559.

[21] H. OZEKI AND M. TAKEUCHI, On some types of isoparametric hypersurfaces in spheres II, Tohoku Math. J.(2) 28 (1976), 7-55.

[22] R. TAKAGI AND T. TAKAHASHI, On the principal curvatures of homogeneous hypersurfaces in a unit sphere, Differential Geometry, in honor of K. Yano, 469-481, Kinokuniya, Tokyo, 1972.

[23] M. TAKEUCHI, Modern theory of spherical functions, Translations of Mathematical Monographs, AMS, 1993.

[24] C. TsukAmoto, Spectra of Laplace-Beltrami operators on $S O(n+2) / S O(2) \times S O(n)$ and $S p(n+1) / S p(1) \times$ Sp (n), Osaka J. Math. 18 (1981), 407-426.

[25] F. UCHIDA, An orthogonal transformation group of (8k-1)-sphere, J. Differential Geom. 15 (1980), 569-574.

[26] S. Yamaguchi, Spectra of flag manifolds, Mem. Fac. Sci. Kyushu Univ. Ser. A 33 (1979), 95-112.

[27] I. YокотA, Exceptional Lie Groups, arXiv:0902.0431v1[mathDG], 2009.

DePartment of Mathematical SCIEnCES

TSINGHUA UNIVERSITY

BEIJING 100084

P.R. CHINA

E-mail address: hma@math.tsinghua.edu.cn

\author{
AdVAnCED Mathematical Institute \& \\ DEPARTMENT OF MATHEMATICS \\ OSAKA CITY UNIVERSITY \\ 3-3-138 SUGIMOTO, SUMIYOSHI-KU \\ OSAKA 558-8585 \\ JAPAN
}

E-mail address: ohnita@sci.osaka-cu.ac.jp 